TRANSACTIONS OF THE

AMERICAN MATHEMATICAL SOCIETY

Volume 362, Number 12, December 2010, Pages 6551-6590

S 0002-9947(2010)05096-2

Article electronically published on July 13, 2010

\title{
COHOMOLOGY AND SUPPORT VARIETIES FOR LIE SUPERALGEBRAS
}

\author{
BRIAN D. BOE, JONATHAN R. KUJAWA, AND DANIEL K. NAKANO
}

\begin{abstract}
Unlike Lie algebras, the finite dimensional complex representations of a simple Lie superalgebra are usually not semisimple. As a consequence, despite over thirty years of study, these remain mysterious objects. In this paper we introduce a new tool: the notion of cohomological support varieties for the finite dimensional supermodules for a classical Lie superalgebra $\mathfrak{g}=\mathfrak{g}_{\overline{0}} \oplus \mathfrak{g}_{\overline{1}}$ which are completely reducible over $\mathfrak{g}_{\overline{0}}$. They allow us to provide a new, functorial description of the previously combinatorial notions of defect and atypicality. We also introduce the detecting subalgebra of $\mathfrak{g}$. Its role is analogous to the defect subgroup in the theory of finite groups in positive characteristic. Using invariant theory we prove that there are close connections between the cohomology and support varieties of $\mathfrak{g}$ and the detecting subalgebra.
\end{abstract}

\section{INTRODUCTION}

1.1. The blocks of Category $\mathcal{O}$ (or relative Category $\mathcal{O}_{S}$ ) for complex semisimple Lie algebras are well-known examples of highest weight categories, as defined in [CPS], with finitely many simple modules. These facts imply that the projective resolutions for modules in these categories have finite length, so the cohomology (or extensions) can be non-zero in only finitely many degrees.

On the other hand, one can consider the category $\mathcal{F}$ of finite dimensional supermodules for a classical Lie superalgebra $\mathfrak{g}($ e.g. $\mathfrak{g}=\mathfrak{g l}(m \mid n))$ which are completely reducible over $\mathfrak{g}_{\overline{0}}$. For $\mathfrak{g l}(m \mid n)$ this was shown to be a highest weight category by Brundan [Bru1] and recently also for osp $(2 \mid 2 n)$ by Cheng, Wang, and Zhang CWZ. However, these categories and most blocks of these categories contain infinitely many simple modules, and projective resolutions often have infinite length. Cohomology can be non-zero in infinitely many degrees and in fact can have polynomial rate of growth.

A similar scenario has been successfully handled for modular representations of finite groups and restricted Lie algebras by the use of support varieties. Remarkably, an analogous theory holds for $\mathfrak{g}$-supermodules in $\mathcal{F}$. In this paper we develop this algebro-geometric approach and explore the connections it provides between

Received by the editors April 13, 2009.

2010 Mathematics Subject Classification. Primary 17B56, 17B10; Secondary 13A50.

The research of the first author was partially supported by NSA grant H98230-04-1-0103.

The research of the second author was partially supported by NSF grants DMS-0402916 and DMS-0734226.

The research of the third author was partially supported by NSF grants DMS-0400548 and DMS-0654169.

(C)2010 American Mathematical Society Reverts to public domain 28 years from publication 
representation theory, cohomology, and combinatorics of classical Lie superalgebras and the category $\mathcal{F}$.

1.2. Our approach incorporates many previously known computations related to relative cohomology for Lie superalgebras by Brundan Bru1, Fuks and Leites [FL, and Gruson Gru1, Gru2 . It should be noted that it is important to use relative cohomology for our purposes rather than ordinary Lie superalgebra cohomology. The latter is usually non-zero in only finitely many degrees (cf. [Fuk, Gru1]) and thus will not capture much information about the representation theory of $\mathfrak{g}$.

Let us set some notation. Let $\mathfrak{g}=\mathfrak{g}_{\overline{0}} \oplus \mathfrak{g}_{\overline{1}}$ be a simple classical Lie superalgebra over the complex numbers as classified by Kac [Kac1]. A description of the simple classical Lie superalgebras can be found in Sections 8.1 8.7. Denote by $G_{\overline{0}}$ the connected reductive algebraic group such that $\operatorname{Lie}\left(G_{\overline{0}}\right)=\mathfrak{g}_{\overline{0}}$.

Perhaps the most striking outcome of our investigation is that, under certain mild assumptions, $\mathfrak{g}$ contains Lie subsuperalgebras which "detect" the cohomology of $\mathfrak{g}$. In Section 4 we construct these Lie subsuperalgebras of $\mathfrak{g}$ and show that they arise naturally from results in the invariant theory of reductive groups due to Luna and Richardson [LR, and Dadok and Kac [DK]. In particular, if $R=\mathrm{H}^{\bullet}\left(\mathfrak{g}, \mathfrak{g}_{0} ; \mathbb{C}\right)$ is the cohomology for $\mathfrak{g}$ relative to $\mathfrak{g}_{\overline{0}}$, then there exists a Lie subsuperalgebra $\mathfrak{e}=\mathfrak{e}_{\overline{0}} \oplus \mathfrak{e}_{\overline{1}}$ such that

$$
R \cong S^{\bullet}\left(\mathfrak{g}_{\overline{1}}^{*}\right)^{G_{\overline{0}}} \cong S^{\bullet}\left(\mathfrak{e}_{\overline{1}}^{*}\right)^{W} \cong \mathrm{H}^{\bullet}\left(\mathfrak{e}, \mathfrak{e}_{\overline{0}} ; \mathbb{C}\right)^{W},
$$

where $W$ is a finite pseudoreflection group. From this isomorphism one sees that $R$ is a finitely generated algebra and in fact a polynomial algebra. To see an example of the subsuperalgebra $\mathfrak{e}$, we refer the reader to Sections 8.10 8.11 where the type A case is considered in some detail.

The subsuperalgebra $\mathfrak{e}$ can be viewed as an analogue of a Sylow subgroup with the above theorem looking very much like a theorem involving transfer for finite groups. The reader may also notice that the aforementioned isomorphism is reminiscent of Borel's calculation of the rational cohomology for the classifying space of a compact Lie group.

In recent years there has been active and ongoing interest in the development and use of support variety theories in various contexts (cf. Balmer Bal, ErdmannHolloway [EH], Friedlander-Pevtsova [FP2, and Snashall-Solberg [SS]). By using the finite generation of $R$ we develop such a theory for $\mathfrak{g}$-supermodules in the category $\mathcal{F}$. Given such a $\mathfrak{g}$-supermodule $M$ one can consider the support varieties $\mathcal{V}_{\left(\mathfrak{g}, \mathfrak{g}_{\overline{0}}\right)}(M)$ and $\mathcal{V}_{\left(\mathfrak{e}, \mathfrak{e}_{\overline{0}}\right)}(M)$. As (1.2.1) suggests, there is a close relationship between these two varieties. More precisely, in Theorem 6.2 we prove that $\mathcal{V}_{\left(\mathfrak{g}, \mathfrak{g}_{\overline{0}}\right)}(M)$ is "generically" isomorphic to $\mathcal{V}_{\left(\mathfrak{e}, \mathfrak{e}_{\overline{0}}\right)}(M) / W$.

Motivated by this relationship we more closely study the variety $\mathcal{V}_{\left(\mathfrak{e}, \mathfrak{e}_{\overline{0}}\right)}(M)$ in Section 6. We prove that $\mathcal{V}_{\left(\mathfrak{e}, \mathfrak{e}_{\overline{0}}\right)}(M)$ can be identified via a "rank variety" description as a certain affine subvariety of $\mathfrak{e}_{\overline{1}}$. This concrete description allows us to verify that the operator $\mathcal{V}_{\left(\mathfrak{e}, \mathfrak{e}_{\mathfrak{0}}\right)}(-)$ satisfies many of the desirable properties of a support variety theory. In particular, it detects projectivity in the sense of Dade's Lemma for finite groups and has the tensor product property (see Proposition 6.3 and Theorem [6.6). Furthermore, one sees that the representation theory for the superalgebra $\mathfrak{e}$ over $\mathbb{C}$ has features similar to that of modular representations over 
fields of characteristic two (cf. Corollary 6.5). One interesting facet of our definition of rank varieties is that it involves restricting supermodules to copies of the Lie superalgebra $\mathfrak{q}(1)$ rather than cyclic shifted subgroups.

In using these geometric and cohomological methods we also aim to obtain a deeper understanding of the combinatorics of the finite dimensional representations of the Lie superalgebra $\mathfrak{g}$. Let $\mathfrak{g}$ be a simple classical Lie superalgebra with a non-degenerate invariant supersymmetric even bilinear form. Kac and Wakimoto KW use the form to give a combinatorial definition of the (numerical) defect of $\mathfrak{g}$. The subsuperalgebras we introduce can be viewed as "defect subalgebras" of the principal block whose odd dimension coincides with the defect described above. We also provide a cohomological interpretation of the defect of a Lie superalgebra which suggests a natural extension of the notion to Lie superalgebras without such a bilinear form.

Kac and Wakimoto also use the form on $\mathfrak{g}$ to define the atypicality of a block and of a simple $\mathfrak{g}$-supermodule. Atypicality is a combinatorial invariant which roughly measures the intricacy of the block and its representations. For instance, the characters of the typical (i.e. atypicality zero) simple supermodules for the basic classical Lie superalgebras were obtained early on by Kac Kac2. On the other hand, much effort has gone into studying atypical simple supermodules, with most investigations being handled on a case-by-case basis (e.g. MVdJ, Ser2, Ser3, VdJZ, VdJ]). Character formulas in terms of Kazhdan-Lusztig polynomials were obtained in type A by Serganova Ser1 and, with different methods, by Brundan Bru1. Analogous results for type Q were obtained by Penkov and Serganova PS] and, again by different methods, by Brundan [Bru2. In [CWZ the type C case was handled using the techniques introduced by Brundan. Despite all these efforts, the atypical blocks remain mysterious. Part of the goal of this paper is to introduce new techniques which shed insight on atypicality and which may be applied in the less studied cases; for example, the Cartan type Lie superalgebras and type P (cf. Ser4, Ser2]). In the last section of the paper we discuss how both the defect and atypicality are related to our support variety constructions. We formulate an intriguing conjecture regarding this relationship which suggests a functorial description of atypicality. We also discuss how our conjecture is closely related to a conjecture of Kac and Wakimoto.

We remark that the approach taken here differs considerably from that of Duflo and Serganova DS. Certainly our varieties are not equal to theirs, as our rank varieties contain elements whose bracket with themselves is non-zero. As far as we know, the associated varieties they construct do not have a cohomological interpretation. It would be of great interest to describe the relationship, if any, between these two theories.

1.3. A number of further developments which heavily depend on the foundational results of this paper have arisen since this manuscript first appeared as a preprint. In BKN] the authors prove that in the case of $\mathfrak{g}=\mathfrak{g l}(m \mid n)$ the support varieties considered here are compatible with certain translation and restriction functors, introduced by Serganova [Ser5], between blocks of category $\mathcal{F}$. As a consequence the support varieties of the finite dimensional simple $\mathfrak{g l}(m \mid n)$-supermodules are explicitly described as unions of affine spaces which are conjugate under the action of a reflection group. One outcome of this calculation is that Conjecture 7.2 holds true for $\mathfrak{g l}(m \mid n)$. Namely, the dimension of the support variety of a finite dimensional 
simple $\mathfrak{g l}(m \mid n)$-supermodule is equal to the supermodule's degree of atypicality. Thus support varieties provide a natural functorial and geometric interpretation of the combinatorial notion of atypicality.

More generally, if $\mathfrak{g}$ is a Type I Lie superalgebra (i.e. $\mathfrak{g}$ admits a $\mathbb{Z}$-grading concentrated in degrees $-1,0,1$ which is compatible with the $\mathbb{Z}_{2}$-grading), we show in BKN that the category $\mathcal{F}$ is a highest weight category in the sense of Cline, Parshall, and Scott CPS. Furthermore, in this case the support varieties of the Kac supermodules (the standard objects in these highest weight categories) are zero. On the other hand, the support varieties of Kac supermodules (i.e. the universal highest weight supermodules in $\mathcal{F}$ ) are commonly non-zero when $\mathfrak{g}$ is not of Type $\mathrm{I}$. In this way support varieties perceive that $\mathfrak{g}$ is of Type I, an important distinction first made by Kac Kac1.

As an application of the theory introduced in this paper, in [BKN] the authors use the detecting superalgebras defined here to prove that the dimension of a supermodule $M$ is divisible by a power of 2 , where the power is directly related to the codimension of the support variety of $M$. In many ways this can be viewed as a characteristic zero super analogue of the Kac-Weisfeiler Conjecture [KWe]. Recall that this conjecture asserts that for Lie algebras in characteristic $p>0$ the dimension of an indecomposable module is divisible by a power of $p$ which depends only on the block containing the module. This was originally proven by Premet Pre] using support varieties.

Recently Wang and Zhao WZ1, WZ2 formulated a characteristic $p$ super analogue of the Kac-Weisfeiler Conjecture in which the dimension of a supermodule is divisible both by a power of 2 as in BKN and a power of $p$ as in $\mathrm{KWe}$. They prove their conjecture for the basic classical Lie superalgebras and blocks of $\mathfrak{q}(n)$ with nilpotent character using finite $W$-superalgebras. It would be interesting to develop a characteristic $p$ analogue of the support varieties considered here. One would expect that such a theory would naturally relate to the conjecture of Wang and Zhao.

Finally, let us mention the results of Bagci, Kujawa, and Nakano in BaKN. They show that the results of the present paper have a natural extension to the Cartan type Lie superalgebra $W(n)$. An essential difference is that one should consider the pair $\left(W(n), W(n)_{0}\right)$ rather than $\left(W(n), W(n)_{\overline{0}}\right)$, where $W(n)_{0}$ is the degree 0 part of $W(n)$ in its canonical $\mathbb{Z}$-grading. They show the cohomology ring is again a polynomial ring and $W(n)$ admits a natural detecting subalgebra. Using results of Serganova Ser4 they are able to compute the support varieties of the finite dimensional simple supermodules and again the dimensions coincide with the combinatorial notion of atypicality introduced in Ser5]. These results were further extended to the Cartan type Lie superalgebra $S(n)$ by Bagci Bag.

In a general result which includes the classical Lie superalgebras considered in this paper, the authors of $[\mathrm{BaKN}]$ prove that the realizability result for detecting subalgebras proven here (Theorem 6.7) can be lifted to the support varieties for $\mathfrak{g}$. Namely, if $X$ is an arbitrary conical subvariety of the maximal ideal spectrum of the cohomology ring of $\mathfrak{g}$, then there exists a finite dimensional $\mathfrak{g}$-supermodule whose support variety is precisely $X$. In this way one sees that the geometry which arises from support varieties is as complex as could be hoped for. In light of this realization theorem, they conjecture that one should be able to use support varieties to prove that most blocks of the category $\mathcal{F}$ have wild representation type, using 
arguments similar to those used by Farnsteiner [Far] in the setting of finite group schemes in positive characteristic.

The authors would like to acknowledge David Benson, William Graham, Markus Hunziker, Gerald Schwarz, and Robert Varley for sharing their insights during various stages of this project.

\section{Lie SUPERALGEBRAS, REPRESENTATIONS, AND COHOMOLOGY}

2.1. Lie superalgebras and their representations. Throughout we work with the complex numbers $\mathbb{C}$ as the ground field. Recall that a superspace is a $\mathbb{Z}_{2^{-}}$ graded vector space, and given a superspace $V$ and a homogeneous vector $v \in V$, we write $\bar{v} \in \mathbb{Z}_{2}$ for the parity (or degree) of $v$. Elements of $V_{\overline{0}}$ (resp. $V_{\overline{1}}$ ) are called even (resp. odd). Note that if $M$ and $M^{\prime}$ are two superspaces, then the space $\operatorname{Hom}_{\mathbb{C}}\left(M, M^{\prime}\right)$ is naturally $\mathbb{Z}_{2}$-graded by declaring $f \in \operatorname{Hom}_{\mathbb{C}}\left(M, M^{\prime}\right)_{r}\left(r \in \mathbb{Z}_{2}\right)$ if $f\left(M_{s}\right) \subseteq M_{s+r}^{\prime}$ for all $s \in \mathbb{Z}_{2}$.

A superalgebra is a $\mathbb{Z}_{2}$-graded, unital, associative algebra $A=A_{\overline{0}} \oplus A_{\overline{1}}$ which satisfies $A_{r} A_{s} \subseteq A_{r+s}$ for all $r, s \in \mathbb{Z}_{2}$. A Lie superalgebra is a superspace $\mathfrak{g}=\mathfrak{g}_{\overline{0}} \oplus \mathfrak{g}_{\overline{1}}$ with a bracket operation $[]:, \mathfrak{g} \otimes \mathfrak{g} \rightarrow \mathfrak{g}$ which preserves the $\mathbb{Z}_{2}$-grading and satisfies graded versions of the usual Lie bracket axioms. In particular, we note that $\mathfrak{g}_{\overline{0}}$ is a Lie algebra under the bracket obtained by restricting the bracket of $\mathfrak{g}$. If $\mathfrak{g}$ is a Lie superalgebra, then one has a universal enveloping superalgebra $U(\mathfrak{g})$ which is $\mathbb{Z}_{2}$-graded and satisfies a PBW type theorem. See, for example, [Kac1] for details and further background on Lie superalgebras.

We call a finite dimensional Lie superalgebra classical if there is a connected reductive algebraic group $G_{\overline{0}}$ such that $\operatorname{Lie}\left(G_{\overline{0}}\right)=\mathfrak{g}_{\overline{0}}$, and an action of $G_{\overline{0}}$ on $\mathfrak{g}_{\overline{1}}$ which differentiates to the adjoint action of $\mathfrak{g}_{\overline{0}}$ on $\mathfrak{g}_{\overline{1}}$. In particular, if $\mathfrak{g}$ is classical, then $\mathfrak{g}_{\overline{0}}$ is a reductive Lie algebra and $\mathfrak{g}_{\overline{1}}$ is semisimple as a $\mathfrak{g}_{\overline{0}}$-module. Note that we do not assume that $\mathfrak{g}$ is simple. A basic classical Lie superalgebra is a classical Lie superalgebra with a non-degenerate invariant supersymmetric even bilinear form. The simple (basic) classical Lie superalgebras were classified by Kac Kac1].

Given a Lie superalgebra, $\mathfrak{g}$, let us describe the category of $\mathfrak{g}$-supermodules. The objects are all left $U(\mathfrak{g})$-modules which are $\mathbb{Z}_{2}$-graded; that is, superspaces $M$ satisfying $U(\mathfrak{g})_{r} M_{s} \subseteq M_{r+s}$ for all $r, s \in \mathbb{Z}_{2}$. If $M$ is a $\mathfrak{g}$-supermodule, then by definition $N \subseteq M$ is a subsupermodule if it is a supermodule which inherits its grading from $M$ in the sense that $M_{r} \cap N=N_{r}$ for $r \in \mathbb{Z}_{2}$. Given $\mathfrak{g}$-supermodules $M$ and $N$ one can use the antipode and coproduct of $U(\mathfrak{g})$ to define a $\mathfrak{g}$-supermodule structure on the contragradient dual $M^{*}$ and the tensor product $M \otimes N$.

A morphism of $U(\mathfrak{g})$-supermodules is an element of $\operatorname{Hom}_{\mathbb{C}}\left(M, M^{\prime}\right)$ satisfying $f(x m)=(-1)^{\bar{f}} \bar{x} x f(m)$ for all $m \in M$ and all $x \in U(\mathfrak{g})$. Note that this definition makes sense as stated only for homogeneous elements; it should be interpreted via linearity in the general case. We emphasize that we allow all morphisms and not just graded (i.e. even) morphisms. However, note that $\operatorname{Hom}_{U(\mathfrak{g})}\left(M, M^{\prime}\right)$ inherits a $\mathbb{Z}_{2}$-grading as a subspace of $\operatorname{Hom}_{\mathbb{C}}\left(M, M^{\prime}\right)$.

The category of $\mathfrak{g}$-supermodules is not an abelian category. However, the underlying even category, consisting of the same objects but only the even morphisms, is an abelian category. This, along with the parity change functor, $\Pi$, which simply interchanges the $\mathbb{Z}_{2}$-grading of a supermodule, allows one to make use of the tools of homological algebra. 
As a special case of the above discussion, we always view a Lie algebra (e.g. the even part of a Lie superalgebra) as a Lie superalgebra concentrated in degree $\overline{0}$.

There are two categories of $\mathfrak{g}$-supermodules which are natural to consider. First, recall that one says a supermodule $M$ is finitely semisimple if it is isomorphic to a direct sum of finite dimensional simple subsupermodules. If $\mathfrak{g}$ is a Lie superalgebra and $\mathfrak{t}$ is a Lie subsuperalgebra, then let $\mathcal{C}=\mathcal{C}_{(\mathfrak{g}, \mathfrak{t})}$ denote the full subcategory of the category of all $\mathfrak{g}$-supermodules obtained by taking as objects all $\mathfrak{g}$-supermodules which are finitely semisimple as $\mathbf{t}$-supermodules.

We let $\mathcal{F}=\mathcal{F}_{(\mathfrak{g}, \mathfrak{t})}$ denote the full subcategory of $\mathcal{C}$ obtained by taking the objects to be all finite dimensional supermodules in $\mathcal{C}$. Observe that in the case when $\mathfrak{t}=\mathfrak{g}_{0}$ is semisimple as a Lie algebra, $\mathcal{F}$ is simply the category of finite dimensional $\mathfrak{g}$-supermodules.

As discussed in [Kum, 3.1.6], the category $\mathcal{C}$ is closed under arbitrary direct sums, quotients, and finite tensor products.

2.2. Relative cohomology. In this subsection we outline the basic definitions and results for relative cohomology for Lie superalgebras. Relative cohomology for Lie algebras was first defined by Hochschild [Hoc, and the super case is considered in Fuks [Fuk]. The main theme is that, once one accounts for the $\mathbb{Z}_{2}$-grading, results from the purely even case hold here as well. For the sake of brevity we omit proofs when they are straightforward generalizations of the classical arguments. We refer the reader to Kum, Appendix D] for the details.

Let $R$ be a superalgebra and $S$ a subsuperalgebra. In particular, we assume $S_{r}=R_{r} \cap S$ for $r \in \mathbb{Z}_{2}$. Let

$$
\cdots \rightarrow M_{i-1} \stackrel{f_{i-1}}{\longrightarrow} M_{i} \stackrel{f_{i}}{\longrightarrow} M_{i+1} \rightarrow \cdots
$$

be a sequence of $R$-supermodules and even $R$-supermodule homomorphisms. We say this sequence is $(R, S)$-exact if it is exact as a sequence of $R$-supermodules and if, when viewed as a sequence of $S$-supermodules, $\operatorname{Ker} f_{i}$ is a direct summand of $M_{i}$ for all $i$. Note that our assumption that $f_{i}$ is even implies that $\operatorname{Ker} f_{i}$ is a subsupermodule of $M_{i}$ and that the splitting $M_{i}=\operatorname{Ker} f_{i} \oplus N_{i}$ is as $S$-supermodules.

An $R$-supermodule $P$ is $(R, S)$-projective if given any $(R, S)$-exact sequence

$$
0 \rightarrow M_{1} \stackrel{f}{\rightarrow} M_{2} \stackrel{g}{\rightarrow} M_{3} \rightarrow 0
$$

and $R$-supermodule homomorphism $h: P \rightarrow M_{3}$ there is an $R$-supermodule map $\tilde{h}: P \rightarrow M_{2}$ satisfying $g \circ \tilde{h}=h$.

In particular, if $P$ is a projective $R$-supermodule, then it is automatically $(R, S)$ projective. Also, since $g$ is assumed to be even, if $h$ is homogeneous, then one can choose $\tilde{h}$ to be homogeneous of the same degree as $h$. More generally, if we write $h=h_{\overline{0}}+h_{\overline{1}}$ where $h_{r} \in \operatorname{Hom}_{R}\left(P, M_{3}\right)_{r}(r=\overline{0}, \overline{1})$, then we can lift each $h_{r}$ and $\widetilde{h_{\overline{0}}}+\widetilde{h_{\overline{1}}}$ is a lift of $h$.

An $(R, S)$-projective resolution of an $R$-supermodule $M$ is an $(R, S)$-exact sequence

$$
\stackrel{\delta_{2}}{\longrightarrow} P_{1} \stackrel{\delta_{1}}{\longrightarrow} P_{0} \stackrel{\delta_{0}}{\longrightarrow} M \rightarrow 0,
$$

where each $P_{i}$ is an $(R, S)$-projective supermodule. We remind the reader that implicit in the definition is the fact that the maps $\delta_{i}$ are all assumed to be even.

The following lemma is proven just as in Kum. 
Lemma 2.1. Let $R$ be a superalgebra and $S$ be a subsuperalgebra of $R$.

(a) If $M$ is any $S$-supermodule, then $R \otimes_{S} M$ is an $(R, S)$-projective $R$-supermodule. The $\mathbb{Z}_{2}$-grading on $R \otimes_{S} M$ is given in the usual way by

$$
\left(R \otimes_{S} M\right)_{i}=\bigoplus_{\substack{k, l \in \mathbb{Z}_{2} \\ k+l=i}} R_{k} \otimes_{S} M_{l} .
$$

(b) Any $R$-supermodule $M$ admits an $(R, S)$-projective resolution. Namely,

$$
\cdots \stackrel{\delta_{2}}{\longrightarrow} R \otimes_{S} \operatorname{Ker} \delta_{0} \stackrel{\delta_{1}}{\longrightarrow} R \otimes_{S} M \stackrel{\delta_{0}}{\longrightarrow} M \rightarrow 0 .
$$

Here $\delta_{i}$ is the "multiplication" map $R \otimes_{S} N \rightarrow N$ given by $r \otimes n \mapsto r n$ for any $R$-supermodule $N$.

Note that since the multiplication map is even, its kernel is an $R$-subsupermodule of the domain, and we can recursively define the above sequence as indicated. Given an $R$-supermodule $M$ with $(R, S)$-projective resolution $P_{\bullet} \rightarrow M$, apply the functor $\operatorname{Hom}_{R}(-, N)$ and set

$$
\operatorname{Ext}_{(R, S)}^{i}(M, N)=\mathrm{H}^{i}\left(\operatorname{Hom}_{R}\left(P_{\bullet}, N\right)\right) .
$$

One can show that $\operatorname{Ext}_{(R, S)}^{\bullet}(M, N)$ can also be obtained using the dually defined $(R, S)$-injective resolutions and that it is functorial in both arguments and is well defined. See $\mathrm{Kum}$ for details. It is of interest to track the $\mathbb{Z}_{2}$-grading. As we remarked earlier, $\operatorname{Hom}_{R}\left(P_{i}, N\right)$ is naturally $\mathbb{Z}_{2}$-graded and since $\delta_{i}$ was assumed to be even, the induced homomorphism $\operatorname{Hom}_{R}\left(P_{i-1}, N\right) \rightarrow \operatorname{Hom}_{R}\left(P_{i}, N\right)$ is also even. Consequently, $\operatorname{Ext}_{(R, S)}^{i}(M, N)$ inherits a $\mathbb{Z}_{2}$-grading.

2.3. Relative cohomology for Lie superalgebras. Let $\mathfrak{g}$ be a Lie superalgebra and let $\mathfrak{t} \subseteq \mathfrak{g}$ be a Lie subsuperalgebra. In this section we define (relative) Lie superalgebra cohomology for the pair $(\mathfrak{g}, \mathfrak{t})$ and prove that it coincides with the relative cohomology defined in the previous section for the enveloping superalgebras of the pair.

First let us recall the definition of Lie superalgebra cohomology. Let $M$ be a $\mathfrak{g}$-supermodule. For $p \geq 0$, set

$$
C^{p}(\mathfrak{g}, M)=\operatorname{Hom}_{\mathbb{C}}\left(\bigwedge_{s}^{p}(\mathfrak{g}), M\right),
$$

where $\bigwedge_{s}^{p}(\mathfrak{g})$ is the super wedge product. That is, $\bigwedge_{s}^{p}(\mathfrak{g})$ is the $p$-fold tensor product of $\mathfrak{g}$ modulo the $\mathfrak{g}$-subsupermodule generated by elements of the form

$$
x_{1} \otimes \cdots \otimes x_{k} \otimes x_{k+1} \otimes \cdots \otimes x_{p}+(-1)^{\bar{x}_{k} \bar{x}_{k+1}} x_{1} \otimes \cdots \otimes x_{k+1} \otimes x_{k} \otimes \cdots \otimes x_{p},
$$

for homogeneous $x_{1}, \ldots, x_{p} \in \mathfrak{g}$. Thus $x_{k}, x_{k+1}$ skew commute unless both are odd, in which case they commute.

Let

$$
d^{p}: C^{p}(\mathfrak{g}, M) \rightarrow C^{p+1}(\mathfrak{g}, M)
$$

be given by

$$
\begin{aligned}
& d^{p}(\phi)\left(x_{1} \wedge \cdots \wedge x_{p+1}\right) \\
= & \sum_{i<j}(-1)^{\sigma_{i, j}\left(x_{1}, \ldots, x_{p}\right)} \phi\left(\left[x_{i}, x_{j}\right] \wedge x_{1} \wedge \cdots \wedge \hat{x}_{i} \wedge \cdots \wedge \hat{x}_{j} \wedge \cdots \wedge x_{p+1}\right) \\
& +\sum_{i}(-1)^{\gamma_{i}\left(x_{1}, \ldots, x_{p}, \phi\right)} x_{i} \phi\left(x_{1} \wedge \cdots \wedge \hat{x}_{i} \wedge \cdots \wedge x_{p+1}\right),
\end{aligned}
$$


where $x_{1}, \ldots, x_{p+1}$ and $\phi$ are assumed to be homogeneous, and

$$
\begin{aligned}
\sigma_{i, j}\left(x_{1}, \ldots, x_{p}\right) & :=i+j+\bar{x}_{i}\left(\bar{x}_{1}+\cdots+\bar{x}_{i-1}\right)+\bar{x}_{j}\left(\bar{x}_{1}+\cdots+\bar{x}_{j-1}+\bar{x}_{i}\right), \\
\gamma_{i}\left(x_{1}, \ldots, x_{p}, \phi\right) & :=i+1+\bar{x}_{i}\left(\bar{x}_{1}+\cdots+\bar{x}_{i-1}+\bar{\phi}\right) .
\end{aligned}
$$

Then we define

$$
\mathrm{H}^{p}(\mathfrak{g}, M)=\operatorname{Ker} d^{p} / \operatorname{Im} d^{p-1} .
$$

Now consider the relative version of the above construction. Let $\mathfrak{g}, \mathfrak{t}$, and $M$ be as above. Define

$$
C^{p}(\mathfrak{g}, \mathfrak{t} ; M)=\operatorname{Hom}_{\mathfrak{t}}\left(\bigwedge_{s}^{p}(\mathfrak{g} / \mathfrak{t}), M\right) .
$$

Then the map $d^{p}$ descends to give a map $d^{p}: C^{p}(\mathfrak{g}, \mathfrak{t} ; M) \rightarrow C^{p+1}(\mathfrak{g}, \mathfrak{t} ; M)$ and we define

$$
\mathrm{H}^{p}(\mathfrak{g}, \mathfrak{t} ; M)=\operatorname{Ker} d^{p} / \operatorname{Im} d^{p-1} .
$$

As discussed earlier Hom spaces are naturally $\mathbb{Z}_{2}$-graded so the cochains have a $\mathbb{Z}_{2}$-grading. Note that the map $d^{p}$ preserves this grading, and so the cohomology inherits a $\mathbb{Z}_{2}$-grading. Thus cohomology is $\mathbb{Z} \times \mathbb{Z}_{2}$-graded — by the cohomological degree and the $\mathbb{Z}_{2}$-grading, respectively.

Let us now relate the two cohomologies we have introduced. The proof given in Kum can be adapted to the super setting to yield the following result.

Lemma 2.2. Let $\mathfrak{g}$ be a Lie superalgebra, $\mathfrak{t}$ a Lie subsuperalgebra, and $M, N \mathfrak{g}$ supermodules. Assume that $\mathfrak{g}$ is finitely semisimple as a $\mathfrak{t}$-supermodule under the adjoint action. Then

$$
\operatorname{Ext}_{(\mathcal{U}(\mathfrak{g}), \mathcal{U}(\mathfrak{t}))}(M, N) \cong \operatorname{Ext}_{(\mathcal{U}(\mathfrak{g}), \mathcal{U}(\mathfrak{t}))}\left(\mathbb{C}, M^{*} \otimes N\right) \cong \mathrm{H}^{\bullet}\left(\mathfrak{g}, \mathfrak{t} ; M^{*} \otimes N\right) .
$$

2.4. Categorical cohomology. In this subsection we interpret relative cohomology in terms of cohomology in certain categories. Our approach is inspired by that of [GJ]. Fix a Lie superalgebra $\mathfrak{g}$ and a Lie subsuperalgebra $\mathfrak{t}$. Let $\mathcal{C}=\mathcal{C}_{(\mathfrak{g}, \mathfrak{t})}$ and $\mathcal{F}=\mathcal{F}_{(\mathfrak{g}, \mathfrak{t})}$ be the categories introduced in Section 2.1.

Proposition 2.3. Assume $\mathfrak{g}$ is finitely semisimple as a $\mathfrak{t}$-supermodule under the adjoint action. Let $L$ be a finite dimensional simple $\mathfrak{t}$-supermodule. Let

$$
\widehat{L}=\mathcal{U}(\mathfrak{g}) \otimes_{\mathcal{U}(\mathfrak{t})} L .
$$

Then $\widehat{L}$ is $(\mathcal{U}(\mathfrak{g}), \mathcal{U}(\mathfrak{t}))$-projective and a projective supermodule in the category $\mathcal{C}_{(\mathfrak{g}, \mathfrak{t})}$.

Proof. Note that in the definition of $\widehat{L}$ we view $\mathcal{U}(\mathfrak{g})$ as a right $\mathcal{U}(\mathfrak{t})$-supermodule via right multiplication, and $\widehat{L}$ as a left $\mathcal{U}(\mathfrak{g})$-supermodule via left multiplication. By Lemma $2.1 \widehat{L}$ is $(\mathcal{U}(\mathfrak{g}), \mathcal{U}(\mathfrak{t}))$-projective.

Now since $\mathcal{C}$ is closed under tensor products, arbitrary direct sums, and quotients, $\mathcal{U}(\mathfrak{g})$ is a finitely semisimple $\mathfrak{t}$-supermodule under the adjoint action. Consequently, $\mathcal{U}(\mathfrak{g}) \otimes_{\mathbb{C}} L$ is a finitely semisimple $\mathfrak{t}$-supermodule. It remains to observe that the induced module $\widehat{L}$ can be obtained as a quotient of $\mathcal{U}(\mathfrak{g}) \otimes_{\mathbb{C}} L$ (cf. the proof of $\underline{\mathrm{Kum}}$, Corollary 3.1.8]). Consequently, $\widehat{L}$ is finitely semisimple and hence is an object in $\mathcal{C}_{(\mathfrak{g}, \mathfrak{t})}$. Finally, one can verify that $\widehat{L}$ is a projective object in $\mathcal{C}_{(\mathfrak{g}, \mathfrak{t})}$ using Frobenius reciprocity.

Corollary 2.4. Assume $\mathfrak{g}$ is finitely semisimple as a $\mathfrak{t}$-supermodule under the adjoint action. Let $\mathcal{D}$ be a full subcategory of $\mathcal{C}_{(\mathfrak{g}, \mathfrak{t})}$ such that whenever $L$ is a finite dimensional simple $\mathfrak{t}$-supermodule which appears as a composition factor of some 
object in $\mathcal{D}$, then $\widehat{L}$ is an object in $\mathcal{D}$. Furthermore, assume $\mathcal{D}$ is closed under direct sums. Then $\mathcal{D}$ has enough projectives and for any $M, N$ which are objects in $\mathcal{D}$ we have

as superspaces.

$$
\operatorname{Ext}_{\mathcal{D}}^{p}(M, N) \cong \operatorname{Ext}_{(\mathcal{U}(\mathfrak{g}), \mathcal{U}(\mathfrak{t}))}^{p}(M, N)
$$

Proof. Let $M$ be an object in $\mathcal{D}$ and write $M$ as the direct sum of finite dimensional simple $\mathrm{t}$-supermodules:

$$
M=\bigoplus_{j \in I} L_{j}
$$

Then by Frobenius reciprocity one sees that $P:=\bigoplus_{j \in I} \widehat{L}_{j}$ maps onto $M$. By the previous proposition $P$ is projective in $\mathcal{D}$, and so $\mathcal{D}$ has enough projectives. Therefore, we also see that any object in $\mathcal{D}$ has a resolution by supermodules which are both projective in $\mathcal{D}$ and $(\mathcal{U}(\mathfrak{g}), \mathcal{U}(\mathfrak{t}))$-projective. In order to compute cohomology in both categories one applies $\operatorname{Hom}_{U(\mathfrak{g})}(-, N)$ to this resolution; thus the equivalence of Exts follows.

2.5. Cohomology for the pair $\left(\mathfrak{g}, \mathfrak{g}_{\overline{0}}\right)$. For the remainder of the article we will make the following assumptions. We will always assume $\mathfrak{g}$ is a classical Lie superalgebra. A $\mathfrak{g}$-supermodule will always be assumed to be an object in the category $\mathcal{C}=\mathcal{C}_{\left(\mathfrak{g}, \mathfrak{g}_{\overline{0}}\right)}$, and a finite dimensional $\mathfrak{g}$-supermodule will always mean an object in the category $\mathcal{F}=\mathcal{F}_{\left(\mathfrak{g}, \mathfrak{g}_{\overline{0}}\right)}$.

By the PBW theorem for Lie superalgebras (cf. Kac1, 1.1.3]), if $N$ is a finite dimensional $\mathfrak{g}_{0}$-supermodule, then

$$
U(\mathfrak{g}) \otimes_{U\left(\mathfrak{g}_{\overline{0}}\right)} N
$$

is again finite dimensional. Therefore the conditions of Corollary 2.4 are satisfied for the pair $\left(\mathfrak{g}, \mathfrak{g}_{\overline{0}}\right)$ where the category $\mathcal{D}$ is $\mathcal{F}$. Combining that result with Lemma 2.2 yields the following theorem.

Theorem 2.5. Let $M$ and $N$ be finite dimensional $\mathfrak{g}$-supermodules. Then for all $p \geq 0$ we have

$$
\operatorname{Ext}_{\mathcal{F}}^{p}(M, N) \cong \mathrm{H}^{p}\left(\mathfrak{g}, \mathfrak{g}_{\overline{0}} ; M^{*} \otimes N\right) .
$$

In particular, we can apply the above result when $M=N=\mathbb{C}$ to obtain the cohomology ring for $\mathfrak{g}$ in the category $\mathcal{F}$. Before doing so, let us make several observations which will simplify the calculation.

We first note that the differential $d^{p}$ in (2.3.1) is identically zero. Namely, since the bracket preserves the $\mathbb{Z}_{2}$-grading we have $\left[\mathfrak{g}_{\overline{1}}, \mathfrak{g}_{\overline{1}}\right] \subseteq \mathfrak{g}_{\overline{0}}$. From this observation one concludes that in the first sum of (2.3.1) each $\left[x_{i}, x_{j}\right]$ is always zero in the quotient $\mathfrak{g} / \mathfrak{g}_{\overline{0}}$, and hence these terms are identically zero. The terms in the second sum of (2.3.1) are all zero since here $M$ is the trivial supermodule. Consequently, the cohomology is simply the cochains themselves. Second, we observe that $\mathfrak{g} / \mathfrak{g}_{\overline{0}} \cong$ $\mathfrak{g}_{\overline{1}}$ as a $\mathfrak{g}_{\overline{0}}$-supermodule. Taken together, these observations imply

$$
\operatorname{Ext}_{\mathcal{F}}^{p}(\mathbb{C}, \mathbb{C}) \cong \operatorname{Hom}_{\mathfrak{g}_{\overline{0}}}\left(\bigwedge_{s}^{p}\left(\mathfrak{g}_{\overline{1}}\right), \mathbb{C}\right) \text {. }
$$

However, one can simplify further with the following observation. Since $\mathfrak{g}_{\overline{1}}$ is purely odd the elements of $\bigwedge_{s}^{p}\left(\mathfrak{g}_{\overline{1}}\right)$ commute without sign, and so this super wedge product can be viewed as a classical symmetric product. Let $G_{\overline{0}}$ denote the connected reductive group with Lie algebra $\mathfrak{g}_{\overline{0}}$. We then obtain the following theorem. 
Theorem 2.6. Let $\mathfrak{g}$ be a classical Lie superalgebra and let $\mathcal{F}$ be the category of finite dimensional $\mathfrak{g}$-supermodules. Then

$$
\operatorname{Ext}_{\mathcal{F}}^{\bullet}(\mathbb{C}, \mathbb{C}) \cong \mathrm{H}^{\bullet}\left(\mathfrak{g}, \mathfrak{g}_{\overline{0}} ; \mathbb{C}\right) \cong S\left(\mathfrak{g}_{\overline{1}}^{*}\right)^{\mathfrak{g}_{\overline{0}}}=S\left(\mathfrak{g}_{\overline{1}}^{*}\right)^{G_{\overline{0}}}
$$

The last equality is a well known result in characteristic zero Lie theory (cf. TY, 24.3.3]). As a matter of notation we write $S\left(\mathfrak{g}_{1}^{*}\right)$ for $S^{\bullet}\left(\mathfrak{g}_{1}^{*}\right)$.

Note that one can check by the definition that this isomorphism is one of superalgebras; that is, the map respects multiplication. Since $S\left(\mathfrak{g}_{1}^{*}\right)$ is generated by elements of degree $(1, \overline{1})$ in the $\mathbb{Z} \times \mathbb{Z}_{2}$-grading, both it and the cohomology ring will consist of elements whose $\mathbb{Z}_{2}$ degree is the reduction modulo 2 of the cohomological degree. In particular, the cohomology ring is always a commutative ring in the ungraded sense. Because of this and as the $\mathbb{Z}_{2}$-grading will not generally play an important role in what follows, we will leave it implicit unless otherwise necessary.

Recall that if $M$ is a finite dimensional $\mathfrak{g}$-supermodule, then $\operatorname{Ext}_{\mathcal{F}}(\mathbb{C}, M)=$ $\mathrm{H}^{\bullet}\left(\mathfrak{g}, \mathfrak{g}_{0} ; M\right)$ is an $\operatorname{Ext}_{\mathcal{F}}^{\bullet}(\mathbb{C}, \mathbb{C})=\mathrm{H}^{\bullet}\left(\mathfrak{g}, \mathfrak{g}_{0} ; \mathbb{C}\right)$-module via the Yoneda product (cf. Ben, 5.7]).

Theorem 2.7. Let $\mathfrak{g}$ be a classical Lie superalgebra. Let $M$ be a finite dimensional $\mathfrak{g}$-supermodule. The superalgebra $\mathrm{H}^{\bullet}\left(\mathfrak{g}, \mathfrak{g}_{0} ; \mathbb{C}\right)$ is finitely generated as a ring. Furthermore, $\mathrm{H}^{\bullet}\left(\mathfrak{g}, \mathfrak{g}_{0} ; M\right)$ is finitely generated as an $\mathrm{H}^{\bullet}\left(\mathfrak{g}, \mathfrak{g}_{0} ; \mathbb{C}\right)$-module.

Proof. The fact that $\mathrm{H}^{\bullet}\left(\mathfrak{g}, \mathfrak{g}_{0} ; \mathbb{C}\right)$ is finitely generated is immediate from Theorem 2.6 and the classic invariant theory result of Hilbert [PV, Theorem 3.6]. Now consider the second statement of the theorem. Clearly,

$$
\bigoplus_{p \geq 0} \operatorname{Hom}_{\mathbb{C}}\left(\bigwedge_{s}^{p}\left(\mathfrak{g}_{\overline{1}}\right), M\right) \cong \bigoplus_{p \geq 0}\left(\bigwedge_{s}^{p}\left(\mathfrak{g}_{1}^{*}\right) \otimes M\right) \cong S\left(\mathfrak{g}_{1}^{*}\right) \otimes M
$$

is finitely generated as an $S\left(\mathfrak{g}_{1}^{*}\right)$-module. Applying [PV, 3.25], we see that

$$
\left(\bigoplus_{p \geq 0} \operatorname{Hom}_{\mathbb{C}}\left(\bigwedge_{s}^{p}\left(\mathfrak{g}_{\overline{1}}\right), M\right)\right)^{\mathfrak{g}_{\overline{0}}}=\bigoplus_{p \geq 0} \operatorname{Hom}_{\mathfrak{g}_{\overline{0}}}\left(\bigwedge_{s}^{p}\left(\mathfrak{g}_{\overline{1}}\right), M\right)
$$

is finitely generated as an $S\left(\mathfrak{g}_{1}^{*}\right)^{G_{\overline{0}}}=\mathrm{H}^{\bullet}\left(\mathfrak{g}, \mathfrak{g}_{\overline{0}} ; \mathbb{C}\right)$-module. That is, the cochain complex $C^{\bullet}\left(\mathfrak{g}, \mathfrak{g}_{\overline{0}} ; M\right)$ used in Section 2.3 to define $\mathrm{H}^{\bullet}\left(\mathfrak{g}, \mathfrak{g}_{\overline{0}} ; M\right)$ is finitely generated as a module over $\mathrm{H}^{\bullet}\left(\mathfrak{g}, \mathfrak{g}_{\overline{0}} ; \mathbb{C}\right)$.

The finite generation of $C^{\bullet}\left(\mathfrak{g}, \mathfrak{g}_{0} ; M\right)$ implies that $\mathrm{H}^{\bullet}\left(\mathfrak{g}, \mathfrak{g}_{0} ; M\right)$ is finitely generated as follows. Given $r \in \mathrm{H}^{p}\left(\mathfrak{g}, \mathfrak{g}_{\overline{0}} ; \mathbb{C}\right)$ and $x \in C^{\bullet}\left(\mathfrak{g}, \mathfrak{g}_{\overline{0}} ; M\right)$, one has $d(r x)=$ $d(r) x+(-1)^{p} r d(x)=(-1)^{p} r d(x)$, where $p$ is the cohomological degree of $r$ (cf. the proof of [Gru1, Theorem 5]). The second equality follows from the fact that the differentials for $\mathrm{H}^{\bullet}\left(\mathfrak{g}, \mathfrak{g}_{0} ; \mathbb{C}\right)$ are identically zero. Therefore, $d: C^{\bullet}\left(\mathfrak{g}, \mathfrak{g}_{\overline{0}} ; M\right) \rightarrow$ $C^{\bullet}\left(\mathfrak{g}, \mathfrak{g}_{0} ; M\right)$ is a graded $\mathrm{H}^{\bullet}\left(\mathfrak{g}, \mathfrak{g}_{\overline{0}} ; \mathbb{C}\right)$-module homomorphism. Since $\mathrm{H}^{\bullet}\left(\mathfrak{g}, \mathfrak{g}_{\overline{0}} ; \mathbb{C}\right)$ is finitely generated, any subquotient of a finitely generated graded module is finitely generated. This implies the final statement of the theorem.

\section{INVARIANT THEORY}

3.1. In this section we develop some of the invariant theory needed to study cohomology in the category $\mathcal{F}$. More specifically, we will study the action of $G_{\overline{0}}$ on $\mathfrak{g}_{\overline{1}}$. When the action is either stable (see Section 3.2) or polar (see Section 3.3) there are general results about rings of invariants which will be central to the approach taken here. These results will be used in Section 4 to provide more structural information 
pertaining to the relative cohomology groups for these Lie superalgebras. By doing a case-by-case analysis one can determine which simple classical Lie superalgebras and related algebras admit stable and/or polar actions. This data is presented in Table 5 of the Appendix.

We view $\mathfrak{g}_{\overline{1}}$ as an affine variety with the Zariski topology. It is isomorphic to the affine space $\mathbb{A}^{\operatorname{dim} \mathfrak{g}_{\overline{1}}}$ and so is smooth and irreducible. The group $G_{\overline{0}}$ acts via the adjoint action. Given $g \in G_{\overline{0}}$ and $x \in \mathfrak{g}_{\overline{1}}$, we write $g . x$ for this action. Given $x \in \mathfrak{g}_{\overline{1}}$, let $G_{\overline{0}, x}$ denote the stabilizer subgroup $G_{\overline{0}, x}=\left\{g \in G_{\overline{0}} \mid g \cdot x=x\right\}$ and let $G_{\overline{0}} \cdot x=\left\{g \cdot x \mid g \in G_{\overline{0}}\right\}$.

3.2. Stable actions. Let us recall the following definitions and results from invariant theory:

(a) A point $x \in \mathfrak{g}_{\overline{1}}$ is regular if $G_{\overline{0}} \cdot x$ has the maximum possible dimension. Equivalently, $x$ is regular if $G_{\overline{0}, x}$ has minimal dimension. By [PV, Sec. 1.4] there is a dense open subset of $\mathfrak{g}_{\overline{1}}$ consisting of regular points.

(b) A point $x \in \mathfrak{g}_{\overline{1}}$ is semisimple if the orbit $G_{\overline{0}} \cdot x$ is closed in $\mathfrak{g}_{\overline{1}}$.

(c) The action of $G_{\overline{0}}$ on $\mathfrak{g}_{\overline{1}}$ is called stable if there is an open dense subset of $\mathfrak{g}_{\overline{1}}$ consisting of semisimple points. By a theorem of Popov Pop the action of $G_{\overline{0}}$ on $\mathfrak{g}_{\overline{1}}$ will be stable if and only if $\mathfrak{g}_{\overline{1}}$ has regular semisimple points.

(d) If there is an open subset of $\mathfrak{g}_{\overline{1}}$ such that the stabilizer subgroups of any two points in this set are conjugate subgroups of $G_{\overline{0}}$, then the stabilizer of such a point is called a stabilizer in general position. By a theorem of Richardson [PV, Theorem 7.2], such an open set exists in $\mathfrak{g}_{\overline{1}}$.

(e) If the action of $G_{\overline{0}}$ on $\mathfrak{g}_{\overline{1}}$ is stable, then one has that $\mathfrak{g}_{\overline{1}}$ contains an open dense set of regular semisimple elements whose stabilizers are in general position. We call such points generic.

For short we say $\mathfrak{g}$ is stable if the action of $G_{\overline{0}}$ on $\mathfrak{g}_{\overline{1}}$ is stable. Assume $\mathfrak{g}$ is stable. Fix a generic element $x_{0} \in \mathfrak{g}_{\overline{1}}$ and set

$$
H=G_{\overline{0}, x_{0}} .
$$

Note that if $\pi: \mathfrak{g}_{\overline{1}} \rightarrow \mathfrak{g}_{\overline{1}} / G_{\overline{0}}$ is the canonical projection, then $\pi\left(x_{0}\right) \in \mathfrak{g}_{\overline{1}} / G_{\overline{0}}$ is principal in the sense of $\mathrm{LR}$. Therefore we can utilize the generalization of the Chevalley Restriction Theorem given by Luna and Richardson in [LR]. Set

$$
\mathfrak{f}_{\overline{1}}=\mathfrak{g}_{\overline{1}}^{H}=\left\{z \in \mathfrak{g}_{\overline{1}} \mid h . z=z \text { for all } h \in H\right\}
$$

and set

$$
N=N_{G_{\overline{0}}}(H)=\left\{g \in G_{\overline{0}} \mid g H g^{-1}=H\right\}=\left\{g \in G_{\overline{0}} \mid g \cdot \mathfrak{f}_{\overline{1}}=\mathfrak{f}_{\overline{1}}\right\} .
$$

Since $x_{0}$ is semisimple one knows that $H$ is reductive and hence, by LR, Lemma 1.1], so is $N$.

We are now prepared to state the following key theorem.

Theorem 3.1. Let $\mathfrak{g}$ be a classical Lie superalgebra which is stable. Let $M$ be a finite dimensional $G_{\overline{0}}$-module.

(a) The restriction homomorphism $S\left(\mathfrak{g}_{1}^{*}\right) \rightarrow S\left(\mathfrak{f}_{\overline{1}}^{*}\right)$ induces an isomorphism

$$
\text { res : } S\left(\mathfrak{g}_{\overline{1}}^{*}\right)^{G_{\overline{0}}} \rightarrow S\left(\mathfrak{f}_{\overline{1}}^{*}\right)^{N} \text {. }
$$

(b) The set $G_{\overline{0}} \cdot f_{\overline{1}}$ is dense in $\mathfrak{g}_{\overline{1}}$. 
(c) The map induced by restriction

$$
\rho: \operatorname{Hom}_{G_{\overline{0}}}\left(S^{n}\left(\mathfrak{g}_{\overline{1}}\right), M\right) \rightarrow \operatorname{Hom}_{N}\left(S^{n}\left(\mathfrak{f}_{\overline{1}}\right), M\right)
$$

is injective for any $n \geq 0$.

Proof. (a)-(b) Part (a) is a direct application of [LR, Corollary 4.4], and (b) follows by $\mathrm{PV}$, Section 7.1].

(c) It is convenient to dualize the situation. That is, we instead show

$$
\rho: \operatorname{Hom}_{G_{\overline{0}}}\left(M, S^{n}\left(\mathfrak{g}_{\overline{1}}^{*}\right)\right) \rightarrow \operatorname{Hom}_{N}\left(M, S^{n}\left(\mathfrak{f}_{\overline{1}}^{*}\right)\right)
$$

is injective for any $G_{\overline{0}}$-module $M$. This is equivalent to the claimed statement.

Let $\varphi \in \operatorname{Hom}_{G_{\overline{0}}}\left(M, S^{n}\left(\mathfrak{g}_{1}^{*}\right)\right)$ such that $\rho(\varphi)=0$. That is, for any $m \in M$, we have $\rho(\varphi)(m) \in S^{n}\left(\mathfrak{f}_{\overline{1}}^{*}\right)$ is zero as a homogenous degree $n$ polynomial on $\mathfrak{f}_{\overline{1}}$. In other words, $\varphi(m)(y)=0$ for all $m \in M$ and all $y \in \mathfrak{f}_{\overline{1}}$. Since $\varphi$ is a $G_{\overline{0}}$-homomorphism, for any fixed $m \in M$ we have

$$
\varphi(m)(g \cdot y)=\varphi\left(g^{-1} \cdot m\right)(y)=0
$$

for all $g \in G_{\overline{0}}$ and $y \in \mathfrak{f}_{\overline{1}}$. That is, $\varphi(m)$ is identically zero on $G_{\overline{0}} \cdot \mathfrak{f}_{\overline{1}}$. However, by part (b) this implies that $\varphi(m)=0$. Since $m \in M$ was arbitrary, this implies that $\varphi$ itself is identically zero.

3.3. Polar representations. We now recall the notion of polar representations introduced by Dadok and Kac [DK]. Let $K$ be a reductive algebraic group acting on a vector space $V$. Let $v \in V$ be a semisimple element. Let

$$
\mathfrak{e}_{v}=\{x \in V \mid \mathfrak{k} . x \subseteq \mathfrak{k} . v\},
$$

where $\mathfrak{k}$ is the Lie algebra of $K$. In general one has $\operatorname{dim} \mathfrak{e}_{v} \leq \operatorname{dim} S\left(V^{*}\right)^{K}$. By definition the action of $K$ on $V$ is polar if for some semisimple $v \in V$ we have $\operatorname{dim} \mathfrak{e}_{v}=\operatorname{dim} S\left(V^{*}\right)^{K}$. In this case $\mathfrak{e}_{v}$ is called a Cartan subspace. For brevity we say $\mathfrak{g}$ is polar when the action of $G_{\overline{0}}$ on $\mathfrak{g}_{\overline{1}}$ is polar. We write $\mathfrak{e}_{\overline{1}}$ for our fixed choice of a Cartan subspace.

In the case when the action of $G_{\overline{0}}$ on $\mathfrak{g}_{\overline{1}}$ is both stable and polar, one can further assume

$$
x_{0} \in \mathfrak{e}_{x_{0}}=\mathfrak{e}_{\overline{1}} \subseteq \mathfrak{f}_{\overline{1}},
$$

where $x_{0}$ and $\mathfrak{f}_{\overline{1}}$ are as in Section 3.2 (cf. [DK, p. 514]). When the action is stable and polar, the Cartan subspace is unique up to conjugation by $G_{\overline{0}}$ by $[\mathrm{DK}$, Theorem $2.3]$.

Let us set the following notation. Given a subspace $V \subseteq \mathfrak{g}_{\overline{1}}$, let

$$
\begin{aligned}
& \operatorname{Norm}_{G_{\overline{0}}}(V)=\left\{g \in G_{\overline{0}} \mid g \cdot V=V\right\}, \\
& \operatorname{Stab}_{G_{\overline{0}}}(V)=\left\{g \in G_{\overline{0}} \mid g \cdot v=v \text { for all } v \in V\right\} .
\end{aligned}
$$

One has the following theorem.

Theorem 3.2. Assume $\mathfrak{g}$ is a classical Lie superalgebra which is polar. Let $\mathfrak{e}_{\overline{1}}$ be a Cartan subspace of $\mathfrak{g}_{\overline{1}}$. Let $M$ be a $G_{\overline{0}}$-module. Then

(a) Restriction of functions then defines an isomorphism

$$
S\left(\mathfrak{g}_{\overline{1}}^{*}\right)^{G_{\overline{0}}} \cong S\left(\mathfrak{e}_{\overline{1}}^{*}\right)^{W},
$$

where

$$
W:=\operatorname{Norm}_{G_{\overline{0}}}\left(\mathfrak{e}_{\overline{1}}\right) / \operatorname{Stab}_{G_{\overline{0}}}\left(\mathfrak{e}_{\overline{1}}\right) .
$$

Furthermore, $W$ is necessarily a finite pseudoreflection group. 
(b) If $\mathfrak{g}$ is also stable, then $G_{\overline{0}} \cdot \mathfrak{e}_{\overline{1}}$ is dense in $\mathfrak{g}_{\overline{1}}$.

(c) If $\mathfrak{g}$ is also stable, then the map induced by restriction

$$
\rho: \operatorname{Hom}_{G_{\overline{0}}}\left(S^{n}\left(\mathfrak{g}_{\overline{1}}\right), M\right) \rightarrow \operatorname{Hom}_{N^{\prime}}\left(S^{n}\left(\mathfrak{e}_{\overline{1}}\right), M\right)
$$

is injective for any $n \geq 0$, where $N^{\prime}:=\operatorname{Norm}_{G_{\overline{0}}}\left(\mathfrak{e}_{\overline{1}}\right)$.

Proof. (a) This follows from [DK, Theorems 2.9-2.10]. (b) Since we assume the action of $G_{\overline{0}}$ on $\mathfrak{g}_{\overline{1}}$ is stable, there is an open dense subset of $\mathfrak{g}_{\overline{1}}$ consisting of semisimple elements. By [DK, Proposition 2.2] every semisimple element of $\mathfrak{g}_{\overline{1}}$ is conjugate to some element of $\mathfrak{e}_{\overline{1}}$. The result then follows. (c) This follows by the same reasoning as in the proof of Theorem 3.1 (c).

Assume $\mathfrak{g}$ is both stable and polar. Recall that the discriminant of the action of $W$ on $\mathfrak{e}_{\overline{1}}$ is an element $D \in S\left(\mathfrak{e}_{\overline{1}}^{*}\right)^{W}$ where, for any $y \in \mathfrak{e}_{\overline{1}}, D(y) \neq 0$ if and only if $W_{y}$ is trivial. To be concrete, one can take $D$ to be a sufficiently high power of the Jacobian, $J$ (recalling that $J \in S\left(\mathfrak{e}_{1}^{*}\right)$ is a skew invariant which satisfies $J(y) \neq 0$ if and only if $W_{y}$ is trivial). The Jacobian for the polar Lie superalgebras considered in Sections 8.18.7 can be found in Table 5. By doing a case-by-case check one can verify that for the simple classical Lie superalgebras which are stable and polar, $y \in \mathfrak{g}_{\overline{1}}$ is generic if and only if $D(y) \neq 0$. Therefore, in these cases the set of generic points of $\mathfrak{g}_{\overline{1}}$ is precisely the principal open set defined by $D$.

3.4. We now recall a generalization of a theorem of Kostant and Rallis as presented in [GW, 12.4.6].

Theorem 3.3. Let $K$ be a connected, reductive, linear algebraic group and let $V$ be a regular representation of $K$, and assume there is a subspace $\mathfrak{a} \subseteq V$ such that the following holds:

(1) Restriction of functions defines an isomorphism of $S\left(V^{*}\right)^{K}$ onto a subalgebra $\mathcal{R}$ of $S\left(\mathfrak{a}^{*}\right)$.

(2) The subalgebra $\mathcal{R}$ is generated by algebraically independent homogeneous elements $u_{1}, \ldots, u_{r}$ with $r=\operatorname{dim} \mathfrak{a}$. Furthermore, there exists a graded subspace $\mathcal{A}$ of $S\left(\mathfrak{a}^{*}\right)$ such that the multiplication map $\mathcal{A} \otimes \mathcal{R} \rightarrow S\left(\mathfrak{a}^{*}\right)$ is a linear isomorphism.

(3) There exists $h \in \mathfrak{a}$ such that $|K . h \cap \mathfrak{a}| \geq \operatorname{dim} \mathcal{A}$.

(4) Let $h$ be as above, and set

$$
\begin{aligned}
& \qquad \mathcal{X}_{h}=\left\{v \in V \mid f(v)=f(h) \text { for all } f \in S\left(V^{*}\right)^{K}\right\} . \\
& \text { If } v \in \mathcal{X}_{h} \text {, then }
\end{aligned}
$$

$$
\operatorname{dim} K \cdot v=\operatorname{dim} V-\operatorname{dim} \mathfrak{a} .
$$

Assuming the above hypotheses hold, there exists a graded $K$-submodule $\mathcal{H} \subseteq S\left(V^{*}\right)$ such that multiplication provides an isomorphism of graded right $S\left(V^{*}\right)^{K}$-modules,

$$
\mathcal{H} \otimes S\left(V^{*}\right)^{K} \rightarrow S\left(V^{*}\right) .
$$

Furthermore,

$$
\mathcal{H} \cong \operatorname{ind}_{K_{h}}^{K} \mathbb{C}
$$

as $K$-modules, where $K_{h}$ is the stabilizer of $h$ in $K$. 
3.5. Throughout this subsection we assume $\mathfrak{g}$ is stable and polar. We first apply Theorem 3.3 to the action of $G_{\overline{0}}$ on $\mathfrak{g}_{\overline{1}}$. Set $K=G_{\overline{0}}$ and $V=\mathfrak{g}_{\overline{1}}$. Let $\mathfrak{a}=\mathfrak{e}_{\overline{1}}$, the Cartan subspace fixed in Section 3.3 .

Theorem 3.4. Let $x_{0} \in \mathfrak{g}_{\overline{1}}$ be our fixed generic point and let $H=G_{\overline{0}, x_{0}}$. Then there exists a graded $G_{\overline{0}}$-module $\mathcal{H} \subseteq S\left(\mathfrak{g}_{\overline{1}}^{*}\right)$ such that the multiplication map

$$
\mathcal{H} \otimes S\left(\mathfrak{g}_{\overline{1}}^{*}\right)^{G_{\overline{0}}} \rightarrow S\left(\mathfrak{g}_{\overline{1}}^{*}\right)
$$

gives an isomorphism of graded right $S\left(\mathfrak{g}_{\overline{1}}^{*}\right)^{G_{\overline{0}}}$-modules. Furthermore, $\mathcal{H} \cong \operatorname{ind}_{H}^{G_{\overline{0}}} \mathbb{C}$ as a $G_{\overline{0}}$-module.

Proof. We simply need to verify that the conditions of Theorem 3.3 apply.

(1) By Theorem 3.2 restriction provides an isomorphism

$$
S\left(\mathfrak{g}_{\overline{1}}^{*}\right)^{G_{\overline{0}}} \rightarrow S\left(\mathfrak{e}_{\overline{1}}^{*}\right)^{W},
$$

where $W$ is as given in the theorem.

(2) Since $W$ is a finite pseudoreflection group, it follows immediately that $S\left(\mathfrak{g}_{1}^{*}\right)^{G_{\overline{0}}}$ is generated by $\operatorname{dim} \mathfrak{e}_{\overline{1}}$ algebraically independent generators. There exists a graded subspace $\mathcal{A} \subseteq S\left(\mathfrak{e}_{1}^{*}\right)$ such that multiplication $\mathcal{A} \otimes S\left(\mathfrak{e}_{1}^{*}\right)^{W} \rightarrow$ $S\left(\mathfrak{e}_{1}^{*}\right)$ provides a graded linear isomorphism. Furthermore, we have $\operatorname{dim} \mathcal{A}=$ $|W|$. See, for example, GW, Theorem 12.4.7] for a summary of these well known results.

(3) Let $h=x_{0} \in \mathfrak{e}_{\overline{1}}$. Since $\mathfrak{e}_{\overline{1}} \subseteq \mathfrak{f}_{\overline{1}}=\mathfrak{g}_{\overline{1}}^{H}$ we have that $H \subseteq \operatorname{Stab}_{G_{\overline{0}}}\left(\mathfrak{e}_{\overline{1}}\right)$, and so we have

$$
\left|G_{\overline{0}} \cdot x_{0} \cap \mathfrak{e}_{\overline{1}}\right| \geq\left|\operatorname{Norm}_{G_{\overline{0}}}\left(\mathfrak{e}_{\overline{1}}\right) \cdot x_{0}\right| \geq\left|W \cdot x_{0}\right|=|W|=\operatorname{dim} \mathcal{A} .
$$

(4) If $\pi: \mathfrak{g}_{\overline{1}} \rightarrow \mathfrak{g}_{\overline{1}} / G_{\overline{0}}$ is the canonical quotient map, then by definition (see [PV], Sec. 4.4])

$$
\mathcal{X}_{x_{0}}=\pi^{-1}\left(\pi\left(x_{0}\right)\right)
$$

However, since $x_{0}$ is semisimple and regular, the fiber $\pi^{-1}\left(\pi\left(x_{0}\right)\right)$ is precisely $G_{\overline{0}} \cdot x_{0}$. Therefore it suffices to check (3.4.1) for $x_{0}$. Now since $x_{0}$ is generic, by [Sha, Theorem $7(\mathrm{ii})]$ one has that

$$
\begin{aligned}
\operatorname{dim} G_{\overline{0}} \cdot x_{0} & =\operatorname{dim} \mathfrak{g}_{\overline{1}}-\operatorname{dim} \mathfrak{g}_{\overline{1}} / G_{\overline{0}} \\
& =\operatorname{dim} \mathfrak{g}_{\overline{1}}-\operatorname{dim} \mathfrak{e}_{\overline{1}},
\end{aligned}
$$

where the last equality follows from the fact that the Krull dimension of $S\left(\mathfrak{g}_{\overline{1}}^{*}\right)^{G_{\overline{0}}}$ equals the dimension of $\mathfrak{e}_{\overline{1}}$.

Now consider the group $N$ acting on $\mathfrak{f}_{\overline{1}}$. Note that in the following theorem there is the additional assumption that the stabilizer of a generic point is connected. However, we verified by direct calculation that this condition is satisfied for all the Lie superalgebras considered in Sections 8.1 8.7 which are stable and polar. The stabilizer $H$ in these cases can be found in Table 4 .

Theorem 3.5. Let $x_{0} \in \mathfrak{g}_{\overline{1}}$ be our fixed generic point and let $H=G_{\overline{0}, x_{0}}$. Assume $H$ is connected. Then there exists a graded $N$-module $\mathcal{H} \subseteq S\left(\mathfrak{f}_{\overline{1}}^{*}\right)$ such that the multiplication map

$$
\mathcal{H} \otimes S\left(\mathfrak{f}_{1}^{*}\right)^{N} \rightarrow S\left(\mathfrak{f}_{\frac{1}{1}}^{*}\right)
$$

gives an isomorphism of graded right $S\left(\mathfrak{f}_{\frac{1}{1}}^{*}\right)^{N}$-modules. Furthermore, $\mathcal{H} \cong \operatorname{ind}_{H}^{N} \mathbb{C}$ as $N$-modules. 
Proof. Since $N$ is in general not connected, one cannot apply Theorem 3.3 directly. Write $N^{0}$ for the connected component of the identity of $N$, and instead apply Theorem 3.3 to $N^{0}$ acting on $\mathfrak{f}_{\overline{1}}$. This action is stable. Indeed, one sees that $x_{0} \in \mathfrak{f}_{\overline{1}}$ is a generic element as follows. Since the $N^{0}$-stabilizer of any point of $\mathfrak{f}_{\overline{1}}$ contains $H, x_{0}$ is regular. To show that $x_{0}$ is semisimple, first note by Luna's criterion $\mathrm{PV}$, Theorem 6.17] that N. $x_{0}$ is closed in $\mathfrak{f}_{\overline{1}}$. Using that $H \subseteq N^{0}$, one sees that $N . x_{0}$ is the disjoint union of a finite number of $N^{0}$-orbits, each of the same dimension as $N^{0} . x_{0}$. Therefore each of them, including $N^{0} . x_{0}$, is closed.

The action of $N^{0}$ on $\mathfrak{f}_{\overline{1}}$ is also polar with Cartan subspace $\mathfrak{e}_{\overline{1}}$. This can be seen as follows. Since the action of $G_{\overline{0}}$ on $\mathfrak{g}_{\overline{1}}$ is both stable and polar, DK, Corollary 2.5] implies $\mathfrak{g}_{\overline{1}}=\mathfrak{e}_{\overline{1}} \oplus \mathfrak{g}_{\overline{0}} \cdot x_{0}$. Also, by assumption, $\mathfrak{e}_{\overline{1}} \subseteq \mathfrak{f}_{\overline{1}}$. Taken together this implies $\mathfrak{e}_{\overline{1}} \oplus \operatorname{Lie}\left(N^{0}\right) \cdot x_{0} \subseteq \mathfrak{f}_{\overline{1}}$. However, one has

$$
\operatorname{dim} \mathfrak{e}_{\overline{1}}=\operatorname{dim} \mathfrak{g}_{\overline{1}} / G_{\overline{0}}=\operatorname{dim} \mathfrak{f}_{\overline{1}} / N=\operatorname{dim} \mathfrak{f}_{\overline{1}} / N^{0}=\operatorname{dim} \mathfrak{f}_{\overline{1}}-\operatorname{dim} \operatorname{Lie}\left(N^{0}\right) \cdot x_{0} .
$$

The first equality holds because $\mathfrak{e}_{\overline{1}}$ is a Cartan subspace for the polar action of $G_{\overline{0}}$ on $\mathfrak{g}_{\overline{1}}$, the second equality follows from Theorem 3.1(a), the third equality holds because $N / N^{0}$ is a finite group, and the last equality holds because $x_{0}$ is a generic point for the action of $N^{0}$ on $\mathfrak{f}_{\overline{1}}$. Therefore, by dimension counting, $\mathfrak{e}_{\overline{1}} \oplus \operatorname{Lie}\left(N^{0}\right) \cdot x_{0}=\mathfrak{f}_{\overline{1}}$. This, along with the fact that $\mathfrak{e}_{\overline{1}}$ is a Cartan subspace for the action of $G_{\overline{0}}$ on $\mathfrak{g}_{\overline{1}}$, implies that $\mathfrak{e}_{\overline{1}}$ is a Cartan subspace for the action of $N^{0}$ on $\mathfrak{f}_{\overline{1}}$.

Applying Theorem 3.3 just as in the proof of Theorem 3.4, one has that

$$
\left(\operatorname{ind}_{H}^{N^{0}} \mathbb{C}\right) \otimes S\left(\mathfrak{f}_{1}^{*}\right)^{N^{0}} \cong S\left(\mathfrak{f}_{1}^{*}\right)
$$

as graded right $S\left(\mathfrak{f}_{\overline{1}}^{*}\right)^{N^{0}}$-modules. Since the action of $N^{0}$ on $\mathfrak{f}_{\overline{1}}$ is polar, $S\left(\mathfrak{f}_{\overline{1}}^{*}\right)^{N^{0}}$ is a polynomial ring with an induced $N / N^{0}$ action. Since $N / N^{0}$ is a finite group and

$$
\left(S\left(\mathfrak{f}_{1}^{*}\right)^{N^{0}}\right)^{N / N^{0}} \cong S\left(\mathfrak{f}_{1}^{*}\right)^{N}
$$

is a polynomial ring, it follows that $N / N^{0}$ is a pseudoreflection group. By the classical theory of such groups GW, Theorem 12.4.7], multiplication gives an isomorphism,

$$
\left(\operatorname{ind}_{1}^{N / N^{0}} \mathbb{C}\right) \otimes\left(S\left(\mathfrak{f}_{1}^{*}\right)^{N^{0}}\right)^{N / N^{0}} \cong S\left(\mathfrak{f}_{1}^{*}\right)^{N^{0}}
$$

as graded right $S\left(\mathfrak{f}_{1}^{*}\right)^{N}$-modules. Therefore, combining these results, one has

$$
\begin{aligned}
S\left(\mathfrak{f}_{\overline{1}}^{*}\right) & \cong\left(\operatorname{ind}_{H}^{N^{0}} \mathbb{C}\right) \otimes S\left(\mathfrak{f}_{\overline{1}}^{*}\right)^{N^{0}} \\
& \cong\left(\operatorname{ind}_{H}^{N^{0}} \mathbb{C}\right) \otimes\left(\operatorname{ind}_{1}^{N / N^{0}} \mathbb{C}\right) \otimes S\left(\mathfrak{f}_{1}^{*}\right)^{N}, \\
& \cong\left(\operatorname{ind}_{H}^{N^{0}} \mathbb{C}\right) \otimes\left(\operatorname{ind}_{N^{0}}^{N} \mathbb{C}\right) \otimes S\left(\mathfrak{f}_{1}^{*}\right)^{N} .
\end{aligned}
$$

The action of $N$ on $\operatorname{ind}_{1}^{N / N^{0}} \mathbb{C}$ is by inflation through the canonical map $N \rightarrow$ $N / N^{0}$. Under this action $\operatorname{ind}_{1}^{N / N^{0}} \mathbb{C} \cong \operatorname{ind}_{N^{0}}^{N} \mathbb{C}$ as $N$-modules, yielding the last isomorphism. The action of $N \cong N^{0} \rtimes N / N^{0}$ on $\operatorname{ind}_{H}^{N^{0}} \mathbb{C}$ is as described in [Jan, I.3.8]. 
By the tensor identity and transitivity of induction [Jan, I.3.5-6], one obtains

$$
\begin{aligned}
S\left(\mathfrak{f}_{\overline{1}}^{*}\right) & \cong \operatorname{ind}_{N^{0}}^{N}\left(\operatorname{ind}_{H}^{N^{0}} \mathbb{C} \otimes \mathbb{C}\right) \otimes S\left(\mathfrak{f}_{1}^{*}\right)^{N} \\
& \cong\left(\operatorname{ind}_{N^{0}}^{N} \operatorname{ind}_{H}^{N^{0}} \mathbb{C}\right) \otimes S\left(\mathfrak{f}_{1}^{*}\right)^{N} \\
& \cong\left(\operatorname{ind}_{H}^{N} \mathbb{C}\right) \otimes S\left(\mathfrak{f}_{1}^{*}\right)^{N}
\end{aligned}
$$

This proves the desired result.

Finally, consider the case of the group $N^{\prime}=\operatorname{Norm}_{G_{\overline{0}}}\left(\mathfrak{e}_{\overline{1}}\right)$ acting on the Cartan subspace $\mathfrak{e}_{\overline{1}}$. Note that since $x_{0} \in \mathfrak{e}_{\overline{1}} \subseteq \mathfrak{f}_{\overline{1}}$, one has that $\operatorname{Stab}_{G_{\overline{0}}}\left(\mathfrak{e}_{\overline{1}}\right)=H$.

Theorem 3.6. Let $N^{\prime}=\operatorname{Norm}_{G_{\overline{0}}}\left(\mathfrak{e}_{\overline{1}}\right)$. Then there is a graded $N^{\prime}$-module $\mathcal{H} \subseteq S\left(\mathfrak{e}_{\overline{1}}^{*}\right)$ such that the multiplication map

$$
\mathcal{H} \otimes S\left(\mathfrak{e}_{1}^{*}\right)^{N^{\prime}} \rightarrow S\left(\mathfrak{e}_{1}^{*}\right)
$$

gives an isomorphism of graded right $S\left(\mathfrak{e}_{1}^{*}\right)^{N^{\prime}}$-modules. Furthermore, $\mathcal{H} \cong \operatorname{ind}_{H}^{N^{\prime}} \mathbb{C}$ as $N^{\prime}$-modules.

Proof. Since $W$ is a pseudoreflection group, one has that the multiplication map,

$$
\mathcal{H} \otimes S\left(\mathfrak{e}_{1}^{*}\right)^{W} \rightarrow S\left(\mathfrak{e}_{1}^{*}\right)
$$

gives an isomorphism as graded right $S\left(\mathfrak{e}_{1}^{*}\right)^{W}$-modules and where $\mathcal{H}=\operatorname{ind}_{1}^{W} \mathbb{C}[\mathrm{GW}$, Theorem 12.4.7]. However, viewing $\mathcal{H}$ as an $N^{\prime}$-module by inflation through the canonical quotient map $N^{\prime} \rightarrow N^{\prime} / H=W$, we have $\mathcal{H}=\operatorname{ind}_{H}^{N^{\prime}} \mathbb{C}$ as $N^{\prime}$-modules.

3.6. Taken together, Theorems 3.4 3.5 and 3.6 imply that when $\mathfrak{g}$ is both stable and polar the injective graded maps given in Theorems 3.1 (c) and 3.2(c) are of free $R$-modules of the same rank (namely, rank $\operatorname{dim} M^{H}$ ), where

$$
R:=S\left(\mathfrak{g}_{1}^{*}\right)^{G_{\overline{0}}} \cong S\left(\mathfrak{f}_{1}^{*}\right)^{N} \cong S\left(\mathfrak{e}_{1}^{*}\right)^{W} .
$$

In general such maps are proper injections but become isomorphisms over the field of fractions of $R$. However, one can apply a result of Panyushev $[\mathrm{Pan}$, Theorem 1] on covariants to show that in fact one need only invert a single element of $R$. Arguing just as in the proof of [ $\mathrm{Pan}$, Proposition 4], one has the following result.

Theorem 3.7. Let $\mathfrak{g}$ be stable and polar and let $M$ be a $G_{\overline{0}}$-module. Let $D^{\prime} \in R$ be such that for $y \in \mathfrak{g}_{\overline{1}}, D^{\prime}(y) \neq 0$ implies $y$ is generic. Then the graded $R$-module maps induced by restriction

$$
\rho: \operatorname{Hom}_{G_{\overline{0}}}\left(S\left(\mathfrak{g}_{\overline{1}}\right), M\right) \rightarrow \operatorname{Hom}_{N}\left(S\left(\mathfrak{f}_{\overline{1}}\right), M\right) \rightarrow \operatorname{Hom}_{N^{\prime}}\left(S\left(\mathfrak{e}_{\overline{1}}\right), M\right)
$$

are isomorphisms after one localizes at $D^{\prime}$.

Recall from Section 3.3 that, for the classical Lie superalgebras which are stable and polar considered in Sections 8.1 8.7, the set of generic points of $\mathfrak{g}_{\overline{1}}$ is precisely the principal open set defined by the discriminant of $W$. Therefore, in these cases the above theorem applies when one localizes at the discriminant. 


\section{Construction of Detecting subalgebras}

4.1. For the remainder of the paper we assume $\mathfrak{g}$ is stable and polar. Some results require this assumption, while others still hold under weaker hypotheses. To avoid technicalities and for clarity of exposition, we assume both.

We are now prepared to apply the invariant theory results of the previous section to our study of cohomology. In order to accomplish this goal we first construct two subsuperalgebras of $\mathfrak{g}$ which will detect the relative cohomology ring of $\mathfrak{g}$. This is done as follows. Since $\mathfrak{g}$ is stable one can fix a generic point $x_{0} \in \mathfrak{g}_{\overline{1}}$, and we let $H=G_{\overline{0}, x_{0}}$ and $N=N_{G_{\overline{0}}}(H)$.

Let $\mathfrak{f}_{\overline{1}}=\mathfrak{g}_{\overline{1}}^{H}$ as in the previous section. Let

$$
\mathfrak{f}_{\overline{0}}=\operatorname{Lie}(N) \subseteq \mathfrak{g}_{\overline{0}}
$$

and set

$$
\mathfrak{f}=\mathfrak{f}_{\overline{0}} \oplus \mathfrak{f}_{\overline{1}} \subseteq \mathfrak{g} .
$$

Since $\mathfrak{g}$ is polar, one can let $\mathfrak{e}_{\overline{1}} \subseteq \mathfrak{g}_{\overline{1}}$ be the Cartan subspace chosen in the previous section so that it satisfies (3.3.2). Let

$$
\mathfrak{e}_{\overline{0}}=\operatorname{Lie}(H) \subseteq \mathfrak{g}_{\overline{0}} .
$$

Set

$$
\mathfrak{e}=\mathfrak{e}_{\overline{0}} \oplus \mathfrak{e}_{\overline{1}} \subseteq \mathfrak{g}
$$

Recall (cf. [TY, 24.3.3-6]) that

$$
\operatorname{Lie}(H)=\left\{y \in \mathfrak{g}_{\overline{0}} \mid\left[y, x_{0}\right]=0\right\} .
$$

Theorem 4.1. Let $\mathfrak{g}$ be a classical Lie superalgebra which is stable and polar. Then there exist classical Lie subsuperalgebras $\mathfrak{f}$ and $\mathfrak{e}$ as constructed above, such that the restriction maps induce isomorphisms of graded superalgebras

$$
\mathrm{H}^{\bullet}\left(\mathfrak{g}, \mathfrak{g}_{\overline{0}} ; \mathbb{C}\right) \cong \mathrm{H}^{\bullet}\left(\mathfrak{f}, \mathfrak{f}_{\overline{0}} ; \mathbb{C}\right)^{N / N^{0}} \cong \mathrm{H}^{\bullet}\left(\mathfrak{e}, \mathfrak{e}_{\overline{0}} ; \mathbb{C}\right)^{W},
$$

where $N / N^{0}$ and $W$ are finite pseudoreflection groups. In particular, $\mathrm{H}^{\bullet}\left(\mathfrak{g}, \mathfrak{g}_{\overline{0}} ; \mathbb{C}\right)$ is isomorphic to a polynomial ring in $r:=\operatorname{dim} \mathfrak{e}_{\overline{1}}$ variables. Additionally, each cohomology ring is integral over the image(s) of res contained within it, and so all three rings have the same Krull dimension.

Proof. First we prove that if $y, z \in \mathfrak{f}_{\overline{1}}$, then $[y, z] \in \operatorname{Lie}(H)$. Note that by definition we have $x_{0} \in \mathfrak{f}_{\overline{1}}$ and $\left[x_{0}, x_{0}\right] \in \operatorname{Lie}(H)$. For $y \in \mathfrak{f}_{\overline{1}}$ the super version of the Jacobi identity implies

$$
0=\left[y,\left[x_{0}, x_{0}\right]\right]=\left[\left[y, x_{0}\right], x_{0}\right]-\left[x_{0},\left[y, x_{0}\right]\right]=2\left[\left[y, x_{0}\right], x_{0}\right] .
$$

Thus, $\left[y, x_{0}\right] \in \operatorname{Lie}(H)$. For the general case let $y, z \in \mathfrak{f}_{\overline{1}}$; then we have

$$
\left[[y, z], x_{0}\right]=\left[y,\left[z, x_{0}\right]\right]-\left[\left[y, x_{0}\right], z\right]=0,
$$

hence $[y, z] \in \operatorname{Lie}(H)$.

From the statement above it can be verified that $\mathfrak{f}$ and $\mathfrak{e}$ are Lie subsuperalgebras of $\mathfrak{g}$. Since $N$ and $H$ are reductive subgroups of $G_{\overline{0}}$, it follows that $\mathfrak{f}$ and $\mathfrak{e}$ are classical Lie superalgebras. Consequently we can apply Theorem 2.6 to the pairs $\left(\mathfrak{f}, \mathfrak{f}_{\overline{0}}\right)$ and $\left(\mathfrak{e}, \mathfrak{e}_{\overline{0}}\right)$ and reinterpret Theorems 3.1 and 3.2 to obtain the stated results. 
4.2. Injectivity of cochains. We can also consider the relationship between relative cohomology for $\mathfrak{g}, \mathfrak{f}$, and $\mathfrak{e}$ with coefficients in a finite dimensional supermodule other than the trivial supermodule.

Theorem 4.2. Let $M$ be a finite dimensional $\mathfrak{g}$-supermodule. Then restriction induces injective graded maps

$$
C^{d}\left(\mathfrak{g}, \mathfrak{g}_{\overline{0}} ; M\right) \hookrightarrow C^{d}\left(\mathfrak{f}, \mathfrak{f}_{\overline{0}} ; M\right)^{N / N^{0}} \hookrightarrow C^{d}\left(\mathfrak{e}, \mathfrak{e}_{\overline{0}} ; M\right)^{W} .
$$

Furthermore, all three cochain complexes are free $\mathrm{H}^{\bullet}\left(\mathfrak{g}, \mathfrak{g}_{0} ; \mathbb{C}\right)$-modules of rank $\operatorname{dim} M^{H}$.

Proof. First, recall that if $K$ is an algebraic group and $M_{1}$ and $M_{2}$ are $K$-modules, then $\operatorname{Hom}_{\operatorname{Lie}(K)}\left(M_{1}, M_{2}\right)=\operatorname{Hom}_{K^{0}}\left(M_{1}, M_{2}\right)$. Using this to reinterpret Theorems 3.1(c) and 3.2(c) one has the first result. The second statement follows from Theorems 3.4, 3.5, and 3.6.

4.3. In general the maps in Theorem 4.2 fail to be surjective. However, more can be said in certain cases.

Theorem 4.3. Let $\mathfrak{g}$ be a classical Lie superalgebra which is stable and polar. Furthermore, assume $\mathrm{H}^{\bullet}\left(\mathfrak{g}, \mathfrak{g}_{\overline{0}} ; \mathbb{C}\right)$ is isomorphic to a polynomial ring in one variable. Let $M$ be a finite dimensional $\mathfrak{g}$-supermodule. Then restriction induces an isomorphism

$$
\mathrm{H}^{d}\left(\mathfrak{g}, \mathfrak{g}_{\overline{0}} ; M\right) \cong \mathrm{H}^{d}\left(\mathfrak{f}, \mathfrak{f}_{\overline{0}} ; M\right)^{N / N^{0}} \cong \mathrm{H}^{d}\left(\mathfrak{e}, \mathfrak{e}_{\overline{0}} ; M\right)^{W}
$$

for $d \gg 0$.

Proof. First observe that if $R=\mathbb{C}[T]$ is a graded polynomial ring in one variable with $\operatorname{deg}(T)=t$ and $M \hookrightarrow N$ is an injective graded $R$-module homomorphism between two free $\mathbb{Z}$-graded $R$-modules of the same finite rank, then for $d \gg 0$ the map defines an isomorphism $M_{d} \cong N_{d}$, where $X_{d}$ denotes the $d$ th graded piece of the module $X$. To see this one can compare dimensions. Suppose that $U$ is a free graded $R$-module of rank $k$ :

$$
U=R u_{1} \oplus \cdots \oplus R u_{k} .
$$

Observe that for $d>\max \left\{\operatorname{deg}\left(u_{1}\right), \ldots, \operatorname{deg}\left(u_{k}\right)\right\}$ one has

$$
\operatorname{dim}\left(U_{d} \oplus \cdots \oplus U_{d+t-1}\right)=k .
$$

This, along with the fact that $\operatorname{dim}\left(M_{d}\right) \leq \operatorname{dim}\left(N_{d}\right)$, implies the claim.

Let us consider the first isomorphism in the statement of the theorem and leave the other nearly identical case to the reader. Combining Theorem 4.2 and the above observation one has

$$
C^{d}\left(\mathfrak{g}, \mathfrak{g}_{\overline{0}} ; M\right) \cong C^{d}\left(\mathfrak{f}, \mathfrak{f}_{\overline{0}} ; M\right)^{N / N^{0}}
$$

for $d \gg 0$. The result then follows from the fact that the differentials in (2.3.1) are $N / N^{0}$ homomorphisms, and, since $N / N^{0}$ is a finite group, taking $N / N^{0}$ invariants is an exact functor.

As seen from Table 1, the above stability result holds for $\mathfrak{g l}(1 \mid n), \mathfrak{q}(1), D(2,1 ; \alpha)$, $G(3)$, and $F(4)$, among others. 
4.4. Localization of cohomology. Let $D^{\prime}$ be the element in $\mathrm{H}^{\bullet}\left(\mathfrak{g}, \mathfrak{g}_{0} ; \mathbb{C}\right)$ chosen in Theorem 3.7 If $X$ is an $\mathrm{H}^{\bullet}\left(\mathfrak{g}, \mathfrak{g}_{\overline{0}} ; \mathbb{C}\right)$-module, write $X_{D^{\prime}}$ to denote the localization of $X$ at $D^{\prime}$. The following theorem relates the various relative cohomology groups under localization.

Theorem 4.4. Let $M$ be a finite dimensional $\mathfrak{g}$-supermodule. Then restriction induces isomorphisms

$$
\mathrm{H}^{\bullet}\left(\mathfrak{g}, \mathfrak{g}_{\overline{0}} ; M\right)_{D^{\prime}} \cong\left(\mathrm{H}^{\bullet}\left(\mathfrak{f}, \mathfrak{f}_{\overline{0}} ; M\right)^{N / N^{0}}\right)_{D^{\prime}} \cong\left(\mathrm{H}^{\bullet}\left(\mathfrak{e}, \mathfrak{e}_{\overline{0}} ; M\right)^{W}\right)_{D^{\prime}} .
$$

Proof. Applying Theorem 3.7 to (4.2.1), we see that

$$
C^{d}\left(\mathfrak{g}, \mathfrak{g}_{\overline{0}} ; M\right)_{D^{\prime}} \cong\left(C^{d}\left(\mathfrak{f}, \mathfrak{f}_{\overline{0}} ; M\right)^{N / N^{0}}\right)_{D^{\prime}} \cong\left(C^{d}\left(\mathfrak{e}, \mathfrak{e}_{\overline{0}} ; M\right)^{W}\right)_{D^{\prime}} .
$$

The differentials are graded $\mathrm{H}^{\bullet}\left(\mathfrak{g}, \mathfrak{g}_{\overline{0}} ; \mathbb{C}\right)$-module homomorphisms, and localization is an exact functor; thus one obtains the stated result.

\section{REPRESENTATION THEORY FOR DETECTING SUbALGEBRAS}

In the previous section we constructed a Lie subsuperalgebra $\mathfrak{e}$ of $\mathfrak{g}$ which detects cohomology. This subalgebra will play an important role in defining rank varieties for finite dimensional supermodules. The definition of the rank varieties depends on understanding the representation theory of $\mathfrak{e}$. We develop the theory at this point.

5.1. The category $\mathcal{F}_{\left(\mathfrak{e}, \mathfrak{e}_{\overline{0}}\right)}$. In this subsection we investigate the general representation theory of the Lie superalgebra $\mathfrak{e}$ defined in Section 4.1. The key fact which will be used below without comment is that

$$
\left[\mathfrak{e}_{\overline{0}}, \mathfrak{e}_{\overline{1}}\right]=0 ;
$$

that is, elements of $\mathfrak{e}_{\overline{0}}$ and $\mathfrak{e}_{\overline{1}}$ commute.

Lemma 5.1. Let $M$ be a finite dimensional e-supermodule. Then

$$
\left(U(\mathfrak{e}) \otimes_{U\left(\mathfrak{e}_{\overline{0}}\right)} M\right)^{*} \cong U(\mathfrak{e}) \otimes_{U\left(\mathfrak{e}_{\overline{0}}\right)} M^{*}
$$

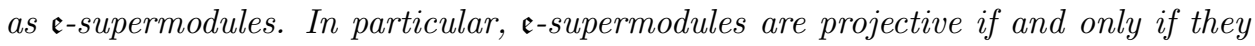
are injective.

Proof. To prove (5.1.1) we first consider the special case when $M=\mathbb{C}$, the trivial supermodule. Fix a basis $x_{1}, \ldots, x_{r}$ for $\mathfrak{e}_{\overline{1}}$. By the PBW theorem for Lie superalgebras, if $N:=U(\mathfrak{e}) \otimes_{U\left(\mathfrak{e}_{\overline{0}}\right)} \mathbb{C}$, then the elements

$$
x_{1}^{d_{1}} \cdots x_{r}^{d_{r}} \otimes 1,
$$

where $d_{1}, \ldots, d_{r} \in\{0,1\}$, form a basis for $N$. Define a dual basis for $N^{*}$ by the formula

$$
\left[x_{1}^{d_{1}} \cdots x_{r}^{d_{r}} \otimes 1\right]^{*}\left(x_{1}^{e_{1}} \cdots x_{r}^{e_{r}} \otimes 1\right)=\delta_{d_{1}, e_{1}} \cdots \delta_{d_{r}, e_{r}} .
$$

Define the $\mathfrak{e}$-supermodule homomorphism

$$
\alpha: U(\mathfrak{e}) \otimes_{U\left(\mathfrak{e}_{\overline{0}}\right)} \mathbb{C} \rightarrow\left(U(\mathfrak{e}) \otimes_{U\left(\mathfrak{e}_{\overline{0}}\right)} \mathbb{C}\right)^{*}
$$

to be the map induced by Frobenius reciprocity from the $\mathfrak{e}_{\overline{0}}$-supermodule homomorphism $\mathbb{C} \rightarrow\left(U(\mathfrak{e}) \otimes_{U\left(\mathfrak{e}_{\overline{0}}\right)} \mathbb{C}\right)^{*}$ given by $1 \mapsto\left[x_{1} \cdots x_{r} \otimes 1\right]^{*}$. 
We will prove $\alpha$ is an isomorphism. Before doing so, let us set some notation. Given a tuple $d=\left(d_{1}, \ldots, d_{r}\right) \in\{0,1\}^{r}$, let $|d|=d_{1}+\cdots+d_{r}$ and let $\tilde{d}_{i}=1-d_{i}$. We claim

$$
\alpha\left(x_{1}^{d_{1}} \cdots x_{r}^{d_{r}} \otimes 1\right)= \pm\left[x_{1}^{\tilde{d}_{1}} \cdots x_{r}^{\tilde{d}_{r}} \otimes 1\right]^{*} .
$$

Note that if $\alpha$ satisfies (5.1.2), then it is an isomorphism.

To prove (5.1.2), one inducts on $|d|$, with the case $|d|=0$ being clear from the definition of $\alpha$. Now consider the case $|d|>0$. Fix $k$ so that $d_{k}=1$ and $d_{i}=0$ for $i=1, \ldots, k-1$. Then,

$$
\begin{aligned}
\alpha\left(x_{1}^{d_{1}} \cdots x_{r}^{d_{r}} \otimes 1\right) & =(-1)^{\bar{\alpha}} x_{k} \alpha\left(x_{1}^{d_{1}} \cdots x_{k-1}^{d_{k-1}} x_{k}^{\tilde{d}_{k}} x_{k+1}^{d_{k+1}} \cdots x_{r}^{d_{r}} \otimes 1\right) \\
& = \pm x_{k}\left[x_{1}^{\tilde{d}_{1}} \cdots x_{k-1}^{\tilde{d}_{k-1}} x_{k}^{d_{k}} x_{k+1}^{\tilde{d}_{k+1}} \cdots x_{r}^{\tilde{d}_{r}} \otimes 1\right]^{*}
\end{aligned}
$$

where the second equality is by the inductive assumption. Now on the one hand we have

$$
\begin{aligned}
& \left(x_{k}\left[x_{1}^{\tilde{d}_{1}} \cdots x_{k}^{d_{k}} \cdots x_{r}^{\tilde{d}_{r}} \otimes 1\right]^{*}\right)\left(x_{1}^{e_{1}} \cdots x_{r}^{e_{r}} \otimes 1\right) \\
& = \pm\left[x_{1}^{\tilde{d}_{1}} \cdots x_{k}^{d_{k}} \cdots x_{r}^{\tilde{d}_{r}} \otimes 1\right]^{*}\left(x_{k} x_{1}^{e_{1}} \cdots x_{r}^{e_{r}} \otimes 1\right) \\
& = \pm\left[x_{1}^{\tilde{d}_{1}} \cdots x_{k}^{d_{k}} \cdots x_{r}^{\tilde{d}_{r}} \otimes 1\right]^{*}\left(x_{1}^{e_{1}} \cdots x_{k-1}^{e_{k-1}} x_{k}^{e_{k}+1} x_{k+1}^{e_{k+1}} \cdots x_{r}^{e_{r}} \otimes 1\right) \\
& = \begin{cases} \pm \delta_{\tilde{d}_{1}, e_{1}} \cdots \delta_{d_{k}, e_{k}+1} \cdots \delta_{\tilde{d}_{r}, e_{r}} & \text { if } e_{k}=0, \\
0 & \text { if } e_{k}=1 .\end{cases}
\end{aligned}
$$

Note that the second case follows from the observation that

$$
x_{k}^{2}=\frac{1}{2}\left[x_{k}, x_{k}\right] \in \mathfrak{e}_{\overline{0}} .
$$

On the other hand,

$$
\left[x_{1}^{\tilde{d}_{1}} \cdots x_{r}^{\tilde{d}_{r}} \otimes 1\right]^{*}\left(x_{1}^{e_{1}} \cdots x_{r}^{e_{r}} \otimes 1\right)=\delta_{\tilde{d}_{1}, e_{1}} \cdots \delta_{\tilde{d}_{k}, e_{k}} \cdots \delta_{\tilde{d}_{r}, e_{r}} .
$$

Using the assumption that $d_{k}=1$, one can verify that these two expressions are equal up to sign. This proves that $\alpha$ defines an isomorphism. The general case then follows by an argument using the tensor identity.

Now consider the second statement. Let $P$ be a finite dimensional projective $\mathfrak{e}$-supermodule. Then by Frobenius reciprocity we have an even surjective map

$$
N:=U(\mathfrak{e}) \otimes_{U\left(\mathfrak{e}_{\overline{0}}\right)} P \rightarrow P .
$$

Since $P$ is projective, we have an even isomorphism $N \cong P \oplus U$. Dualizing and applying (5.1.1) we see that $P^{*}$ is a direct summand of the supermodule $U(\mathfrak{e}) \otimes_{U\left(\mathfrak{e}_{\overline{0}}\right)}$ $P^{*}$. However, $U(\mathfrak{e}) \otimes_{U\left(\mathfrak{e}_{\overline{0}}\right)} P^{*}$ is projective since $P^{*}$ is an object of $\mathcal{F}_{\left(\mathfrak{e}, \mathfrak{e}_{\overline{0}}\right)}$, hence a projective $\mathfrak{e}_{\overline{0}}$-supermodule, and induction is exact. Therefore $P^{*}$ is both projective and injective, hence so is $P$.

Lemma 5.2. Let $M$ be a finite dimensional $\mathfrak{e}$-supermodule, and let

$$
M=M_{1} \oplus \cdots \oplus M_{t}
$$

be a decomposition into $\mathfrak{e}_{\overline{0}}$-isotypic components. Then (5.1.3) is a decomposition into e-supermodules. 
Proof. Let $L$ be a simple $\mathfrak{e}^{-}$-supermodule which appears as a direct summand of the isotypic component $M_{k}$ of $M$. Let $y \in \mathfrak{e}_{\overline{1}}$. Then there is an $\mathfrak{e}_{\overline{0}}$-supermodule homomorphism $L \rightarrow M$ given by left multiplication by $y$. By the irreducibility of $L$ the image of $L$ under this map is either zero or isomorphic to $L$. In either case, $y L \subseteq M_{k}$. Therefore, for any $y \in \mathfrak{e}_{\overline{1}}$ we have $y M_{k} \subseteq M_{k}$. This, along with the observation that the decomposition (5.1.3) respects the $\mathbb{Z}_{2}$-grading of $M$, implies the decomposition (5.1.3) is as $\mathfrak{e}$-supermodules.

Suppose an $\mathfrak{e}$-supermodule $M$ contains one and only one simple $\mathfrak{e}_{\overline{0}}$-supermodule as a composition factor, say $L$. In this case we say $M$ is of type $L$. Let us write $\mathcal{F}_{L}$ for the full subcategory of $\mathcal{F}_{\left(\mathfrak{e}, \mathfrak{e}_{\overline{0}}\right)}$ consisting of all $\mathfrak{e}$-supermodules of type $L$. Applying Lemma 5.2 we have the following direct sum decomposition of the category $\mathcal{F}_{\left(\mathfrak{e}, \mathfrak{e}_{\overline{0}}\right)}$ :

$$
\mathcal{F}_{\left(\mathfrak{e}, \mathfrak{e}_{\overline{0}}\right)}=\bigoplus_{L} \mathcal{F}_{L}
$$

where the sum is over a complete, irredundant set of simple $\mathfrak{e}_{\overline{0}}^{-}$-supermodules. We refer to the subcategory $\mathcal{F}_{L}$ as the block of $\mathcal{F}_{\left(\mathfrak{e}, \mathfrak{e}_{\overline{0}}\right)}$ of type $L$.

Lemma 5.3. Let $L$ be a finite dimensional simple $\mathfrak{e}_{\overline{0}}$-supermodule. The finite dimensional simple $\mathfrak{e}$-supermodules which lie in $\mathcal{F}_{L}$ are precisely the composition factors of

$$
U(\mathfrak{e}) \otimes_{U\left(\mathfrak{e}_{\overline{0}}\right)} L .
$$

In particular, the block $\mathcal{F}_{L}$ contains only finitely many simples.

Proof. Let $T$ be a simple $\mathfrak{e}$-supermodule lying in $\mathcal{F}_{L}$. Then by Frobenius reciprocity we have a surjective map

$$
\bigoplus U(\mathfrak{e}) \otimes_{U\left(\mathfrak{e}_{\overline{0}}\right)} L \rightarrow T
$$

where the sum runs over some finite index set. Thus $T$ is a composition factor of the supermodule $U(\mathfrak{e}) \otimes_{U\left(\mathfrak{e}_{\overline{0}}\right)} L$.

5.2. Representations for rank one Lie superalgebras. The definition of rank varieties in Section 6.3 depends on restricting to rank one Lie superalgebras. Here we compile some basic results on finite dimensional representations of these superalgebras. Let $\langle x\rangle$ be a Lie superalgebra generated by a single odd vector, $x$. There are the following two possibilities for $\langle x\rangle$ :

I. We have $[x, x]=0$. Then $\operatorname{dim}\langle x\rangle_{\overline{0}}=0, \operatorname{dim}\langle x\rangle_{\overline{1}}=1$, and $\langle x\rangle$ is a one dimensional abelian Lie superalgebra concentrated in degree $\overline{1}$.

II. We have $h:=[x, x] \neq 0$. Then $\operatorname{dim}\langle x\rangle_{\overline{0}}=1, \operatorname{dim}\langle x\rangle_{\overline{1}}=1$, and $\langle x\rangle$ is isomorphic to the Lie superalgebra $\mathfrak{q}(1)$.

Let us first consider Case I. The universal enveloping superalgebra $U(\langle x\rangle)$ is spanned by 1 and $x$ and is subject to the relation $x^{2}=0$. It is easy to see that the trivial module is the only finite dimensional simple $U(\langle x\rangle)$-supermodule and that $U(\langle x\rangle)$ is the projective cover of $\mathbb{C}$. Furthermore, a direct calculation verifies that $U(\langle x\rangle)^{*} \cong U(\langle x\rangle)$ as $U(\langle x\rangle)$-supermodules, so $U(\langle x\rangle)$ is also injective. From this we see that $U(\langle x\rangle)$ is the unique self extension of $\mathbb{C}$ and that $\{U(\langle x\rangle), \mathbb{C}\}$ is a complete, irredundant list of indecomposable $U(\langle x\rangle)$-supermodules.

Now consider Case II. By the PBW theorem for Lie superalgebras $U(\langle x\rangle)$ is spanned by monomials of the form

$$
x^{r} h^{s}
$$


where $r \in\{0,1\}$ and $s \in \mathbb{Z}_{\geq 0}$. In particular, one has an induction functor from the category of finite dimensional $\langle x\rangle_{\overline{0}}$-supermodules to the category of finite dimensional $\langle x\rangle$-supermodules given by

$$
\operatorname{ind}_{\langle x\rangle_{\overline{0}}}^{\langle x\rangle_{\overline{0}}} M=U(\langle x\rangle) \otimes_{U\left(\langle x\rangle_{\overline{0}}\right)} M .
$$

Furthermore, this functor is both left adjoint to restriction and exact because $U(\langle x\rangle)$ is a free right $U\left(\langle x\rangle_{\overline{0}}\right)$-supermodule.

From the PBW theorem one sees that $U\left(\langle x\rangle_{\overline{0}}\right)$ is isomorphic to the polynomial ring in one variable. Consequently, the category of finite dimensional $U\left(\langle x\rangle_{\overline{0}}\right)$ supermodules is semisimple, and we obtain the following classification of the simple $\langle x\rangle_{0}$-supermodules: for each $\lambda \in \mathbb{C}$ let $\mathbb{C}_{\lambda}$ denote $\mathbb{C}$ viewed as an $\langle x\rangle_{\overline{0}}$-supermodule concentrated in degree $\overline{0}$ where $h$ acts by the scalar $\lambda$; then the set $\left\{\mathbb{C}_{\lambda} \mid \lambda \in \mathbb{C}\right\}$ is a complete irredundant set of simple $\langle x\rangle_{\overline{0}}$-supermodules. Furthermore, since $U\left(\langle x\rangle_{\overline{0}}\right)$ is semisimple, these are projective as well. Since induction is exact, the $\langle x\rangle$-supermodule

$$
P(\lambda):=\operatorname{ind}_{\langle x\rangle_{\overline{0}}}^{\left\langle x \mathbb{C}_{\lambda}\right.}=U(\langle x\rangle) \otimes_{U\left(\langle x\rangle_{\overline{0}}\right)} \mathbb{C}_{\lambda}
$$

is projective for each $\lambda \in \mathbb{C}$.

One the one hand, by Frobenius reciprocity every simple $U(\langle x\rangle)$-supermodule is the quotient of $P(\lambda)$ for some $\lambda$. On the other hand, a direct calculation verifies that $P(\lambda)$ is a simple supermodule if and only if $\lambda \neq 0$. To warn the reader that there are subtleties, note that $P(\lambda)$ is the direct sum of two one dimensional $U(\langle x\rangle)$ modules when $\lambda \neq 0$. In the case when $\lambda=0$ we have that $P(\lambda)$ has simple head and socle, each isomorphic to the trivial supermodule.

Now consider indecomposables. By the above discussion, all the simple supermodules lie in separate blocks. Furthermore, since all simple supermodules except the trivial supermodule are projective, the only indecomposable in a non-principal block is the simple supermodule itself.

Now consider the principal block. Applying Theorem 2.6 we see that

$$
\operatorname{dim}\left(\operatorname{Ext}_{\mathcal{F}_{(\langle x\rangle,\langle x\rangle \overline{0})}}^{1}(\mathbb{C}, \mathbb{C})\right)=1 .
$$

That is, $P(0)$ is the unique (up to isomorphism) self extension of the trivial supermodule. Consequently, $P(0)$ is self dual, hence injective. From this observation it follows that $P(0)$ and the trivial supermodule are the only indecomposable supermodules in the principal block.

We summarize the above analysis in the following proposition. This proposition can also be obtained using an alternative approach which involves Clifford superalgebras (cf. [BKN, Section 5]).

Proposition 5.4. If $\langle x\rangle$ is as in Case I above, then the following statements about the category of finite dimensional $\langle x\rangle$-supermodules hold:

(a) The trivial supermodule $L(0):=\mathbb{C}$ is the only simple supermodule.

(b) The projective cover of $L(0)$ is $P(0):=U(\langle x\rangle)$.

(c) The supermodule $P(0)$ is self dual, hence injective.

(d) The set $\{P(0), L(0)\}$ is a complete set of indecomposable supermodules.

If $\langle x\rangle$ is as in Case II above, then the following statements about the category of finite dimensional $\langle x\rangle$-supermodules hold. Given $\lambda \in \mathbb{C}$, let $P(\lambda)$ be as in (5.2.1). Let $L(\lambda)$ denote the head of $P(\lambda)$. 
(e) The set $\{L(\lambda) \mid \lambda \in \mathbb{C}\}$ is a complete set of simple $\langle x\rangle$-supermodules.

(f) For all $\lambda \in \mathbb{C}, P(\lambda)$ is the projective cover of $L(\lambda)$.

(g) If $\lambda \neq 0$, then $L(\lambda)=P(\lambda)$.

(h) For all $\lambda \in \mathbb{C}, P(\lambda)$ is dual to $P(-\lambda)$, hence injective.

(i) The set $\{L(\lambda) \mid \lambda \in \mathbb{C}\} \cup\{P(0)\}$ is a complete set of indecomposable supermodules.

We remark that in both cases the supermodules $P(\lambda)$ always satisfy

$$
\operatorname{dim}\left(P(\lambda)_{\overline{0}}\right)=\operatorname{dim}\left(P(\lambda)_{\overline{1}}\right)=1 .
$$

In particular, $P(\lambda)$ is always two dimensional.

\section{Support VARIETIES}

6.1. In this section we define the notion of the support variety of a finite dimensional $\mathfrak{g}$-supermodule $M$ and study the properties of these varieties. Let $\mathfrak{a}$ be a classical Lie superalgebra (e.g., $\mathfrak{a}$ could be one of $\mathfrak{g}, \mathfrak{f}$, or $\mathfrak{e}$ from the previous sections). Let $M$ and $N$ be $\mathfrak{a}$-supermodules in the category $\mathcal{F}_{\left(\mathfrak{a}, \mathfrak{a}_{\overline{0}}\right)}$. Recall that by Theorem 2.7 one knows that

$$
\operatorname{Ext}_{\mathcal{F}_{\left(\mathfrak{a}, \mathfrak{a}_{\overline{0}}\right)}}(M, N) \cong \mathrm{H}^{\bullet}\left(\mathfrak{a}, \mathfrak{a}_{\overline{0}} ; M^{*} \otimes N\right)
$$

is a finitely generated $\mathrm{H}^{\bullet}\left(\mathfrak{a}, \mathfrak{a}_{\overline{0}} ; \mathbb{C}\right)$-module. Let

$$
I_{\left(\mathfrak{a}, \mathfrak{a}_{\overline{0}}\right)}(M, N)=\operatorname{Ann}_{\mathrm{H}} \bullet{\left(\mathfrak{a}, \mathfrak{a}_{\overline{0}} ; \mathbb{C}\right)}\left(\mathrm{H}^{\bullet}\left(\mathfrak{a}, \mathfrak{a}_{\overline{0}} ; M^{*} \otimes N\right)\right),
$$

the annihilator ideal of this module. We define the relative support variety of the pair $(M, N)$ to be

$$
\mathcal{V}_{\left(\mathfrak{a}, \mathfrak{a}_{\overline{0}}\right)}(M, N)=\operatorname{MaxSpec}\left(\mathrm{H}^{\bullet}\left(\mathfrak{a}, \mathfrak{a}_{\overline{0}} ; \mathbb{C}\right) / I_{\left(\mathfrak{a}, \mathfrak{a}_{\overline{0}}\right)}(M, N)\right),
$$

the maximal ideal spectrum of the quotient of $\mathrm{H}^{\bullet}\left(\mathfrak{a}, \mathfrak{a}_{\overline{0}} ; \mathbb{C}\right)$ by $I_{\left(\mathfrak{a}, \mathfrak{a}_{\overline{0}}\right)}(M, N)$. For short, when $M=N$, write

$$
\begin{aligned}
I_{\left(\mathfrak{a}, \mathfrak{a}_{\overline{0}}\right)} & =I_{\left(\mathfrak{a}, \mathfrak{a}_{\overline{0}}\right)}(M, M), \\
\mathcal{V}_{\left(\mathfrak{a}, \mathfrak{a}_{\overline{0}}\right)}(M) & =\mathcal{V}_{\left(\mathfrak{a}, \mathfrak{a}_{\overline{0}}\right)}(M, M) .
\end{aligned}
$$

We call $\mathcal{V}_{\left(\mathfrak{a}, \mathfrak{a}_{\overline{0}}\right)}(M)$ the support variety of $M$. Let us remark that, just as for finite groups, $I_{\left(\mathfrak{a}, \mathfrak{a}_{\overline{0}}\right)}(M)$ is precisely the annihilator ideal of the identity element of $\mathrm{H}^{\bullet}\left(\mathfrak{a}, \mathfrak{a}_{\overline{0}} ; M^{*} \otimes M\right)$ viewed as a ring under the Yoneda product.

Set $r:=\operatorname{dim} \mathfrak{e}_{\overline{1}}$. If $\mathfrak{a}$ equals $\mathfrak{g}, \mathfrak{f}$, or $\mathfrak{e}$, then $\mathcal{V}_{\left(\mathfrak{a}, \mathfrak{a}_{\overline{0}}\right)}(M) \subseteq \mathcal{V}_{\left(\mathfrak{a}, \mathfrak{a}_{\overline{0}}\right)}(\mathbb{C}) \cong \mathbb{A}^{r}$. That is, $\mathcal{V}_{\left(\mathfrak{a}, \mathfrak{a}_{\overline{0}}\right)}(M)$ can naturally be viewed as a conical (since the defining ideal is graded) affine subvariety of $\mathbb{A}^{r}$.

Recall that when $\mathfrak{g}$ is stable and polar one has canonical restriction maps

$$
\mathrm{H}^{\bullet}\left(\mathfrak{g}, \mathfrak{g}_{\overline{0}} ; \mathbb{C}\right) \rightarrow \mathrm{H}^{\bullet}\left(\mathfrak{f}, \mathfrak{f}_{\overline{0}} ; \mathbb{C}\right) \rightarrow \mathrm{H}^{\bullet}\left(\mathfrak{e}, \mathfrak{e}_{\overline{0}} ; \mathbb{C}\right)
$$

which induce maps, which we call res*:

$$
\mathcal{V}_{\left(\mathfrak{e}, \mathfrak{e}_{\overline{0}}\right)}(\mathbb{C}) \rightarrow \mathcal{V}_{\left(\mathfrak{f}, \mathfrak{f}_{\overline{0}}\right)}(\mathbb{C}) \rightarrow \mathcal{V}_{\left(\mathfrak{g}, \mathfrak{g}_{\overline{0}}\right)}(\mathbb{C})
$$

By Theorem 4.1 one then has

$$
\mathbb{A}^{r} \cong \mathcal{V}_{\left(\mathfrak{e}, \mathfrak{e}_{\overline{0}}\right)}(\mathbb{C}) / W \cong \mathcal{V}_{\left(\mathfrak{f}, \bar{f}_{\overline{0}}\right)}(\mathbb{C}) /\left(N / N^{0}\right) \cong \mathcal{V}_{\left(\mathfrak{g}, \mathfrak{g}_{\overline{0}}\right)}(\mathbb{C})
$$

Furthermore, if $M$ is a finite dimensional $\mathfrak{g}$-supermodule, then res* restricts to give maps

$$
\mathcal{V}_{\left(\mathfrak{e}, \mathfrak{e}_{\overline{0}}\right)}(M) \rightarrow \mathcal{V}_{\left(\mathfrak{f}, \mathfrak{f}_{\overline{0}}\right)}(M) \rightarrow \mathcal{V}_{\left(\mathfrak{g}, \mathfrak{g}_{\overline{0}}\right)}(M)
$$


Since the first two varieties are stable under the action of $W$ and $N / N^{0}$, respectively, by applying (6.1.2) we have the following embeddings of varieties induced by res*:

$$
\begin{aligned}
\mathcal{V}_{\left(\mathfrak{e}, \mathfrak{e}_{\overline{0}}\right)}(M) / W & \cong \operatorname{res}^{*}\left(\mathcal{V}_{\left(\mathfrak{e}, \mathfrak{e}_{\overline{0}}\right)}(M)\right) \subseteq \mathcal{V}_{\left(\mathfrak{g}, \mathfrak{g}_{\overline{0}}\right)}(M), \\
\mathcal{V}_{\left(\mathfrak{f}, \mathfrak{f}_{\overline{0}}\right)}(M) /\left(N / N^{0}\right) & \cong \operatorname{res}^{*}\left(\mathcal{V}_{\left(\mathfrak{f}, \mathfrak{f}_{\overline{0}}\right)}(M)\right) \subseteq \mathcal{V}_{\left(\mathfrak{g}, \mathfrak{g}_{\overline{0}}\right)}(M) .
\end{aligned}
$$

6.2. Relating support varieties. Naturally one would like to better understand the relationship between the varieties $\mathcal{V}_{\left(\mathfrak{g}, \mathfrak{g}_{\bar{g}}\right)}(M), \mathcal{V}_{\left(\mathfrak{f}, \mathfrak{f}_{\overline{0}}\right)}(M)$, and $\mathcal{V}_{\left(\mathfrak{e}, \mathfrak{e}_{\overline{0}}\right)}(M)$ for a finite dimensional $\mathfrak{g}$-supermodule $M$. In particular, one would like to understand the maps given in (6.1.3). It may be that these maps are surjective, at least for suitably nice supermodules. In any case, one can use results from Section 4 to gain some insight.

Theorem 6.1. Assume $\mathfrak{g}$ is a classical Lie superalgebra which is stable and polar. Assume further that $\mathrm{H}^{\bullet}\left(\mathfrak{g}, \mathfrak{g}_{0} ; \mathbb{C}\right)$ is isomorphic to a polynomial ring in one variable. Let $M$ be a finite dimensional $\mathfrak{g}$-supermodule. Then res* gives the following equalities:

$$
\operatorname{res}^{*}\left(\mathcal{V}_{\left(\mathfrak{e}, \mathfrak{e}_{\overline{0}}\right)}(M)\right)=\operatorname{res}^{*}\left(\mathcal{V}_{\left(\mathfrak{f}, \mathfrak{f}_{\overline{0}}\right)}(M)\right)=\mathcal{V}_{\left(\mathfrak{g}, \mathfrak{g}_{\overline{0}}\right)}(M)
$$

Proof. We consider the map res* $: \mathcal{V}_{\left(\mathfrak{e}, \mathfrak{e}_{\overline{0}}\right)}(M) \rightarrow \mathcal{V}_{\left(\mathfrak{g}, \mathfrak{g}_{\overline{0}}\right)}(M)$. The other case follows similarily.

In light of (6.1.3), it suffices to prove res* is surjective. Also note that by the "lying over" theorem for maximal ideals [AM, Theorem 5.10, Corollary 5.8], $\operatorname{res}^{*}\left(\mathcal{V}_{\left(\mathfrak{e}, \mathfrak{e}_{\overline{0}}\right)}(M)\right)$ is precisely the subvariety of $\mathcal{V}_{\left(\mathfrak{g}, \mathfrak{g}_{\overline{0}}\right)}(\mathbb{C})$ defined by the ideal $\operatorname{res}^{-1}\left(I_{\left(\mathfrak{e}, \mathfrak{e}_{\overline{0}}\right)}(M)\right)$. We claim that

$$
I_{\left(\mathfrak{g}, \mathfrak{g}_{\overline{0}}\right)}(M) \subseteq \operatorname{res}^{-1}\left(I_{\left(\mathfrak{e}, \mathfrak{e}_{\overline{0}}\right)}(M)\right) \subseteq \sqrt{I_{\left(\mathfrak{g}, \mathfrak{g}_{\overline{0}}\right)}(M)},
$$

where the latter is the radical ideal of $I_{\left(\mathfrak{g}, \mathfrak{g}_{\overline{0}}\right)}(M)$. This suffices to prove the desired assertion as the radical of an ideal defines the same variety as the ideal itself.

First, by Theorem 4.3, one can fix $N>0$ so that the restriction map

$$
\mathrm{H}^{d}\left(\mathfrak{g}, \mathfrak{g}_{\overline{0}} ; M\right) \rightarrow \mathrm{H}^{d}\left(\mathfrak{e}, \mathfrak{e}_{\overline{0}} ; M\right)^{W} \subseteq \mathrm{H}^{d}\left(\mathfrak{e}, \mathfrak{e}_{\overline{0}} ; M\right)
$$

is an isomorphism for all $d \geq N$.

The first inclusion of (6.2.1) is clear from the description of $I_{\left(\mathfrak{g}, \mathfrak{g}_{\overline{0}}\right)}(M)$ as the annihilator of the identity of $\mathrm{H}^{\bullet}\left(\mathfrak{g}, \mathfrak{g}_{0} ; M^{*} \otimes M\right)$. On the other hand, let $x \in$ $\operatorname{res}^{-1}\left(I_{\left(\mathfrak{e}, \mathfrak{e}_{\overline{0}}\right)}(M)\right)$ and let $m \in \mathrm{H}^{p}\left(\mathfrak{g} ; \mathfrak{g}_{\overline{0}} ; M^{*} \otimes M\right)$ for some $p \geq 0$. Then $\operatorname{res}(x) \operatorname{res}(m)$ $=0$ so

$$
\operatorname{res}\left(x^{N} m\right)=\operatorname{res}(x)^{N} \operatorname{res}(m)=0 .
$$

However, by our choice of $N$ the map res is injective, so $x^{N} m=0$. But since $m$ was arbitrary, we see that $x^{N} \in I_{\left(\mathfrak{g}, \mathfrak{g}_{\overline{0}}\right)}(M)$; that is, $x \in \sqrt{I_{\left(\mathfrak{g}, \mathfrak{g}_{\overline{0}}\right)}(M)}$.

Before continuing, we recall from commutative algebra (cf. AM, Chapter 3, Exercise 1]) that if $R$ is a commutative ring, $S \subseteq R$ a multiplicatively closed set, and $U$ a finitely generated $R$-module, then $S^{-1} U=0$ if and only if $s U=0$ for some $s \in S$. In particular, this implies that if $\mathfrak{m} \in \mathcal{V}_{(\mathfrak{g}, \mathfrak{g} \overline{0})}(\mathbb{C})$, then $\mathfrak{m} \in \mathcal{V}_{\left(\mathfrak{g}, \mathfrak{g}_{\overline{0}}\right)}(M)$ if and only if $\mathrm{H}^{\bullet}\left(\mathfrak{g}, \mathfrak{g}_{\overline{0}} ; M^{*} \otimes M\right)_{\mathfrak{m}} \neq 0$.

In general, (6.1.3) is "generically" an isomorphism in the following sense. Let $D^{\prime} \in \mathrm{H}^{\bullet}\left(\mathfrak{g}, \mathfrak{g}_{0} ; \mathbb{C}\right)$ be as chosen in Theorem 3.7 and let

$$
\mathcal{O}_{D^{\prime}}=\left\{a \in \mathcal{V}_{\left(\mathfrak{g}, \mathfrak{g}_{\overline{0}}\right)}(\mathbb{C}) \mid D^{\prime}(a) \neq 0\right\}=\left\{\mathfrak{m} \in \operatorname{MaxSpec}\left(\mathrm{H}^{\bullet}\left(\mathfrak{g}, \mathfrak{g}_{\overline{0}} ; \mathbb{C}\right)\right) \mid D^{\prime} \notin \mathfrak{m}\right\},
$$


the principal dense open set defined by $D^{\prime}$. In particular, as remarked in Section 3.3 . for the classical Lie superalgebras considered in Sections 8.1 8.7 which are stable and polar, one can take $D^{\prime}$ to be the discriminant of $W$. Then $\mathcal{O}_{D^{\prime}}$ is precisely the image in $\mathcal{V}_{\left(\mathfrak{g}, \mathfrak{g}_{\overline{0}}\right)}(\mathbb{C})=\mathfrak{g}_{\overline{1}} / G_{\overline{0}}$ of the set of generic points of $\mathfrak{g}_{\overline{1}}$.

Theorem 6.2. Let $M$ be a finite dimensional $\mathfrak{g}$-supermodule. Then

$$
\operatorname{res}^{*}\left(\mathcal{V}_{\left(\mathfrak{e}, \mathfrak{e}_{\overline{0}}\right)}(M)\right) \cap \mathcal{O}_{D^{\prime}}=\operatorname{res}^{*}\left(\mathcal{V}_{\left(\mathfrak{f}, \mathfrak{f}_{\overline{0}}\right)}(M)\right) \cap \mathcal{O}_{D^{\prime}}=\mathcal{V}_{\left(\mathfrak{g}, \mathfrak{g}_{\overline{0}}\right)}(M) \cap \mathcal{O}_{D^{\prime}}
$$

Proof. We prove res* $\left(\mathcal{V}_{\left(\mathfrak{e}, \mathfrak{e}_{\overline{0}}\right)}(M)\right) \cap \mathcal{O}_{D^{\prime}}=\mathcal{V}_{\left(\mathfrak{g}, \mathfrak{g}_{\overline{0}}\right)}(M) \cap \mathcal{O}_{D^{\prime}}$ and leave the other case for the reader.

It is clear that res* $\left(\mathcal{V}_{\left(\mathfrak{e}, \mathfrak{e}_{\overline{0}}\right)}(M)\right) \cap \mathcal{O}_{D^{\prime}} \subseteq \mathcal{V}_{\left(\mathfrak{g}, \mathfrak{g}_{\overline{0}}\right)}(M) \cap \mathcal{O}_{D^{\prime}}$. On the other hand, let $\mathfrak{m} \in \mathcal{V}_{\left(\mathfrak{g}, \mathfrak{g}_{\overline{0}}\right)}(M) \cap \mathcal{O}_{D^{\prime}}$. That is, $\mathrm{H}^{\bullet}\left(\mathfrak{g}, \mathfrak{g}_{\overline{0}} ; M^{*} \otimes M\right)_{\mathfrak{m}} \neq 0$ and $D^{\prime} \notin \mathfrak{m}$. Theorem 4.4 implies that

$$
0 \neq \mathrm{H}^{\bullet}\left(\mathfrak{g}, \mathfrak{g}_{\overline{0}} ; M^{*} \otimes M\right)_{\mathfrak{m}} \cong\left(\mathrm{H}^{\bullet}\left(\mathfrak{e}, \mathfrak{e}_{\overline{0}} ; M^{*} \otimes M\right)^{W}\right)_{\mathfrak{m}} \subseteq \mathrm{H}^{\bullet}\left(\mathfrak{e}, \mathfrak{e}_{\overline{0}} ; M^{*} \otimes M\right)_{\mathfrak{m}} .
$$

This implies the reverse inclusion.

6.3. Rank varieties. Given Theorems 6.1 and 6.2, we are motivated to more closely study the support varieties associated to the detecting subsuperalgebra $\mathfrak{e}$. The goal of this subsection is to define rank varieties and prove an analogue the Avrunin-Scott Theorem (first conjectured for finite groups by Carlson). Our approach is based on ideas used in the context of restricted Lie algebras by Friedlander and Parshall [FP1, Theorem 2.7].

Let $M$ be a finite dimensional $\mathfrak{e}$-supermodule. As a matter of notation, given a homogeneous element $x \in \mathfrak{e}$, let $\langle x\rangle$ denote the Lie subsuperalgebra generated by $x$. Define the rank variety of $M$ to be

$$
\mathcal{V}_{\mathfrak{e}}^{\text {rank }}(M)=\left\{x \in \mathfrak{e}_{\overline{1}} \mid M \text { is not projective as an }\langle x\rangle \text {-supermodule }\right\} \cup\{0\} .
$$

Observe that

$$
\mathcal{V}_{\mathfrak{e}}^{\text {rank }}(\mathbb{C})=\mathfrak{e}_{\overline{1}}
$$

We record some basic properties of rank varietes in the following proposition.

Proposition 6.3. Let $M$ and $N$ be finite dimensional $\mathfrak{e}$-supermodules. Then:

(a) $\mathcal{V}_{\mathfrak{e}}^{\text {rank }}(M \otimes N)=\mathcal{V}_{\mathfrak{e}}^{\text {rank }}(M) \cap \mathcal{V}_{\mathfrak{e}}^{\text {rank }}(N)$.

(b) $\mathcal{V}_{\mathfrak{e}}^{\text {rank }}\left(M^{*}\right)=\mathcal{V}_{\mathfrak{e}}^{\text {rank }}(M)$

(c) $\mathcal{V}_{\mathfrak{e}}^{\text {rank }}\left(M^{*} \otimes M\right)=\mathcal{V}_{\mathfrak{e}}^{\text {rank }}(M)$.

Proof. (a) From Proposition 5.4 one observes that $x \in \mathcal{V}_{\mathfrak{e}}^{\text {rank }}(M)$ if and only if $M$ contains a trivial direct summand as an $\langle x\rangle$-supermodule. A direct calculation verifies that if $P(\lambda)$ and $P(\mu)$ are two indecomposable projective $\langle x\rangle$-supermodules, then

$$
P(\lambda) \otimes P(\mu) \cong P(\lambda+\mu) \oplus P(\lambda+\mu)
$$

as $\langle x\rangle$-supermodules. The result follows from these observations.

(b)-(c) From Proposition 5.4 we see that for any $x \in \mathfrak{e}_{\overline{1}}, M^{*}$ is projective as an $\langle x\rangle$-supermodule if and only if $M$ is projective. This implies the first result. The second result then follows from (a).

Theorem 6.4. Let $M$ be a finite dimensional $\mathfrak{e}$-supermodule. Then there is an isomorphism

$$
\mathcal{V}_{\mathfrak{e}}^{\mathrm{rank}}(\mathbb{C}) \rightarrow \mathcal{V}_{\left(\mathfrak{e}, \mathfrak{e}_{\overline{0}}\right)}(\mathbb{C})
$$


which restricts to give an isomorphism

$$
\mathcal{V}_{\mathfrak{e}}^{\text {rank }}(M) \rightarrow \mathcal{V}_{\left(\mathfrak{e}, \mathfrak{e}_{\overline{0}}\right)}(M) .
$$

Proof. The isomorphism $\mathcal{V}_{\left(\mathfrak{e}, \mathfrak{e}_{\overline{0}}\right)}(\mathbb{C}) \cong \mathcal{V}_{\mathfrak{e}}^{\text {rank }}(\mathbb{C})$ is a direct consequence of Hilbert's Nullstellensatz as $\mathrm{H}^{\bullet}\left(\mathfrak{e}, \mathfrak{e}_{\overline{0}} ; \mathbb{C}\right) \cong S\left(\mathfrak{e}_{1}^{*}\right)$.

Now let $M$ be a finite dimensional $\mathfrak{e}$-supermodule. To prove the second statement, we identify $\mathcal{V}_{\left(\mathfrak{e}, \mathfrak{e}_{\overline{0}}\right)}(\mathbb{C})$ with $\mathfrak{e}_{\overline{1}}$ using the above isomorphism and show that $x \in \mathcal{V}_{\left(\mathfrak{e}, \mathfrak{e}_{\overline{0}}\right)}(M)$ if and only if $x \in \mathcal{V}_{\mathfrak{e}}^{\operatorname{rank}}(M)$.

First, let $0 \neq x \in \mathcal{V}_{\mathfrak{e}}^{\text {rank }}(M)$. Using Proposition 5.4 one can verify directly that the isomorphism $\mathcal{V}_{\langle x\rangle}^{\operatorname{rank}}(\mathbb{C}) \rightarrow \mathcal{V}_{\left(\langle x\rangle,\langle x\rangle_{\overline{0}}\right)}(\mathbb{C})$ restricts to give an isomorphism

$$
\mathcal{V}_{\langle x\rangle}^{\mathrm{rank}}(M) \stackrel{\cong}{\rightrightarrows} \mathcal{V}_{\left(\langle x\rangle,\langle x\rangle_{\overline{0}}\right)}(M) .
$$

By choosing coordinates one can verify that the restriction map

$$
\operatorname{res}^{*}: \mathcal{V}_{\left(\langle x\rangle,\langle x\rangle_{\overline{0}}\right)}(\mathbb{C})=\mathbb{C} x \rightarrow \mathcal{V}_{\left(\mathfrak{e}, \mathfrak{e}_{\overline{0}}\right)}(\mathbb{C})=\mathfrak{e}_{\overline{1}}
$$

is the injective map which has as its image the line which goes through 0 and $x$. By restricting this map one then has the injective map

$$
\operatorname{res}^{*}: \mathcal{V}_{\left(\langle x\rangle,\langle x\rangle_{\overline{0}}\right)}(M) \hookrightarrow \mathcal{V}_{\left(\mathfrak{e}, \mathfrak{e}_{\overline{0}}\right)}(M)
$$

with image equal to the line through 0 and $x$. Therefore, if $0 \neq x \in \mathcal{V}_{\mathfrak{e}}^{\text {rank }}(M)$, then $x \in \mathcal{V}_{\left(\mathfrak{e}, \mathfrak{e}_{\overline{0}}\right)}(M)$.

Conversely, assume $x \notin \mathcal{V}_{\mathfrak{e}}^{\text {rank }}(M)$. Then $M^{*} \otimes M$ is projective as an $\langle x\rangle$ supermodule, and so $\mathrm{H}^{q}\left(\langle x\rangle,\langle x\rangle_{\overline{0}} ; M^{*} \otimes M\right)=0$ for $q>0$. Let $I=\langle x\rangle+\mathfrak{e}_{\overline{0}}$, a graded ideal of $\mathfrak{e}$. Viewing $I$ as a Lie subsuperalgebra of $\mathfrak{e}$ and $M^{*} \otimes M$ as an $I$-supermodule by restriction, we wish to prove $\mathrm{H}^{q}\left(I, I_{\overline{0}} ; M^{*} \otimes M\right)=0$ for $q>0$.

One can observe that the cochains used to define $\mathrm{H}^{\bullet}\left(I, I_{\overline{0}} ; M^{*} \otimes M\right)$ in Section 2.3 can be obtained by taking the $\mathfrak{e}_{\overline{0}}$ invariants of the cochains which define $\mathrm{H}^{\bullet}\left(\langle x\rangle,\langle x\rangle_{\overline{0}} ; M^{*} \otimes M\right)$. Since the cochains which define $\mathrm{H}^{\bullet}\left(\langle x\rangle,\langle x\rangle_{\overline{0}} ; M^{*} \otimes M\right)$ are finitely semisimple as $\mathfrak{e}_{\overline{0}}$-supermodules, taking $\mathfrak{e}_{\overline{0}}$ invariants is an exact functor. Furthermore, using that $[z, x]=0$ for any $z \in \mathfrak{e}_{\overline{0}}$, one sees that the differentials are $\mathfrak{e}_{\overline{0}}$-supermodule homomorphisms. Taken together, this discussion implies $\mathrm{H}^{q}\left(I, I_{\overline{0}} ; M^{*} \otimes M\right) \cong \mathrm{H}^{q}\left(\langle x\rangle,\langle x\rangle_{\overline{0}} ; M^{*} \otimes M\right)^{\mathfrak{e}_{\overline{0}}}=0$ for all $q>0$.

There is a Hochschild-Serre spectral sequence with $E_{2}$ page

$$
E_{2}^{p, q}=\mathrm{H}^{p}\left(\mathfrak{e} / I, 0 ; \mathrm{H}^{q}\left(I, I_{\overline{0}} ; M^{*} \otimes M\right)\right) \Rightarrow \mathrm{H}^{p+q}\left(\mathfrak{e}, \mathfrak{e}_{\overline{0}} ; M^{*} \otimes M\right)
$$

(cf. [Fuk, Sections 1.5-1.6]). Since $\mathrm{H}^{q}\left(I, I_{\overline{0}} ; M^{*} \otimes M\right)=0$ for $q>0$, one has

$$
\mathrm{H}^{p}\left(\mathfrak{e} / I, 0 ;\left(M^{*} \otimes M\right)^{I}\right) \cong \mathrm{H}^{p}\left(\mathfrak{e}, \mathfrak{e}_{\overline{0}} ; M^{*} \otimes M\right)
$$

for all $p \geq 0$. Then by Theorem 2.7. $\mathrm{H}^{\bullet}\left(\mathfrak{e}, \mathfrak{e}_{\overline{0}} ; M^{*} \otimes M\right)$ is finitely generated as a module for

$$
\mathrm{H}^{\bullet}(\mathfrak{e} / I, 0 ; \mathbb{C}) \cong S\left(\left((\mathfrak{e} / I)_{\overline{1}}\right)^{*}\right) \cong S\left(\left(\mathfrak{e}_{\overline{1}} / \mathbb{C} x\right)^{*}\right)
$$

Let

$$
\begin{aligned}
N & =\mathrm{H}^{\bullet}\left(\mathfrak{e}, \mathfrak{e}_{\overline{0}} ; M^{*} \otimes M\right) \otimes_{S\left(\mathfrak{e}_{\overline{1}}^{*}\right)} S\left(\mathfrak{e}_{\overline{1}}^{*}\right) /\left(\left(\mathfrak{e}_{\overline{1}} / \mathbb{C} x\right)^{*} S\left(\mathfrak{e}_{\overline{1}}^{*}\right)\right) \\
& \cong \mathrm{H}^{\bullet}\left(\mathfrak{e}, \mathfrak{e}_{\overline{0}} ; M^{*} \otimes M\right) \otimes_{S\left(\left(\mathfrak{e}_{\overline{1}} / \mathbb{C} x\right)^{*}\right)} \mathbb{C} .
\end{aligned}
$$

From commutative algebra (e.g. AM, Chapter 3, Ex. 19(vi)]) we have that

$$
\operatorname{Supp}(N):=\left\{\mathfrak{m} \in \mathcal{V}_{\left(\mathfrak{e}, \mathfrak{e}_{\overline{0}}\right)}(\mathbb{C}) \mid N_{\mathfrak{m}} \neq 0\right\}=\mathcal{V}_{\left(\mathfrak{e}, \mathfrak{e}_{\overline{\mathfrak{e}}}\right)}(M) \cap \operatorname{res}^{*}\left(\mathcal{V}_{\left(\langle x\rangle,\langle x\rangle_{\overline{0}}\right)}(\mathbb{C})\right)
$$


However, since $\mathrm{H}^{\bullet}\left(\mathfrak{e}, \mathfrak{e}_{\overline{0}} ; M^{*} \otimes M\right)$ is a finitely generated $S\left(\left(\mathfrak{e}_{\overline{1}} / \mathbb{C} x\right)^{*}\right)$-module, $N$ is finite dimensional. This implies $\operatorname{Supp}(N)$ is a finite set. On the other hand, $\operatorname{Supp}(N)$ is conical. Therefore, $\operatorname{Supp}(N)=\{0\}$. That is, since $\operatorname{res}^{*}\left(\mathcal{V}_{\left(\langle x\rangle,\langle x\rangle_{\overline{0}}\right)}(\mathbb{C})\right)=$ $\mathbb{C} x, x \notin \mathcal{V}_{\left(\mathfrak{e}, \mathfrak{e}_{\overline{0}}\right)}(M)$.

6.4. One can use the rank variety description of $\mathcal{V}_{\left(\mathfrak{e}, \mathfrak{e}_{\overline{0}}\right)}(M)$ along with Lemmas 5.1 and 5.3 to prove the analogue of well known results in the theory of support varieties. In most cases the classical arguments from finite groups and restricted Lie algebras apply here as well. For these standard arguments we refer the reader to Ben, FP1].

Corollary 6.5. Let $M$ be a finite dimensional e-supermodule. If $\operatorname{dim} M_{\overline{0}} \neq \operatorname{dim} M_{\overline{1}}$ (e.g. when $M$ is odd dimensional), then

$$
\mathcal{V}_{\left(\mathfrak{e}, \mathfrak{e}_{\overline{0}}\right)}(M)=\mathcal{V}_{\left(\mathfrak{e}, \mathfrak{e}_{\overline{0}}\right)}(\mathbb{C}) .
$$

Proof. This follows from the rank variety description of $\mathcal{V}_{\left(\mathfrak{e}, \mathfrak{e}_{\overline{0}}\right)}(M)$. Namely, fix $0 \neq x \in \mathfrak{e}_{\overline{1}}$. By Proposition [5.4, the projective indecomposable $\langle x\rangle$-supermodules $P$ satisfy $\operatorname{dim} P_{\overline{0}}=\operatorname{dim} P_{\overline{1}}=1$. Thus if $M$ is a projective $\langle x\rangle$-supermodule, then $\operatorname{dim} M_{\overline{0}}=\operatorname{dim} M_{\overline{1}}$. As this contradicts what is assumed of $M, x \in \mathcal{V}_{\mathfrak{e}}^{\operatorname{rank}}(M)$. Therefore $\mathcal{V}_{\left(\mathfrak{e}, \mathfrak{e}_{\overline{0}}\right)}(M)=\mathcal{V}_{\mathfrak{e}}^{\operatorname{rank}}(M)=\mathfrak{e}_{\overline{1}}=\mathcal{V}_{\left(\mathfrak{e}, \mathfrak{e}_{\overline{0}}\right)}(\mathbb{C})$.

Before continuing, let us recall the following basic notions in homological algebra. If $\mathfrak{a}$ is a Lie superalgebra, then the complexity of a finite dimensional $\mathfrak{a}$-supermodule $M$ is the rate of growth of a minimal projective resolution of $M$ (cf. Ben, Sec. 5.3]). Given a finite dimensional a-supermodule $M$, let $P(M)$ denote the projective cover of $M$. Recalling that we assume $P(M)$ maps onto $M$ by an even supermodule homomorphism, one defines $\Omega(M)=\Omega^{1}(M)$ to be the kernel of the aforementioned homomorphism. For $n>1$ recursively define $\Omega^{n}(M)$ to be $\Omega\left(\Omega^{n-1}(M)\right)$.

The following theorem, taken together with Proposition 6.3. shows that support varieties for finite dimensional $\mathfrak{e}$-supermodules satisfy the desirable properties of a support variety theory.

Theorem 6.6. If $M, M_{1}, M_{2}, M_{3}$, and $N$ are finite dimensional $\mathfrak{e}$-supermodules, then:

(a) The complexity of $M$ equals $\operatorname{dim} \mathcal{V}_{\left(\mathfrak{e}, \mathfrak{e}_{\overline{0}}\right)}(M)$.

(b) $M$ is projective if and only if $\mathcal{V}_{\left(\mathfrak{e}, \mathfrak{e}_{\overline{0}}\right)}(M)=\{0\}$.

(c) $\mathcal{V}_{\left(\mathfrak{e}, \mathfrak{e}_{\overline{0}}\right)}\left(M_{1} \oplus M_{2}\right)=\mathcal{V}_{\left(\mathfrak{e}, \mathfrak{e}_{\overline{0}}\right)}\left(M_{1}\right) \cup \mathcal{V}_{\left(\mathfrak{e}, \mathfrak{e}_{\overline{0}}\right)}\left(M_{2}\right)$.

(d) If

$$
0 \rightarrow M_{1} \rightarrow M_{2} \rightarrow M_{3} \rightarrow 0
$$

is a short exact sequence of $\mathfrak{e}$-supermodules, then

$$
\mathcal{V}_{\left(\mathfrak{e}, \mathfrak{e}_{\overline{0}}\right)}\left(M_{i}\right) \subseteq \mathcal{V}_{\left(\mathfrak{e}, \mathfrak{e}_{\overline{0}}\right)}\left(M_{j}\right) \cup \mathcal{V}_{\left(\mathfrak{e}, \mathfrak{e}_{\overline{0}}\right)}\left(M_{k}\right)
$$

where $\{i, j, k\}=\{1,2,3\}$.

(e) For any $n \geq 1, \mathcal{V}_{\left(\mathfrak{e}, \mathfrak{e}_{\overline{0}}\right)}\left(\Omega^{n}(M)\right)=\mathcal{V}_{\left(\mathfrak{e}, \mathfrak{e}_{\overline{0}}\right)}(M)$.

(f) If $\mathcal{V}_{\left(\mathfrak{e}, \mathfrak{e}_{\overline{0}}\right)}\left(M_{1}\right) \cap \mathcal{V}_{\left(\mathfrak{e}, \mathfrak{e}_{\overline{0}}\right)}\left(M_{2}\right)=\{0\}$, then for any finite dimensional $\mathfrak{e}$-supermodule $N$ the short exact sequence

$$
0 \rightarrow M_{1} \rightarrow N \rightarrow M_{2} \rightarrow 0
$$

splits. 
Proof. Parts (a) and (b) are proved by the same arguments used for restricted Lie algebras [FP1, Propositions 1.5, 3.2]. Part (c) follows immediately from the rank variety description, and part (d) follows from the rank variety description along with Proposition 5.4. Part (e) follows from part (d) and the definition of $\Omega^{n}(M)$. Part (f) follows just as in the classical case using parts (a) and (d).

Just as for finite groups, one has $\mathrm{H}^{p}\left(\mathfrak{e}, \mathfrak{e}_{\overline{0}} ; \mathbb{C}\right) \cong \operatorname{Hom}_{\mathfrak{e}}\left(\Omega^{p}(\mathbb{C}), \mathbb{C}\right)$. Given $\zeta \in$ $\mathrm{H}^{p}\left(\mathfrak{e}, \mathfrak{e}_{\overline{0}} ; \mathbb{C}\right)$ we let

$$
L_{\zeta}=\operatorname{Ker}\left(\zeta: \Omega^{p}(\mathbb{C}) \rightarrow \mathbb{C}\right) \subseteq \Omega^{p}(\mathbb{C}) .
$$

As in the classical theory of support varieties (cf. [FP1, Lemma 4.2]), the importance of the supermodule $L_{\zeta}$ is that one can explicitly calculate its support variety. In particular, these supermodules allow one to prove the following realization theorem.

Theorem 6.7. Let $X$ be a closed conical subvariety of $\mathcal{V}_{\left(\mathfrak{e}, \mathfrak{e}_{\overline{0}}\right)}(\mathbb{C})$. There is a finite dimensional $\mathfrak{e}$-supermodule $M$ such that

$$
\mathcal{V}_{\left(\mathfrak{e}, \mathfrak{e}_{\overline{0}}\right)}(M)=X
$$

Proof. First, given $\zeta \in \mathrm{H}^{p}\left(\mathfrak{e}, \mathfrak{e}_{0} ; \mathbb{C}\right)$, one can compute the support variety of $L_{\zeta}$ as follows. From the graded version of Schanuel's Lemma, $\Omega^{p}(\mathbb{C}) \cong P \oplus \Omega_{\langle x\rangle}^{p}(\mathbb{C})$ as $\langle x\rangle$-supermodules, where $\Omega_{\langle x\rangle}^{p}(\mathbb{C})$ denotes $\Omega^{p}(\mathbb{C})$ for the trivial $\langle x\rangle$-supermodule and $P$ is some projective $\langle x\rangle$-supermodule. By Proposition $5.4, \Omega_{\langle x\rangle}^{p}(\mathbb{C}) \cong \mathbb{C}$. With these facts in hand one can argue just as for finite groups and restricted Lie algebras (cf. [FP1, Lemma 4.1]) to prove

$$
\mathcal{V}_{\left(\mathfrak{e}, \mathfrak{e}_{\overline{0}}\right)}\left(L_{\zeta}\right)=\mathcal{Z}(\zeta):=\left\{x \in \mathfrak{e}_{\overline{1}} \mid \zeta(x)=0\right\} .
$$

Now let $I=\left(\zeta_{1}, \ldots, \zeta_{t}\right) \subseteq \mathrm{H}^{\bullet}\left(\mathfrak{e}, \mathfrak{e}_{0} ; \mathbb{C}\right)$ be a homogeneous ideal which defines $X$. That is, $\zeta_{1}, \ldots, \zeta_{t}$ are homogeneous elements of $\mathrm{H}^{\bullet}\left(\mathfrak{e}, \mathfrak{e}_{\overline{0}} ; \mathbb{C}\right)$ and

$$
X=\mathcal{Z}\left(\zeta_{1}\right) \cap \cdots \cap \mathcal{Z}\left(\zeta_{t}\right) .
$$

Applying (6.4.2) and Proposition 6.3(a) one has

$$
\mathcal{V}_{\left(\mathfrak{e}, \mathfrak{e}_{\overline{0}}\right)}\left(L_{\zeta_{1}} \otimes \cdots \otimes L_{\zeta_{t}}\right)=\mathcal{Z}\left(\zeta_{1}\right) \cap \cdots \cap \mathcal{Z}\left(\zeta_{t}\right)=X
$$

We also have the following connectedness result.

Theorem 6.8. Suppose that $M$ is a finite dimensional $\mathfrak{e}$-supermodule such that

$$
\mathcal{V}_{\left(\mathfrak{e}, \mathfrak{e}_{\overline{0}}\right)}(M)=X_{1} \cup X_{2},
$$

where $X_{1}$ and $X_{2}$ are non-zero closed conical subvarieties with $X_{1} \cap X_{2}=\{0\}$. Then there are finite dimensional $\mathfrak{e}$-supermodules $M_{1}$ and $M_{2}$ such that $\mathcal{V}_{\left(\mathfrak{e}, \mathfrak{e}_{\overline{0}}\right)}\left(M_{1}\right)=X_{1}$, $\mathcal{V}_{\left(\mathfrak{e}, \mathfrak{e}_{\overline{0}}\right)}\left(M_{2}\right)=X_{2}$, and $M \cong M_{1} \oplus M_{2}$.

In particular, if $M$ is indecomposable, then the projectivization of the conical variety $\mathcal{V}_{\left(\mathfrak{e}, \mathfrak{e}_{\overline{0}}\right)}(M)$ is connected.

Proof. One can argue just as in the case of finite groups (cf. [Car]), using (6.4.2), Proposition 6.3. and Theorem 6.6. 


\section{Defect, ATyPiCAlity AND SUPERDimension}

7.1. Defect. Let $\mathfrak{g}$ be a classical Lie superalgebra and $\mathfrak{t}$ be a maximal torus contained in $\mathfrak{g}_{\overline{0}}$. Let $\Phi$ be the set of roots with respect to $\mathfrak{t}$. We have that $\Phi=\Phi_{\overline{0}} \cup \Phi_{\overline{1}}$, where $\Phi_{\overline{0}}$ (resp. $\Phi_{\overline{1}}$ ) is the set of even roots (resp. odd roots). The positive roots will be denoted by $\Phi^{+}$and the negative roots by $\Phi^{-}$. Set $\Phi_{\overline{0}}^{ \pm}=\Phi_{\overline{0}} \cap \Phi^{ \pm}$and $\Phi_{\overline{1}}^{ \pm}=\Phi_{\overline{1}} \cap \Phi^{ \pm}$. If $\mathfrak{g}$ is a basic classical Lie superalgebra (cf. Section 2.1), then Kac and Wakimoto [KW, §2] define the defect of $\mathfrak{g}$, denoted by $\operatorname{def}(\mathfrak{g})$, to be the dimension of a maximal isotropic subspace in the $\mathbb{R}$-span of $\Phi$.

The defects for the various simple basic classical Lie superalgebras are as follows [DS, Section 4]: $\operatorname{def}(\mathfrak{s l}(m \mid n))=\min (m, n), \operatorname{def}(\mathfrak{p s l}(n \mid n))=n, \operatorname{def}(\mathfrak{o s p}(2 m+1 \mid 2 n))$ $=\operatorname{def}(\mathfrak{o s p}(2 m \mid 2 n))=\min (m, n)$, and the exceptional Lie superalgebras $D(2,1 ; \alpha)$, $G(3)$, and $F(4)$ all have defect 1 . The following theorem demonstrates that one can realize this numerical defect using relative cohomology, support varieties, or the detecting subalgebra $\mathfrak{e}$.

Theorem 7.1. Let $\mathfrak{g}$ be a basic classical simple Lie superalgebra. Moreover, assume that $\mathfrak{g} ¥ \mathfrak{p s l}(n \mid n)$. The following numbers are equal:

(a) $\operatorname{def}(\mathfrak{g})$;

(b) $\operatorname{dim} \mathrm{H}^{\bullet}\left(\mathfrak{g}, \mathfrak{g}_{\overline{0}} ; \mathbb{C}\right)$;

(c) $\operatorname{dim} \mathcal{V}_{\left(\mathfrak{g}, \mathfrak{g}_{\overline{0}}\right)}(\mathbb{C})$.

Furthermore, if $\mathfrak{g}$ is stable and polar, then the above are also equal to the following numbers:

(d) $\operatorname{dim} H^{\bullet}\left(\mathfrak{a}, \mathfrak{a}_{\overline{0}} ; \mathbb{C}\right)$, where $\mathfrak{a}=\mathfrak{f}$ or $\mathfrak{e}$;

(e) $\operatorname{dim} \mathcal{V}_{\left(\mathfrak{a}, \mathfrak{a}_{\overline{0}}\right)}(\mathbb{C})$, where $\mathfrak{a}=\mathfrak{f}$ or $\mathfrak{e} ;$

(f) $\operatorname{dim} \mathcal{V}_{\mathfrak{e}}^{\operatorname{rank}}(\mathbb{C})$

(g) $\operatorname{dim} \mathfrak{e}_{\overline{1}}$.

Proof. The equality of (a)-(c) follows by verifying that the defects listed above equal the dimensions presented in Table 1 of the Appendix. That these coincide with the dimensions listed in (d)-(g) follow from the results in Sections 3 and 4 .

The above theorem indicates that one could naturally extend the definition of defect cohomologically to arbitrary classical Lie superalgebras by $\operatorname{setting} \operatorname{def}(\mathfrak{g})$ to be the Krull dimension of $\mathrm{H}^{\bullet}\left(\mathfrak{g}, \mathfrak{g}_{0} ; \mathbb{C}\right)$. In particular, the new definition would allow one to define the notion of defect for the simple classical Lie superalgebras of types $P(n)$ and $Q(n)$. Also, let us remark that the definition given by Kac and Wakimoto would differ from this new definition for the Lie superalgebra $\mathfrak{p s l}(n \mid n)$. Namely, $\operatorname{def}(\mathfrak{p s l}(n \mid n))=\operatorname{def}(\mathfrak{g l}(n \mid n))=n$, whereas

$$
\operatorname{dim} \mathrm{H}^{\bullet}\left(\mathfrak{p s l}(n \mid n), \mathfrak{p s l}(n \mid n)_{\overline{0}} ; \mathbb{C}\right)=n+1 \neq \operatorname{dim} \mathrm{H}^{\bullet}\left(\mathfrak{g l l}(n \mid n), \mathfrak{g l}(n \mid n)_{\overline{0}} ; \mathbb{C}\right)=n
$$

7.2. Atypicality. Let $\mathfrak{g}$ be a basic classical Lie superalgebra as above and let $(-,-)$ denote the bilinear form. Let $\lambda \in \mathfrak{t}^{*}$ be a weight. The atypicality of $\lambda$ is the maximal number of linearly independent, mutually orthogonal, positive isotropic roots $\alpha \in \Phi^{+}$such that $(\lambda+\rho, \alpha)=0$, where $\rho=\frac{1}{2}\left(\sum_{\alpha \in \Phi_{\overline{0}}^{+}} \alpha-\sum_{\alpha \in \Phi_{\overline{1}}^{+}} \alpha\right)$. Note that $\operatorname{atyp}(\lambda) \leq \operatorname{def}(\mathfrak{g})$. Let $L(\lambda)$ be a simple finite dimensional $\mathfrak{g}$-supermodule with highest weight $\lambda$. The atypicality of $L(\lambda)$, denoted by atyp $(L(\lambda))$, is defined to be $\operatorname{atyp}(\lambda)$. We present the following strong conjecture. 
Conjecture 7.2. Let $\mathfrak{g}$ be a simple basic classical Lie superalgebra which is stable and polar and let $L(\lambda)$ be a finite dimensional simple $\mathfrak{g}$-supermodule. Then

$$
\operatorname{atyp}(L(\lambda))=\operatorname{dim} \mathcal{V}_{\mathfrak{e}}^{\text {rank }}(L(\lambda)) .
$$

There is the following evidence in favor of the conjecture. Using results in $\mathrm{KW}$ one can verify the conjecture for all $\mathfrak{g}$ which have defect one. The conjecture also holds in the case when the atypicality of the simple $\mathfrak{g}$-supermodule $L(\lambda)$ is zero. Namely, atyp $(L(\lambda))=0$ implies $L(\lambda)$ is a projective $U(\mathfrak{g})$-supermodule (cf. Kac2, Theorem 1]), thus projective as a $U(\mathfrak{e})$-supermodule. This in turn implies $\mathcal{V}_{\mathfrak{e}}^{\operatorname{rank}}(L(\lambda))=\{0\}$. Finally, in the case of $\mathfrak{g}=\mathfrak{g l}(m \mid n)$, one can use translation functor techniques from DS, Ser5] to prove $\operatorname{atyp}(L(\lambda))=\operatorname{dim} \mathcal{V}_{\mathfrak{e}}^{\text {rank }}(L(\lambda))$ for any finite dimensional simple $\mathfrak{g}$-supermodule $L(\lambda)$ (cf. [BKN]).

7.3. Kac-Wakimoto Conjecture. Let $M$ be a supermodule. The superdimension of $M$ is defined to be $\operatorname{sdim} M=\operatorname{dim} M_{\overline{0}}-\operatorname{dim} M_{\overline{1}}$. Kac and Wakimoto give the following conjecture relating the superdimension of simple finite dimensional $\mathfrak{g}$ supermodules with the atypicality of the module and the defect of $\mathfrak{g}$.

Conjecture 7.3 (ㅉ, Conjecture 3.1]). Let $\mathfrak{g}$ be a simple basic classical Lie superalgebra and $L(\lambda)$ be a finite dimensional simple $\mathfrak{g}$-module. Then $\operatorname{sdim} L(\lambda)=0$ if and only if $\operatorname{atyp}(L(\lambda))<\operatorname{def}(\mathfrak{g})$.

We will now indicate how our conjecture given in Section 7.2 and the KacWakimoto Conjecture are interrelated. On the one hand, the validity of our conjecture implies one direction of the Kac-Wakimoto Conjecture. Suppose that $\operatorname{sdim} L(\lambda)$ $\neq 0$. Then by Corollary 6.5, $\operatorname{dim} \mathcal{V}_{\mathfrak{e}}^{\text {rank }}(L(\lambda))=\operatorname{dim} \mathfrak{e}_{\overline{1}}$. So by using Conjecture 7.2 and Theorem 7.1 atyp $(L(\lambda))=\operatorname{def}(\mathfrak{g})$. Note that this implication of the KacWakimoto conjecture was recently verified for the simple classical contragradient Lie superalgebras [DS, Lemma 7.1]. On the other hand, assume that the KacWakimoto Conjecture is valid. Then our conjecture is true for all simple $\mathfrak{g}$-modules with atypicality equal to $\operatorname{def}(\mathfrak{g})$. If $\operatorname{atyp}(L(\lambda))=\operatorname{def}(\mathfrak{g})$, then $\operatorname{sdim} L(\lambda) \neq 0$. Therefore, by Corollary 6.5, $\mathcal{V}_{\mathfrak{e}}^{\text {rank }}(L(\lambda))=\mathfrak{e}_{\overline{1}}$.

\section{Appendix: Superalgebra data And sample Computations}

In this appendix we record various data for the simple Lie superalgebras of classical type and other related Lie superalgebras. We begin by quickly reviewing the structure of the simple classical Lie superalgebras; for more details on their definition and structure, including a precise description of $\mathfrak{g}_{\overline{1}}$ as a $\mathfrak{g}_{\overline{0}}$-supermodule, we refer the reader to Kac1, Chap. 2]. We next tabulate their cohomology rings. We then define the generic semisimple elements $x_{0}$ that one can use in both the Dadok-Kac and Luna-Richardson reduction arguments, and provide the data necessary to determine when $x_{0}$ is regular. We also provide explicit descriptions of the detecting subalgebras $\mathfrak{f}$ and $\mathfrak{e}$. Finally we give examples of both of the Dadok-Kac and Luna-Richardson computations in type $A$.

8.1. The Lie superalgebras of type A. Let $\mathfrak{g}=\mathfrak{g l}(m \mid n)$, the Lie superalgebra of $(m+n) \times(m+n)$ matrices over $\mathbb{C}$ with $\mathbb{Z}_{2}$-grading given by setting the degree of the matrix unit $E_{i, j}$ to be $\overline{0}$ if $1 \leq i, j \leq m$ or $m+1 \leq i, j \leq m+n$ and $\overline{1}$ otherwise. The bracket is the super-commutator bracket,

$$
[a, b]=a b-(-1)^{\bar{a} \bar{b}} b a,
$$


where $a, b \in \mathfrak{g}$ are assumed to be homogenous. The bracket of general elements is obtained by bilinearity. It is straightforward to verify that

$$
\mathfrak{g}_{\overline{0}} \cong \mathfrak{g l}(m) \oplus \mathfrak{g l}(n) \text { and } \mathfrak{g}_{\overline{1}} \cong V_{m} \otimes V_{n}^{*} \oplus V_{m}^{*} \otimes V_{n},
$$

where the first isomorphism is as Lie algebras, the second isomorphism is as $\mathfrak{g}_{0_{0}^{-}}$ modules, and where $V_{m}$ (resp. $\left.V_{n}\right)$ denotes the natural $\mathfrak{g l}(m)$-module (resp. $\mathfrak{g l}(n)$ module). For convenience, we shall say that $\mathfrak{g l}(m \mid n)$ is a Lie superalgebra of type $\widehat{A}(m-1, n-1)$.

Let $m, n \geq 1, m \neq n$ and let $\mathfrak{g}=\mathfrak{s l}(m \mid n)$ be the simple Lie superalgebra of type $A(m-1, n-1)$. That is, $\mathfrak{g}$ is the Lie subsuperalgebra of $\mathfrak{g l}(m \mid n)$ of all matrices with supertrace equal to zero. Then

$$
\mathfrak{g}_{\overline{0}} \cong \mathfrak{s l}(m) \oplus \mathfrak{s l}(n) \oplus \mathbb{C} \text { and } \mathfrak{g}_{\overline{1}} \cong V_{m} \otimes V_{n}^{*} \otimes \mathbb{C} \oplus V_{m}^{*} \otimes V_{n} \otimes \mathbb{C},
$$

where $\mathbb{C}$ denotes the one dimensional trivial Lie algebra and the trivial representation, respectively.

Let $m=n \geq 2$ and let $\mathfrak{g}=\mathfrak{p s r}(n \mid n)$ be the simple Lie superalgebra of type $A(n-1, n-1)$. That is, if $\widetilde{\mathfrak{g}}=\mathfrak{s l}(n \mid n)$ is the Lie subsuperalgebra of $\mathfrak{g l}(m \mid n)$ of all matrices with supertrace equal to zero, then $\mathfrak{g}$ is the quotient of $\widetilde{\mathfrak{g}}$ by the onedimensional ideal spanned by the identity matrix. Then one obtains

$$
\mathfrak{g}_{\overline{0}} \cong \mathfrak{s l}(n) \oplus \mathfrak{s l}(n) \text { and } \mathfrak{g}_{\overline{1}} \cong V_{n} \otimes V_{n}^{*} \oplus V_{n}^{*} \otimes V_{n} .
$$

8.2. The simple Lie superalgebra of type $\mathbf{P}$. Let $\mathfrak{g}$ be the simple Lie superalgebra of type $P(n-1)$ where $n \geq 3$, consisting of $2 n \times 2 n$ matrices of the form

$$
\left(\begin{array}{c|c}
A & B \\
\hline C & -A^{t}
\end{array}\right),
$$

where $A, B$ and $C$ are $n \times n$ matrices over $\mathbb{C}$ with $\operatorname{tr} A=0, B$ symmetric, and $C$ skew-symmetric. Then

$$
\mathfrak{g}_{\overline{0}} \cong \mathfrak{s} \mathfrak{l}(n) \text { and } \mathfrak{g}_{\overline{1}} \cong S^{2} V_{n} \oplus \Lambda^{2} V_{n}^{*},
$$

where $V_{n}$ is the natural $\mathfrak{s l}(n)$-module.

8.3. The Lie superalgebras of type $\mathbf{Q}$. Let $\mathfrak{g}=\mathfrak{q}(n)$ denote the Lie subsuperalgebra of $\mathfrak{g l}(n \mid n)$ consisting of $2 n \times 2 n$ matrices of the form

$$
\left(\begin{array}{l|l}
A & B \\
\hline B & A
\end{array}\right)
$$

where $A$ and $B$ are both $n \times n$ matrices over $\mathbb{C}$. It is again straightforward to verify that

$$
\mathfrak{g}_{\overline{0}} \cong \mathfrak{g l}(n) \text { and } \mathfrak{g}_{\overline{1}} \cong \mathfrak{g l}(n),
$$

where we mean $\mathfrak{g}_{\overline{1}}$ is the adjoint representation for $\mathfrak{g}_{\overline{0}}$. For convenience we shall say that $\mathfrak{q}(n)$ is a Lie superalgebra of type $\widehat{Q}(n-1)$.

Let $\mathfrak{g}$ denote the simple Lie superalgebra of type $Q(n-1)$, where $n \geq 3$. That is, $\mathfrak{g}$ is the Lie subsuperalgebra of $\mathfrak{q}(n)$ of all matrices of the form (8.3.1) such that $A$ and $B$ have trace zero. Then clearly

$$
\mathfrak{g}_{\overline{0}} \cong \mathfrak{s l}(n) \text { and } \mathfrak{g}_{\overline{1}} \cong \mathfrak{s l}(n) .
$$


8.4. The Lie superalgebras of type BCD. Let $\mathfrak{g}=\mathfrak{o s p}(m \mid n)$. That is, let $V$ denote a superspace with $\operatorname{dim} V_{\overline{0}}=m$ and $\operatorname{dim} V_{\overline{1}}=n$ and equipped with a fixed non-degenerate supersymmetric bilinear form $\beta$ such that with respect to this form $V_{\overline{0}}$ and $V_{\overline{1}}$ are orthogonal subspaces. In particular, note that the form is skew symmetric on $V_{\overline{1}}$, so necessarily $n$ is even. One defines $\mathfrak{g}$ to be the Lie subsuperalgebra of $\mathfrak{g l}(m \mid n)$ given by

$$
\mathfrak{o s p}(m \mid n)=\left\{x \in \mathfrak{g l}(V) \mid \beta(x(v), w)=-(-1)^{\bar{x}} \bar{v} \beta(v, x(w)) \text { for all } v, w \in V\right\} .
$$

Then

$$
\mathfrak{g}_{\overline{0}} \cong \mathfrak{s} \mathfrak{o}(m) \oplus \mathfrak{s p}(n) \text { and } \mathfrak{g}_{\overline{1}} \cong V_{m} \otimes V_{n},
$$

where $V_{m}$ (resp. $\left.V_{n}\right)$ is the natural representation for $\mathfrak{s o}(m)($ resp. $\mathfrak{s p}(n))$.

The classification types of the simple Lie superalgebras $\mathfrak{o s p}(m \mid n)$ are as follows: type $B(m, n)=\mathfrak{o s p}(2 m+1 \mid 2 n), m \geq 0, n>0 ;$ type $D(m, n)=\mathfrak{o s p}(2 m \mid 2 n)$, $m \geq 2, n>0$; type $C(n)=\mathfrak{o s p}(2 \mid 2 n-2), n \geq 2$.

8.5. The simple Lie superalgebra of type $D(2,1 ; \alpha)$. Let $\mathfrak{g}$ denote the simple Lie subsuperalgebra of type $D(2,1 ; \alpha)(\alpha \in \mathbb{C})$ as described in [Kac1]. Then

$$
\mathfrak{g}_{\overline{0}} \cong \mathfrak{s l}(2) \oplus \mathfrak{s l}(2) \oplus \mathfrak{s l}(2) \text { and } \mathfrak{g}_{\overline{1}} \cong V_{2} \otimes V_{2} \otimes V_{2},
$$

where $V_{2}$ denotes the natural $\mathfrak{s l}(2)$-module.

8.6. The simple Lie superalgebra of type $G(3)$. Let $\mathfrak{g}$ denote the simple Lie superalgebra of type $G(3)$. Then

$$
\mathfrak{g}_{\overline{0}} \cong \mathfrak{a} \oplus \mathfrak{s l}(2) \text { and } \mathfrak{g}_{\overline{1}} \cong W_{7} \otimes V_{2},
$$

where $\mathfrak{a}$ denotes the simple Lie algebra of type $G_{2}, W_{7}$ denotes the non-trivial $\mathfrak{a}$-module of minimal dimension and $V_{2}$ denotes the natural $\mathfrak{s l}(2)$-module.

8.7. The simple Lie superalgebra of type $F(4)$. Let $\mathfrak{g}$ denote the simple Lie superalgebra of type $F(4)$. Then

$$
\mathfrak{g}_{\overline{0}} \cong \mathfrak{a} \oplus \mathfrak{s l}(2) \text { and } \mathfrak{g}_{\overline{1}} \cong \operatorname{spin}_{7} \otimes V_{2},
$$

where $\mathfrak{a}$ denotes the simple Lie algebra of type $B_{3}, \operatorname{spin}_{7}$ denotes the $\mathfrak{a}$-module $\operatorname{spin}_{7}$ and $V_{2}$ denotes the natural $\mathfrak{s l}(2)$-module.

8.8. Cohomology rings. One of our main objects of interest is the relative cohomology ring $\mathrm{H}^{\bullet}\left(\mathfrak{g}, \mathfrak{g}_{0} ; \mathbb{C}\right)$, where $\mathfrak{g}$ is one of the classical Lie superalgebras described in the previous subsections. According to Theorem 2.6. $\mathrm{H}^{\bullet}\left(\mathfrak{g}, \mathfrak{g}_{0} ; \mathbb{C}\right) \cong S\left(\mathfrak{g}_{\overline{1}}^{*}\right)^{G_{\overline{0}}}$, and so we can exhibit these rings simply by referring to known calculations in invariant theory. They turn out to be polynomial rings in every case.

In Table 1 we list the Krull dimension of each relative cohomology ring, the degrees of its generators in the $\mathbb{Z}$-grading (recall that the $\mathbb{Z}_{2}$-grading is obtained by reducing the $\mathbb{Z}$-grading modulo 2 ), and a reference where the result can be found. Given the rich history of invariant theory, we make no claim in our references as to the original source of these calculations. By convention, set $r:=\min (m, n)$. 
TABLE 1. Cohomology rings for classical Lie superalgebras

\begin{tabular}{cccc}
$\mathfrak{g}$ & $\operatorname{dim} S\left(\mathfrak{g}_{1}^{*}\right)^{G_{\overline{0}}}$ & Degrees of Generators & Reference \\
\hline $\mathfrak{g l}(m \mid n)$ & $r$ & $2,4, \ldots, 2 r$ & [Fuk, Sec. 2.1.2] \\
$\mathfrak{s l}(m \mid n), m \neq n$ & $r$ & $2,4, \ldots, 2 r$ & Gru1] \\
$\mathfrak{p} \mathfrak{s}(n \mid n)$ & $n+1$ & $2,4, \ldots, 2 n-2, n, n$ & Section 8.11$]$ \\
$\mathfrak{o} \mathfrak{s}(2 m+1 \mid 2 n)$ & $r$ & $4,8, \ldots, 4 r$ & [Kac3, Tables II, III] \\
$\mathfrak{o s p}(2 m \mid 2 n), m>n$ & $n$ & $4,8, \ldots, 4 n$ & [Kac3, Tables II, III] \\
$\mathfrak{o s p}(2 m \mid 2 n), m \leq n$ & $m$ & $4,8, \ldots, 4 m-4,2 m$ & [Kac3, Tables II, III] \\
$P(n-1), n=2 l+1$ & $l+1$ & $4,8, \ldots, 4 l, n$ & [Gru2, Sch1] \\
$P(n-1), n=2 l$ & $l+1$ & $4,8, \ldots, 4 l-4, l, n$ & [Gru2, Sch1] \\
$\widehat{Q}(n-1)$ & $n$ & $1,2, \ldots, n$ & Wey] \\
$Q(n-1)$ & $n-1$ & $2,3, \ldots, n$ & Wey] \\
$D(2,1 ; \alpha)$ & 1 & 4 & [MW] \\
$G(3)$ & 1 & 4 & Kac3, Table IV] \\
$F(4)$ & 1 & 4 & Kac3, Table III]
\end{tabular}

8.9. Explicit detecting subalgebras. In this and the next two subsections we give explicit descriptions of the detecting subalgebras (cf. Section 4.1) for each classical Lie superalgebra $\mathfrak{g}$. Recall that we begin by defining a generic element $x_{0} \in \mathfrak{g}_{\overline{1}}$; this will be a certain linear combination of sums of positive and negative odd root vectors. Using the following theorem of Dadok and Kac [DK, Proposition $1.2]$, we will deduce that the $G_{\overline{0}}$-orbit of $x_{0}$ is closed; that is, $x_{0}$ is semisimple.

Theorem 8.1. Suppose $v_{\mu_{1}}, \ldots, v_{\mu_{s}}$ is a set of non-zero weight vectors in a $G$ module $V$ such that

(1) the weights $\mu_{1}, \ldots, \mu_{s}$ are distinct;

(2) 0 is an interior point of the convex hull of $\mu_{1}, \ldots, \mu_{s}$;

(3) $\mu_{i}-\mu_{j}$ is not a root if $i \neq j$.

Then the $G$ orbit of $v_{\mu_{1}}+\cdots+v_{\mu_{s}}$ is closed in $V$.

Furthermore, in all but two cases, the subspace $\mathfrak{e}_{x_{0}}$ is a Cartan subspace of $\mathfrak{g}_{\overline{1}}$, so the action of $G_{\overline{0}}$ on $\mathfrak{g}_{\overline{1}}$ is polar (cf. Section [3.3). One can then use the DadokKac theorem [DK] to compute the cohomology ring $S\left(\mathfrak{g}_{1}^{*}\right)^{G_{\overline{0}}}$ as in Section 3.3 An example of these computations is given in Section 8.10 .

In the remaining two cases, the action is stable, and we can apply the LunaRichardson theorem [LR] to compute $S\left(\mathfrak{g}_{1}^{*}\right)^{G_{\overline{0}}}$ as in Section 3.2. An example computation is carried out in Section 8.11

We begin with the definitions. Let $\mathfrak{g}$ be one of the classical Lie superalgebras discussed in Sections 8.18 .7 other than types $\widehat{Q}$ and $Q$. Let $\Omega$ be the set of odd positive roots defined in Table 2. (As before, we set $r=\min (m, n)$. Notation for the roots follows [Kac1.) Fix an element $x_{0}=\sum_{\alpha \in \Omega} d_{\alpha}\left(x_{\alpha}+x_{-\alpha}\right) \in \mathfrak{g}_{1}$, where $\left(d_{\alpha}\right)_{\alpha \in \Omega}$ is a vector of complex numbers which is not in the zero locus of the Jacobian $J$ of Table 5. (For $\mathfrak{p s l}(n \mid n)$ and $P(n-1)$ we assume the $d_{\alpha}$ are non-zero

\footnotetext{
${ }^{1}$ In [Kac3 Table II], for $S L_{n} \otimes S O_{m}, 1 \leq n<m, d$ should be $2 n$, not $2 m$, and for $S L_{n} \otimes S p_{m}$, $2 \leq n \leq m, n$ even, $d$ should be $n$, not $m$. Also, in [Kac3] Table III], for $S O_{n} \otimes S p_{m}, 2<n \leq m$, $n$ even, $d_{1}$ should be $n$, not $m$.
} 
with distinct squares.) The set $\Omega \cup-\Omega$ satisfies the conditions of Theorem 8.1 and so $x_{0}$ is semisimple.

TABLE 2. Sets $\Omega$ defining $x_{0}, \mathfrak{e}_{\overline{1}}$, and $\mathfrak{f}_{\overline{1}}$ for classical Lie superalgebras

\begin{tabular}{cc}
$\mathfrak{g}$ & $\Omega$ \\
\hline $\mathfrak{g l}(m \mid n)$ & $\left\{\epsilon_{i}-\epsilon_{m+i} \mid 1 \leq i \leq r\right\}$ \\
$\mathfrak{s l}(m \mid n), m \neq n$ & $\left\{\epsilon_{i}-\epsilon_{m+i} \mid 1 \leq i \leq r\right\}$ \\
$\mathfrak{p s l}(n \mid n)$ & $\left\{\epsilon_{i}-\epsilon_{n+i} \mid 1 \leq i \leq n\right\}$ \\
$\mathfrak{o s p}(2 m+1 \mid 2 n)$ & $\left\{\epsilon_{i}-\delta_{i} \mid 1 \leq i \leq r\right\}$ \\
$\mathfrak{o s p}(2 m \mid 2 n)$ & $\left\{\epsilon_{i}-\delta_{i} \mid 1 \leq i \leq r\right\}$ \\
$P(n-1), n$ even & $\left\{\epsilon_{i}+\epsilon_{n+1-i} \mid 1 \leq i \leq \frac{n}{2}\right\}$ \\
$P(n-1), n$ odd & $\left\{\epsilon_{i}+\epsilon_{n+1-i} \mid 1 \leq i \leq \frac{n+1}{2}\right\}^{2}$ \\
$D(2,1 ; \alpha)$ & $\left\{\epsilon_{1}+\epsilon_{2}+\epsilon_{3}\right\}$ \\
$G(3)$ & $\left\{\epsilon_{1}+\delta\right\}$ \\
$F(4)$ & $\left\{\frac{1}{2}\left(\epsilon_{1}+\epsilon_{2}+\epsilon_{3}+\epsilon_{4}\right)\right\}$
\end{tabular}

Define subspaces

$$
\begin{aligned}
& \mathfrak{e}_{\overline{1}}=\left\{\sum_{\alpha \in \Omega} c_{\alpha}\left(x_{\alpha}+x_{-\alpha}\right) \mid c_{\alpha} \in \mathbb{C}\right\} \\
& \mathfrak{f}_{\overline{1}}=\left\{\sum_{\alpha \in \Omega}\left(u_{\alpha} x_{\alpha}+v_{\alpha} x_{-\alpha}\right) \mid u_{\alpha}, v_{\alpha} \in \mathbb{C}\right\}
\end{aligned}
$$

of $\mathfrak{g}_{\overline{1}}$. Evidently $x_{0} \in \mathfrak{e}_{\overline{1}} \subset \mathfrak{f}_{\overline{1}}$.

Now assume we are in one of the cases $\widehat{Q}(n-1)$ or $Q(n-1)$. Recall from Section 8.3 that an element $x \in \mathfrak{g}_{1}$ is determined by an $n \times n$ matrix $B$ as in (8.3.1). When $B$ is diagonal (having trace zero in type $Q(n-1)$ ), $x$ lies in the 0 -root space. We take $x_{0}$ to be an element of $\mathfrak{g}_{\overline{1}}$ whose $B$ part is a diagonal matrix with $n$ distinct diagonal entries. From the classical theory of $\mathfrak{g l}(n)$ and $\mathfrak{s l}(n)$ one knows that $x_{0}$ is generic. We let $\mathfrak{e}_{\overline{1}}$ be the set of all $x \in \mathfrak{g}_{\overline{1}}$ whose $B$ matrix is diagonal (and trace zero in type $Q(n-1))$. Finally, we take $\mathfrak{f}_{\overline{1}}=\mathfrak{e}_{\overline{1}}$.

8.10. Dadok-Kac calculations. Except for the simple Lie superalgebras of types $A(n, n)$ and $P(n)$, one can check (see the representative example below) that

$$
\mathfrak{e}_{\overline{1}} \subseteq \mathfrak{e}_{x_{0}}:=\left\{y \in \mathfrak{g}_{\overline{1}} \mid\left[\mathfrak{g}_{\overline{0}}, y\right] \subseteq\left[\mathfrak{g}_{\overline{0}}, x_{0}\right]\right\}
$$

(recall (3.3.1)). Moreover, using Table 11, $\operatorname{dim} \mathfrak{e}_{\overline{1}}=\operatorname{dim} S\left(\mathfrak{g}_{1}^{*}\right)^{G_{\overline{0}}}$, and so it follows from the discussion in Section 3.3 that $\mathfrak{e}_{\overline{1}}=\mathfrak{e}_{x_{0}}$ is a Cartan subspace and the action of $G_{\overline{0}}$ on $\mathfrak{g}_{\overline{1}}$ is polar.

For example, consider the superalgebra $\mathfrak{g l}(m \mid n)$. Since $\mathfrak{g l}(m \mid n) \cong \mathfrak{g l}(n \mid m)$, one may assume $m \leq n$. Then $\Omega=\left\{\beta_{i}:=\epsilon_{i}-\epsilon_{m+i} \mid 1 \leq i \leq m\right\}$ and we write $x_{0}=$ $\sum_{i=1}^{m} d_{i}\left(x_{\beta_{i}}+x_{-\beta_{i}}\right)$ (with $d_{i} \neq 0$ for all $i$ and $d_{i}^{2} \neq d_{j}^{2}$ for $i \neq j$ ). Conditions (1)-(3) of Theorem 8.1 are immediate for the set of weights $\Omega \cup-\Omega$ appearing in $x_{0}$. To

\footnotetext{
${ }^{2}$ For $i_{0}:=(n+1) / 2$, the negative of the $\operatorname{root} \beta_{i_{0}}=\epsilon_{i_{0}}+\epsilon_{n+1-i_{0}}=2 \epsilon_{i_{0}}$ is not a root in $P(n-1)$, so terms involving $-\beta_{i_{0}}$ in the definitions of $x_{0}$ and $\mathfrak{f}_{\overline{1}}$ are to be ignored. For example, $x_{0}=\sum_{i=1}^{i_{0}-1} d_{i}\left(x_{\beta_{i}}+x_{-\beta_{i}}\right)+d_{i_{0}} x_{\beta_{i_{0}}}$.
} 
check 8.10.1) it suffices to verify that for every root vector $z=x_{\gamma} \in \mathfrak{g}_{0}$ or $z \in \mathfrak{h}$, and for every $y \in \mathfrak{e}_{\overline{1}}$, there exists $z^{\prime} \in \mathfrak{g}_{\overline{0}}$ such that

$$
[z, y]=\left[z^{\prime}, x_{0}\right]
$$

In fact, because of the symmetry of $y$ and $x_{0}$ with respect to positive and negative root vectors, we need only consider $\gamma \in \Phi_{\overline{0}}^{+}$. Write $y=\sum_{i=1}^{m} c_{i}\left(x_{\beta_{i}}+x_{-\beta_{i}}\right)$.

Suppose first that $\gamma=\epsilon_{j}-\epsilon_{k}$ with $1 \leq j<k \leq m$, so that $z=E_{j, k}$ (the standard matrix unit). Consider $z^{\prime}=a E_{j, k}+b E_{m+j, m+k}$ with $a, b \in \mathbb{C}$ to be determined. Then (8.10.2) becomes

$$
\left(\begin{array}{cc}
d_{k} & -d_{j} \\
-d_{j} & d_{k}
\end{array}\right)\left(\begin{array}{l}
a \\
b
\end{array}\right)=\left(\begin{array}{c}
c_{k} \\
-c_{j}
\end{array}\right),
$$

which has a solution since $d_{k}^{2}-d_{j}^{2} \neq 0$. A similar analysis applies to $\gamma=\epsilon_{m+j}-\epsilon_{m+k}$.

Next, if $\gamma=\epsilon_{m+j}-\epsilon_{k}$ with $1 \leq j \leq m$ and $2 m<k \leq m+n$, so that $z=E_{m+j, k}$, then $z^{\prime}=-\left(c_{j} / d_{j}\right) z$ satisfies (8.10.2). Also, if $\gamma=\epsilon_{j}-\epsilon_{k}$ with $2 m<j<k \leq m+n$, then $[z, y]=0$.

Finally, if $z=E_{j, j} \in \mathfrak{h}(1 \leq j \leq m+n)$, then taking $z^{\prime}=\left(c_{j} / d_{j}\right) z$ if $1 \leq j \leq m$, $z^{\prime}=\left(c_{j-m} / d_{j-m}\right) z$ if $m<j \leq 2 m$, and $z^{\prime}=0$ if $2 m<j \leq m+n$ satisfies (8.10.2).

For the corresponding simple Lie superalgebra $\mathfrak{s l}(m \mid n)$ with $m<n$, the same definitions of $\Omega, \mathfrak{e}_{1}$, and $x_{0}$ work, and the only changes to the proof involve the situation $z \in \mathfrak{h}$, due to the condition $\operatorname{str}(z)=\operatorname{str}\left(z^{\prime}\right)=0$. But we can take advantage of the fact that $\left[E_{k, k}, x_{0}\right]=0$ for $2 m<k \leq m+n$ to find, for any $z \in \mathfrak{h}$, a $z^{\prime} \in \mathfrak{h}$ satisfying (8.10.2). The remaining details are left to the reader.

However, for $\mathfrak{s l}(n \mid n)$ this argument breaks down, and in fact one can show that there is no Cartan subspace, so the action is not polar. A similar situation arises with the simple Lie superalgebra of type $P(n-1)$. In both cases the action is stable. If it were also polar, then the Cartan subspace would contain a generic element $x_{0}$. By maximality of orbit dimension, one sees that $\mathfrak{e}_{x_{0}}$ would equal the Cartan subspace. However, by a direct calculation we verified that in these two cases $\operatorname{dim} \mathfrak{e}_{x_{0}}=1<\operatorname{dim} S\left(\mathfrak{g}_{1}^{*}\right)^{G_{\overline{0}}}$, so the action cannot be polar. For these two Lie superalgebras we use the Luna-Richardson theory instead.

Returning to the general setup, write $H=G_{\overline{0}, x_{0}}$. Using [Sch1, Theorem 7] one sees that $x_{0}$ is regular, provided

$$
\operatorname{dim} H=\operatorname{dim} \mathfrak{g}_{\overline{0}}-\operatorname{dim} \mathfrak{g}_{\overline{1}}+\operatorname{dim} S\left(\mathfrak{g}_{\overline{1}}^{*}\right)^{G_{\overline{0}}} .
$$

In Table 3 we give the dimensions of $\mathfrak{g}_{\overline{0}}$ and $\mathfrak{g}_{\overline{1}}$ for the classical Lie superalgebras of Sections 8.1 8.7. Table 4 gives the structure of $H$ and its dimension. (In the table, $T$ denotes the torus $\mathbb{C}^{\times}$.) In particular, we observe that in every case, $H$ is connected. Using these data, along with the dimensions of the cohomology rings from Table 1, one can check that $x_{0}$ is regular (and thus the action of $G_{\overline{0}}$ on $\mathfrak{g}_{\overline{1}}$ is stable) in all but one case. Table 5 lists which cases are polar or stable. In the polar cases, we give the structure of the Dadok-Kac group $W=\operatorname{Norm}_{G_{\overline{0}}}\left(\mathfrak{e}_{\overline{1}}\right) / \operatorname{Stab}_{G_{\overline{0}}}\left(\mathfrak{e}_{\overline{1}}\right)$ along with its associated Jacobian $J$ (cf. Section 3.3). The notation $\left(\mathbb{Z}_{4}^{r}\right)_{e}$ means the subgroup of $r$-tuples of elements of $\mathbb{Z}_{4}=\langle\sqrt{-1}\rangle$ having an even number of entries $\pm \sqrt{-1}$. In each case the action of $W$ on coordinates on $\mathfrak{e}_{\overline{1}} \simeq \mathbb{C}^{r}$ is the obvious one, from which the description of the rings of invariant polynomials in Table 1 can be verified. 
TABLE 3. Dimensions of classical Lie superalgebras

\begin{tabular}{ccc}
$\mathfrak{g}$ & $\operatorname{dim} \mathfrak{g}_{\overline{0}}$ & $\operatorname{dim} \mathfrak{g}_{\overline{1}}$ \\
\hline $\mathfrak{g l}(m \mid n)$ & $m^{2}+n^{2}$ & $2 m n$ \\
$\mathfrak{s l}(m \mid n), m \neq n$ & $m^{2}+n^{2}-1$ & $2 m n$ \\
$\mathfrak{p} \mathfrak{s}(n \mid n)$ & $2 n^{2}-2$ & $2 n^{2}$ \\
$\mathfrak{o s p}(2 m+1 \mid 2 n)$ & $2 m^{2}+m+2 n^{2}+n$ & $(2 m+1)(2 n)$ \\
$\mathfrak{o s p}(2 m \mid 2 n)$ & $2 m^{2}-m+2 n^{2}+n$ & $4 m n$ \\
$P(n-1)$ & $n^{2}-1$ & $n^{2}$ \\
$\widehat{Q}(n-1)$ & $n^{2}$ & $n^{2}$ \\
$Q(n-1)$ & $n^{2}-1$ & $n^{2}-1$ \\
$D(2,1 ; \alpha)$ & 9 & 8 \\
$G(3)$ & 17 & 14 \\
$F(4)$ & 24 & 16
\end{tabular}

TABle 4. Centralizer of $x_{0}$

\begin{tabular}{ccc}
$\mathfrak{g}$ & $H$ & $\operatorname{dim} H$ \\
\hline $\mathfrak{g l}(m \mid n)$ & $T^{r} \times G L_{|n-m|}$ & $r+(n-m)^{2}$ \\
$\mathfrak{s l}(m \mid n), m \neq n$ & $T^{r} \times S L_{|n-m|}$ & $r+(n-m)^{2}-1$ \\
$\mathfrak{p} \mathfrak{l}(n \mid n)$ & $T^{n-1}$ & $n-1$ \\
$\mathfrak{o s p}(2 m+1 \mid 2 n), m \geq n$ & $T^{r} \times S O_{2(m-n)+1}$ & $r+2(m-n)^{2}+m-n$ \\
$\mathfrak{o} \mathfrak{s p}(2 m+1 \mid 2 n), m<n$ & $T^{r} \times S p_{2(n-m)}$ & $r+2(n-m)^{2}+n-m$ \\
$\mathfrak{o} \mathfrak{s p}(2 m \mid 2 n), m>n$ & $T^{r} \times S O_{2(m-n)}$ & $r+2(n-m)^{2}+(n-m)$ \\
$\mathfrak{o} \mathfrak{s}(2 m \mid 2 n), m \leq n$ & $T^{r} \times S p_{2(n-m)}$ & $r+2(n-m)^{2}+(n-m)$ \\
$P(n-1)$ & $T^{\lfloor n / 2\rfloor}$ & $\lfloor n / 2\rfloor$ \\
$\widehat{Q}(n-1)$ & $T^{n}$ & $n$ \\
$Q(n-1)$ & $T^{n-1}$ & $n-1$ \\
$D(2,1 ; \alpha)$ & $T^{2}$ & 2 \\
$G(3)$ & $S L_{2} \times T$ & 4 \\
$F(4)$ & $S L_{3} \times T$ & 9
\end{tabular}

8.11. Luna-Richardson calculations. Here we apply the Luna-Richardson theory to $\mathfrak{g}=\mathfrak{p s r}(n \mid n)$, to compute $S\left(\mathfrak{g}_{\overline{1}}^{*}\right)^{G_{\overline{0}}}$. We have $G_{\overline{0}} \simeq S L(n) \times S L(n)$ and $\mathfrak{g}_{\overline{1}} \simeq M_{n}(\mathbb{C}) \oplus M_{n}(\mathbb{C})$, with action $(A, B) \cdot(X, Y)=\left(A X B^{-1}, B Y A^{-1}\right)(A, B \in$ $\left.S L(n), X, Y \in M_{n}(\mathbb{C})\right)$. Let $\Omega=\left\{\beta_{1}, \ldots, \beta_{n}\right\} \subset \Phi_{\overline{1}}^{+}$and $x_{0} \in \mathfrak{g}_{\overline{1}}$ be as defined in the previous subsection for $\mathfrak{g l}(m \mid n)$ with $m=n$. Then $H:=G_{\overline{0}}, x_{0}=\left\{(D, D) \in G_{\overline{0}} \mid\right.$ $D$ is diagonal $\}$ and $\mathfrak{f}_{\overline{1}}:=\mathfrak{g}_{\overline{1}}^{H}=\left\{\sum_{j=1}^{n} u_{j} x_{\beta_{j}}+v_{j} x_{-\beta_{j}} \mid u_{j}, v_{j} \in \mathbb{C}\right\}$. An element $(A, B)$ of the normalizer $N:=N_{G_{\overline{0}}}(H)$ is determined by a permutation $\sigma \in \Sigma_{n}$ and 
TABLE 5. Polar and stable classical Lie superalgebras

\begin{tabular}{ccccc}
$\mathfrak{g}$ & Polar & Stable & $W$ & $J$ \\
\hline $\mathfrak{g l}(m \mid n)$ & Yes & Yes & $\Sigma_{r} \ltimes \mathbb{Z}_{2}^{r}$ & $x_{1} \ldots x_{r} \prod_{i<j}\left(x_{i}^{2}-x_{j}^{2}\right)$ \\
$\mathfrak{s l}(m \mid n), m \neq n$ & Yes & Yes & $\Sigma_{r} \ltimes \mathbb{Z}_{2}^{r}$ & $x_{1} \ldots x_{r} \prod_{i<j}\left(x_{i}^{2}-x_{j}^{2}\right)$ \\
$\mathfrak{p s l}(n \mid n)$ & No & Yes & - & - \\
$\mathfrak{o s p}(2 m+1 \mid 2 n), m \geq n$ & Yes & Yes & $\Sigma_{r} \ltimes \mathbb{Z}_{4}^{r}$ & $x_{1} \ldots x_{r} \prod_{i<j}\left(x_{i}^{4}-x_{j}^{4}\right)$ \\
$\mathfrak{o s p}(2 m+1 \mid 2 n), m<n$ & Yes & No & $\Sigma_{r} \ltimes \mathbb{Z}_{4}^{r}$ & $x_{1} \ldots x_{r} \prod_{i<j}\left(x_{i}^{4}-x_{j}^{4}\right)$ \\
$\mathfrak{o s p}(2 m \mid 2 n), m>n$ & Yes & Yes & $\Sigma_{r} \ltimes \mathbb{Z}_{4}^{r}$ & $x_{1} \ldots x_{r} \prod_{i<j}\left(x_{i}^{4}-x_{j}^{4}\right)$ \\
$\mathfrak{o s p}(2 m \mid 2 n), m \leq n$ & Yes & Yes & $\Sigma_{r} \ltimes\left(\mathbb{Z}_{4}^{r}\right)_{e}$ & $x_{1}^{2} \ldots x_{r}^{2} \prod_{i<j}\left(x_{i}^{4}-x_{j}^{4}\right)$ \\
$P(n-1)$ & No & Yes & - & - \\
$\widehat{Q}(n-1)$ & Yes & Yes & $\Sigma_{n}$ & $\prod_{i<j}\left(x_{i}-x_{j}\right)$ \\
$Q(n-1)$ & Yes & Yes & $\Sigma_{n}$ & $\prod_{i<j}\left(x_{i}-x_{j}\right)$ \\
$D(2,1 ; \alpha)$ & Yes & Yes & $Z_{4}$ & $x_{1}^{3}$ \\
$G(3)$ & Yes & Yes & $Z_{4}$ & $x_{1}^{3}$ \\
$F(4)$ & Yes & Yes & $Z_{4}$ & $x_{1}^{3}$
\end{tabular}

scalars $a_{j}, b_{j} \in \mathbb{C}^{\times}$with $\prod a_{j}=\prod b_{j}=\operatorname{sgn}(\sigma)$, where $A_{j, \sigma(j)}=a_{j}, B_{j, \sigma(j)}=b_{j}$ for $1 \leq j \leq n$, and all other entries of $A$ and $B$ are 0 . Then $(A, B)$ acts on a pair $(u, v) \in\left(\mathbb{C}^{n}\right)^{2}$ parametrizing an element of $\mathfrak{f}_{\overline{1}}$ by sending it to $\left(u^{\prime}, v^{\prime}\right)$, where $u_{j}^{\prime}=a_{j} b_{j}^{-1} u_{\sigma(j)}, v_{j}^{\prime}=a_{j}^{-1} b_{j} v_{\sigma(j)}$.

Abusing notation and viewing $u_{j}, v_{j}$ as coordinate functions on $\mathfrak{f}_{\overline{1}}$, we see that

$$
S\left(\mathfrak{f}_{\overline{1}}^{*}\right)^{N} \simeq \mathbb{C}\left[f_{2}, \ldots, f_{2 n-2}, g_{n}, h_{n}\right],
$$

where $f_{2}, \ldots, f_{2 n}$ are the elementary symmetric polynomials in $u_{1} v_{1}, \ldots, u_{n} v_{n}$, $g_{n}:=u_{1} \ldots u_{n}$, and $h_{n}:=v_{1} \ldots v_{n}$. Note that $f_{2 n}=g_{n} h_{n}$ so that $f_{2 n}$ is redundant; however, this is the only relation. We conclude, by the Luna-Richardson theorem, that $S\left(\mathfrak{g}_{1}^{*}\right)^{G_{\overline{0}}}$ is a polynomial algebra in $n+1$ generators in degrees $2,4, \ldots, 2 n-2 ; n, n$. (This result is apparently known to the experts in classical invariant theory Sch2, but we could not find it in the literature. Gruson Gru2 has an incorrect description of $S\left(\mathfrak{g}_{1}^{*}\right)^{G_{\overline{0}}}$. It is based on her earlier computation Gru1 in the case $m \neq n$, but does not seem to take into account the subtleties of the supertrace zero condition when $m=n$.)

A similar analysis can be carried out for the simple Lie superalgebra of type $P(n-1)$, as has been done by Gruson [Gru2]. The degrees of the generators of the polynomial invariants on $\mathfrak{f}_{\overline{1}}$ are given in Table 1 Alternately, the fact that $S\left(\mathfrak{g}_{\overline{1}}^{*}\right)^{G_{\overline{0}}}$ is a polynomial algebra can be deduced from [Sch1, Table 1a, lines 16-17], and the degrees of its generators can be determined using [Sch1, Table 1b] by omitting those generators having positive degree on $\varphi_{1}$ or $\varphi_{1}^{*}$.

\section{REFERENCES}

[AM] M. F. Atiyah and I. G. Macdonald, Introduction to commutative algebra, Addison-Wesley Publishing Co., Reading, Mass.-London-Don Mills, Ont., 1969. MR0242802 (39:4129) 
[Bal] P. Balmer, The spectrum of prime ideals in tensor triangulated categories, J. Reine Angew. Math. 588 (2005), 149-168. MR2196732 (2007b:18012)

[Ben] D. J. Benson, Representations and cohomology. II, second ed., Cambridge Studies in Advanced Mathematics, vol. 31, Cambridge University Press, Cambridge, 1998. MR 1634407 (99f:20001b)

[Bag] I. Bagci, Cohomology and support varieties for Lie superalgebras, Ph.D. Thesis, University of Georgia, 2009.

[BaKN] I. Bagci, J. R. Kujawa, and D. K. Nakano, Cohomology and support varieties for Lie superalgebras of type $W(n)$, Int. Math. Res. Notices, Vol. 2008, article ID rnn115 (2008). MR2448087

[BKN] B. D. Boe, J. R. Kujawa, and D. K. Nakano, Cohomology and support varieties for Lie superalgebras II, Proc. London Math. Soc. 98 (2009), 19-44. MR2472160

[Bru1] J. Brundan, Kazhdan-Lusztig polynomials and character formulae for the Lie superalgebra $\mathfrak{g l}(m \mid n)$, J. Amer. Math. Soc. 16 (2003), no. 1, 185-231 (electronic). MR.1937204 (2003k:17007)

[Bru2] Kazhdan-Lusztig polynomials and character formulae for the Lie superalgebra $\mathfrak{q}(n)$, Adv. Math. 182 (2004), no. 1, 28-77. MR2028496 (2004m:17018)

[Car] J. F. Carlson, The variety of an indecomposable module is connected, Invent. Math. $\mathbf{7 7}$ (1984), no. 2, 291-299. MR752822 (86b:20009)

[CWZ] S.-J. Cheng, W. Wang, and R. B. Zhang, A Fock space approach to representation theory of osp $(2 \mid 2 n)$, Transform. Groups. 12 (2007), 209-225. MR2323682 (2008b:17007)

[CPS] E. Cline, B. Parshall, and L. Scott, Finite-dimensional algebras and highest weight categories, J. Reine Angew. Math. 391 (1988), 85-99. MR961165 (90d:18005)

[DK] J. Dadok and V. G. Kac, Polar representations, J. Algebra 92 (1985), no. 2, 504-524. MR778464 (86e:14023)

[DS] M. Duflo and V. Serganova, On associated variety for Lie superalgebras, arXiv:math.RT/0507198, 2005.

[EH] K. Erdmann and M. Holloway, Rank varieties and projectivity for a class of local algebras, Math. Z. 247 (2004), no. 3, 441-460. MR.2114422 (2006c:16022)

[Far] R. Farnsteiner, Tameness and complexity of finite group schemes, Bull. London Math. Soc. 39 (2007), no. 1, 63-70. MR.2303520 (2007k:16032)

[FP1] E. M. Friedlander and B. J. Parshall, Geometry of p-unipotent Lie algebras, J. Algebra 109 (1987), no. 1, 25-45. MR898334 (89a:17017)

[FP2] E. M. Friedlander and J. Pevtsova, Representation-theoretic support spaces for finite group schemes, Amer. J. Math. 127 (2005), no. 2, 379-420. MR2130619 (2005k:14096)

[FL] D. B. Fuch and D. A. Ley̆tes, Cohomology of Lie superalgebras, C. R. Acad. Bulgare Sci. 37 (1984), no. 12, 1595-1596. MR826386 (87e:17022)

[Fuk] D. B. Fuks, Cohomology of infinite-dimensional Lie algebras, Contemporary Soviet Mathematics, Consultants Bureau, New York, 1986, Translated from the Russian by A. B. Sosinski1. MR 874337 (88b:17001)

[GJ] O. Gabber and A. Joseph, Towards the Kazhdan-Lusztig conjecture, Ann. Sci. École Norm. Sup. (4) 14 (1981), no. 3, 261-302. MR644519 (83e:17009)

[GW] R. Goodman and N. R. Wallach, Representations and invariants of the classical groups, Encyclopedia of Mathematics and its Applications, vol. 68, Cambridge University Press, Cambridge, 1998. MR 1606831 (99b:20073)

[Gru1] C. Gruson, Finitude de l'homologie de certains modules de dimension finie sur une super algèbre de Lie, Ann. Inst. Fourier (Grenoble) 47 (1997), no. 2, 531-553. MR.1450424 (98b:17024)

[Gru2] , Sur la cohomologie des super algèbres de Lie étranges, Transform. Groups 5 (2000), no. 1, 73-84. MR1745712 (2001c:17036)

[Hoc] G. Hochschild, Relative homological algebra, Trans. Amer. Math. Soc. 82 (1956), 246-269. MR0080654 (18:278a)

[Jan] J. C. Jantzen, Representations of algebraic groups, second ed., Mathematical Surveys and Monographs, vol. 107, American Mathematical Society, Providence, RI, 2003. MR.2015057 (2004h:20061)

[Kac1] V. G. Kac, Lie superalgebras, Advances in Math. 26 (1977), no. 1, 8-96. MR0486011 $(58: 5803)$ 
[Kac2] _ Representations of classical Lie superalgebras, Differential geometrical methods in mathematical physics, II (Proc. Conf., Univ. Bonn, Bonn, 1977), Lecture Notes in Math., vol. 676, Springer, Berlin, 1978, pp. 597-626. MR519631 (80f:17006)

[Kac3] _ Some remarks on nilpotent orbits, J. Algebra 64 (1980), no. 1, 190-213. MR.575790 (81i:17005)

[KW] V. G. Kac and M. Wakimoto, Integrable highest weight modules over affine superalgebras and number theory, Lie theory and geometry, Progr. Math., vol. 123, Birkhäuser Boston, Boston, MA, 1994, pp. 415-456. MR1327543 (96j:11056)

[KWe] V. G. Kac and B. Weisfeiler, Irreducible representations of Lie p-algebras, Funct. Anal. Appl. 5 (1971), 28-36. MR0285575 (44:2793)

[Kum] S. Kumar, Kac-Moody groups, their flag varieties and representation theory, Prog. Math., vol. 204, Birkhäuser Boston, Boston, MA, 2002. MR1923198 (2003k:22022)

[LR] D. Luna and R. W. Richardson, A generalization of the Chevalley restriction theorem, Duke Math. J. 46 (1979), no. 3, 487-496. MR.544240 (80k:14049)

[MW] D. A. Meyer and N. R. Wallach, Invariants for multiple qubits: The case of 3 qubits, Mathematics of quantum computation, Comput. Math. Ser., Chapman \& Hall/CRC, Boca Raton, FL, 2002, pp. 77-97. MR2007943 (2004h:81034)

$[\mathrm{MVdJ}]$ E. M. Moens and J. Van der Jeugt, A character formula for atypical critical $\mathfrak{g l}(m \mid n)$ representations labelled by composite partitions, J. Phys. A 37 (2004), no. 50, 1201912039. MR 2106623 (2005j:17008)

[Pan] D. I. Panyushev, On covariants of reductive algebraic groups, Indag. Math. (N.S.) 13 (2002), no. 1, 125-129. MR2014979 (2004i:13006)

[Pop] V. L. Popov, Stability of the action of an algebraic group on an algebraic variety, Izv. Akad. Nauk SSSR Ser. Mat. 36 (1972), 371-385. MR0301028 (46:188)

[PV] V. L. Popov and È. B. Vinberg, Invariant theory, Algebraic geometry. IV (A. N. Parshin and I. R. Shafarevich, eds.), Encylopaedia of Mathematical Sciences, vol. 55, SpringerVerlag, Berlin, 1994, pp. 123-278. MR.1309681 (95g:14002)

[Pre] A. A. Premet, Irreducible representations of Lie algebras of reductive groups and the KacWeisfeiler conjecture, Invent. Math. 121 (1995), no. 1, 79-117. MR1345285 (96g:17007)

[PS] I. Penkov and V. Serganova, Characters of finite-dimensional irreducible $\mathfrak{q}(n)$-modules, Lett. Math. Phys. 40 (1997), no. 2, 147-158. MR1463616 (98i:17011)

[Sch1] G. W. Schwarz, Representations of simple Lie groups with regular rings of invariants, Invent. Math. 49 (1978), no. 2, 167-191. MR.511189 (80m:14032)

[Sch2] , 2006, private e-mail communication.

[Ser1] V. Serganova, Kazhdan-Lusztig polynomials and character formula for the Lie superalgebra $\mathfrak{g l}(m \mid n)$, Selecta Math. (N.S.) 2 (1996), no. 4, 607-651. MR.1443186 (98f:17007)

[Ser2] _ On representations of the Lie superalgebra $p(n)$, J. Algebra 258 (2002), no. 2, 615-630. MR1943937 (2003i:17011)

[Ser3] _ A reduction method for atypical representations of classical Lie superalgebras, Adv. Math. 180 (2003), no. 1, 248-274. MR2019224(2004k:17015)

[Ser4] - On representations of Cartan type Lie superalgebras, Amer. Math. Soc. Transl. 213 (2005), no. 2, 223-239. MR2140724 (2006c:17011)

[Ser5] B Blocks in the category of finite-dimensional representations of $g l(m, n)$, preprint.

[Sha] I. R. Shafarevich, Basic algebraic geometry. 1, Varieties in projective space, second ed., Translated from the 1988 Russian edition and with notes by Miles Reid, Springer-Verlag, Berlin, 1994. MR1328833 (95m:14001)

[SS] N. Snashall and Ø. Solberg, Support varieties and Hochschild cohomology rings, Proc. London Math. Soc. (3) 88 (2004), no. 3, 705-732. MR2044054 (2005a:16014)

[TY] P. Tauvel and R. W. T. Yu, Lie algebras and algebraic groups, Springer Monographs in Mathematics, Springer-Verlag, Berlin, 2005. MR,2146652 (2006c:17001)

[VdJ] J. Van der Jeugt, Character formulae for the Lie superalgebra $C(n)$, Comm. Algebra 19 (1991), no. 1, 199-222. MR.1092559 (92b:17050)

[VdJZ] J. Van der Jeugt and R. B. Zhang, Characters and composition factor multiplicities for the Lie superalgebra $\mathfrak{g l}(m \mid n)$, Lett. Math. Phys. 47 (1999), no. 1, 49-61. MR1669394 (2000a:17008)

[WZ1] W. Wang and L. Zhao, Representations of Lie superalgebras in prime characteristic I, Proc. Lond. Math. Soc. (3) 99 (2009), no. 1, 145-167. MR2520353 
[WZ2] _ Representations of Lie superalgebras in prime characteristic II: The queer series, arXiv:0902.2758, 2009.

[Wey] H. Weyl, The classical groups. Their invariants and representations, Princeton University Press, Princeton, N.J., 1939.

Department of Mathematics, University of Georgia, Athens, Georgia 30602

E-mail address: brian@math.uga.edu

Department of Mathematics, University of Oklahoma, Norman, Oklahoma 73019

E-mail address: kujawa@math.ou.edu

Department of Mathematics, University of Georgia, Athens, Georgia 30602

E-mail address: nakano@math.uga.edu 